\title{
CHORDAL KOMATU-LOEWNER EQUATION AND BROWNIAN MOTION WITH DARNING IN MULTIPLY CONNECTED DOMAINS
}

\author{
ZHEN-QING CHEN, MASATOSHI FUKUSHIMA, AND STEFFEN ROHDE
}

\begin{abstract}
Let $D=\mathbb{H} \backslash \bigcup_{k=1}^{N} C_{k}$ be a standard slit domain where $\mathbb{H}$ is the upper half-plane and $C_{k}, 1 \leq k \leq N$, are mutually disjoint horizontal line segments in $\mathbb{H}$. Given a Jordan arc $\gamma \subset D$ starting at $\partial \mathbb{H}$, let $g_{t}$ be the unique conformal map from $D \backslash \gamma[0, t]$ onto a standard slit domain $D_{t}$ satisfying the hydrodynamic normalization. We prove that $g_{t}$ satisfies an ODE with the kernel on its right-hand side being the complex Poisson kernel of the Brownian motion with darning (BMD) for $D_{t}$, generalizing the chordal Loewner equation for the simply connected domain $D=\mathbb{H}$. Such a generalization has been obtained by Y. Komatu in the case of circularly slit annuli and by R. O. Bauer and R. M. Friedrich in the present chordal case, but only in the sense of the left derivative in $t$. We establish the differentiability of $g_{t}$ in $t$ to make the equation a genuine ODE. To this end, we first derive the continuity of $g_{t}(z)$ in $t$ with a certain uniformity in $z$ from a probabilistic expression of $\Im g_{t}(z)$ in terms of the BMD for $D$, which is then combined with a Lipschitz continuity of the complex Poisson kernel under the perturbation of standard slit domains to get the desired differentiability.
\end{abstract}

\section{INTRODUCTION}

A domain of the form $D=\mathbb{H} \backslash \bigcup_{k=1}^{N} C_{k}$ is called a standard slit domain, where $\mathbb{H}$ is the upper half-plane and $\left\{C_{k}\right\}$ are mutually disjoint horizontal line segments. We fix a standard slit domain $D$ and consider a Jordan arc

$$
\gamma:\left[0, t_{\gamma}\right] \rightarrow \bar{D}, \quad \gamma(0) \in \partial \mathbb{H}, \quad \gamma\left(0, t_{\gamma}\right] \subset D .
$$

For each $t \in\left[0, t_{\gamma}\right]$, let $g_{t}$ be the unique conformal map from $D \backslash \gamma(0, t]$ onto a standard slit domain $D_{t}=\mathbb{H} \backslash \bigcup_{k=1}^{N} C_{k, t}$ satisfying

$$
g_{t}(z)=z+\frac{a_{t}}{z}+o(1 /|z|), \quad z \rightarrow \infty,
$$

for some constant $a_{t}$. It can be shown (and we will give a self-contained proof in $\S 8)$ that $t \mapsto a_{t}$ is a real-valued strictly increasing continuous function with $a_{0}=0$

Received by the editors March 3, 2013 and, in revised form, April 7, 2014.

2010 Mathematics Subject Classification. Primary 60H30; Secondary 30C20.

Key words and phrases. Komatu-Loewner equation, Brownian motion with darning, harmonic function with zero period, Green function, complex Poisson kernel, multiply connected domain.

The first author's research was partially supported by NSF Grant DMS-1206276 and NNSFC Grant 11128101.

The second author's research was supported by Grant-in-Aid for Scientific Research of MEXT No. 22540125.

The third author's research was partially supported by NSF Grant DMS-1068105. 
so that the arc $\gamma$ can be reparametrized in a way that $a_{t}=2 t, 0 \leq t \leq t_{\gamma}$. Define

$$
\xi(t)=g_{t}(\gamma(t))(\in \partial \mathbb{H}), \quad 0 \leq t \leq t_{\gamma} .
$$

In [3], R. O. Bauer and R. M. Friedrich have derived under the above reparametrization of $\gamma$ a chordal Komatu-Loewner equation

$$
\frac{\partial^{-} g_{t}(z)}{\partial t}=-2 \pi \Psi_{t}\left(g_{t}(z), \xi(t)\right), \quad g_{0}(z)=z, \quad 0<t \leq t_{\gamma}
$$

where $\frac{\partial^{-} g_{t}(z)}{\partial t}$ denotes the left partial derivative in $t$. This is an extension of the Komatu-Loewner equation obtained first by Y. Komatu [12] for circularly slit annuli and later by Bauer-Friedrich [2] for circularly slit disks with an improved expression of the right-hand side. The kernel $\Psi_{t}(z, \zeta), z \in D_{t}, \zeta \in \partial \mathbb{H}$, appearing in (1.4) is an analytic function of $z \in D_{t}$ subjected to the normalization

$$
\lim _{z \in D_{t}, z \rightarrow \infty} \Psi_{t}(z, \zeta)=0 .
$$

However, the differentiability of $g_{t}(z)$ in $t$ has been established neither in the circularly slit cases $([12,2])$ nor in the chordal case (3]). We have three goals in the present paper, and a primary one of them is to prove in Theorem 9.9 this differentiability making (1.4) a genuine ordinary differential equation. While several results of this paper could be proved by methods of classical complex analysis, we will emphasize a more probabilistic approach.

The first goal is to present an alternative derivation of the Komatu-Loewner equation (1.4) by showing that the imaginary part $K_{t}^{*}(z, \zeta)$ of the kernel $\Psi_{t}(z, \zeta)$ is just the Poisson kernel of the Brownian motion with darning (abbreviated as BMD) for the standard slit domain $D_{t}$. In Chapter 7 of the book [5] by the first and second authors, the notion of BMD is introduced and its basic properties are studied. Roughly speaking, the BMD for a standard slit domain $D=\mathbb{H} \backslash$ $\bigcup_{k=1}^{N} C_{k}$ is a diffusion process on a state space $D^{*}=D \cup\left\{c_{1}^{*}, \cdots, c_{N}^{*}\right\}$ obtained from the absorbing Brownian motion (abbreviated as ABM) on $\mathbb{H}$ by regarding (or "shorting") each "hole" $C_{k}$ into one single point $c_{k}^{*}$. The BMD on $D^{*}$ is symmetric with respect to the measure $m^{*}$ that extends the Lebesgue measure $m$ on $D$ to $D^{*}$ by setting $m^{*}\left(\left\{c_{k}^{*}\right\}\right)=0,1 \leq k \leq N$. We are motivated by a paper [14] of G. Lawler in which it was claimed that $K^{*}(z, \zeta)$ is the Poisson kernel of the excursion reflected Brownian motion (abbreviated as ERBM) introduced there in a rather descriptive way using excursions. When the number of the slits is one, the ERBM was described more explicitly by S. Drenning [7] and, by that, the ERBM can be identified with our BMD as will be seen in $\S 2$. The paper [7] investigates a KomatuLoewner type equation for standard slit domains with the goal to confirm Lawler's claim that $K^{*}$ is the Poisson kernel of ERBM. The method in [7] is quite different from ours and particularly contains a comparison of $a_{t}$ with its counterpart in the simply connected domain $\mathbb{H}$.

The BMD is known ([5]) to be the unique $m^{*}$-symmetric diffusion extension of the ABM on $D$ to $D^{*}$ that admits no killing on $\left\{c_{k}^{*}, 1 \leq k \leq N\right\}$. In the next two sections of this paper, we present a self-contained short exposition of BMD and its basic properties that will be used later in the paper. In $\$ 2$ we give a simple and direct construction of BMD as well as its uniqueness proof by observing that the associated Dirichlet form on $L^{2}\left(D^{*} ; m^{*}\right)$ is regular due to a nice property (2.1) of the two-dimensional Brownian motion. We show in 93 that the $L^{2}$-generator of $\mathrm{BMD}$ is characterized by the zero flux property at each $c_{k}^{*}$. In 93,3 , we then derive 
from this zero flux condition the zero period property around $c_{k}^{*}$ for any function that is BMD-harmonic in a neighborhood of $c_{k}^{*}$. This zero period property readily yields in $\$ 5$ explicit expressions of the Green function and the Poisson kernel of $\mathrm{BMD}$ in terms of the periods of the harmonic basis.

It was proved in [3] that $a_{t}$ is strictly increasing, based on a certain extremal property of $g_{t}$. In $\$ 6$, we shall give a different proof, establish left-continuity, and by combining it with the above-mentioned explicit expression of the BMD-Poisson kernel and a general formula of the period of a harmonic function presented in 4 , we shall derive a chordal Komatu-Loewner equation formulated in terms of the left derivative in $a_{t}$.

The second goal of this paper is to present in $\$ 7$ a probabilistic representation of the conformal map $g_{t}$ and use it to derive in $\S 8$ the continuity of $g_{t}(z)$ in $t$ with uniformity in $z$ on each compact region including the boundary $\partial_{p} C_{k}$ of the set $\mathbb{H} \backslash C_{k}$ in the path distance topology, thereby obtaining the continuity of $a_{t}, D_{t}$ as well as $\xi(t)$. To this end, a construction of a BMD-harmonic function $v^{*}$ on a general $(N+1)$-connected domain $D=\mathbb{H} \backslash \bigcup_{i=1}^{N} A_{i}$ with a compact $\mathbb{H}$-hull $F$ removed will be carried out in Appendix 1 using a hitting probability of BMD by following the method of Lawler [14, §5], where ERBM was used in place of BMD. The analytic map $f$ with imaginary part $v^{*}$ will then be shown to be a conformal mapping from $D \backslash F$ onto a standard slit domain by invoking a degree theorem, which is presented in Appendix 2. Thus the imaginary part $\Im g_{t}(z)$ of $g_{t}(z)$ admits a probabilistic representation in terms of BMD on $D^{*}$ and ABM on $\mathbb{H}$. The continuity of $g_{t}(z)$ in $t$ then follows from the stochastic continuity of the first hitting time of the set $\gamma[0, t]$ by BMD and ABM (see Proposition 7.3 and Theorem 7.4 ).

The third goal of this paper is to show in $\S 9$ a Lipschitz continuity of the BMD complex Poisson kernel $\Psi(z, \zeta)$ under the perturbation of the standard slit domains, which combined with the continuity theorems obtained in $\S 8$ will enable us to derive the continuity of the right-hand side of (1.4) in $t$ and consequently the desired differentiability of $g_{t}(z)$ in $t$. The BMD complex Poisson kernel can be obtained from the classical Green function through operations of taking normal derivatives at $\partial \mathbb{H}$, taking periods around the slits and taking line integrals of normal derivatives along smooth curves. We shall thus utilize two perturbation formulae of Green's function whose proof will be given in Appendix 3 by following the interior variation method in Garabedian [10] of constructing a special parametrix of an elliptic partial differential operator for a transformed Green function and solving an associated Fredholm type integral equation. One of these formulae was considered in 2 in relation to slit motions, but we shall make them considerably more detailed and precise to be usable for the present purpose.

\section{BROWNIAN MOTION WITH DARNING}

A subset $A$ of $\mathbb{R}^{2}$ is said to be a continuum if $A$ is a connected closed set containing at least two points. Suppose $A$ is a continuum. Then, due to a Lebesgue's theorem, $A$ is non-polar and each point $z \in A$ is a regular point of $A$ with respect to the two-dimensional Brownian motion $Z=\left(Z_{t}, \mathbb{P}_{x}\right)$ (cf. [17]). In particular, for any $\alpha \geq 0$ and any bounded continuous function $f$ on $A$, the function

$$
w(z):=\mathbb{E}_{z}\left[e^{-\alpha \sigma_{A}} f\left(Z_{\sigma_{A}}\right)\right], \quad z \in \mathbb{R}^{2},
$$


is a bounded continuous function on $\mathbb{R}^{2}$ with (cf. [17, Proposition 2.3.5])

$$
\lim _{z^{\prime} \rightarrow z, z^{\prime} \in \mathbb{R}^{2} \backslash A} w\left(z^{\prime}\right)=f(z) \text { for } z \in \partial\left(\mathbb{R}^{2} \backslash A\right), \quad w(z)=f(z) \text { for } z \in A .
$$

Here $\sigma_{A}$ denotes the first hitting time of $A$ by the process $Z$; that is, $\sigma_{A}:=$ $\inf \left\{t>0: Z_{t} \in A\right\}$. We will use similar notation for other processes, such as $Z^{*}$, and its meaning should be clear from the context.

Consider a domain $E \subset \mathbb{R}^{2}$. We assume $E$ is $\mathbb{R}^{2}$ or otherwise $\mathbb{R}^{2} \backslash E$ consists of continuum components. Let

$$
D=E \backslash K, \quad K=\bigcup_{i \in \Lambda} A_{i}
$$

where $\left\{A_{i}, i \in \Lambda\right\}$ is a finite or a countably infinite family of mutually disjoint compact continuum contained in $E$ that are locally finite on $\mathbb{R}^{2}$.

Let

$$
D^{*}=D \cup K^{*}, \quad K^{*}=\left\{a_{i}^{*}, i \in \Lambda\right\},
$$

and define a neighborhood $U_{i}^{*}$ of each point $a_{i}^{*}$ in $D^{*}$ by $\left\{a_{i}^{*}\right\} \cup\left(U_{i} \backslash A_{i}\right)$ for some neighborhood $U_{i}$ of $A_{i}$ in $E$. That is, $D^{*}$ is a topological space obtained from $E$ by regarding each continuum $A_{i}$ as one point $a_{i}^{*}$. Denote by $m$ the Lebesgue measure in $D$ that is extended to $D^{*}$ by setting $m\left(K^{*}\right)=0$.

Definition 2.1. Brownian motion with darning (abbreviated as BMD) $Z^{*}=$ $\left(Z_{t},{ }^{*}, \zeta^{*}, \mathbb{P}_{z}^{*}\right)$ is an $m$-symmetric diffusion on $D^{*}$ such that

(i) its subprocess killed upon leaving $D$ has the same law as Brownian motion in $D$;

(ii) it admits no killings on $K^{*}$.

Recall that a Markov process $Z^{*}$ is said to be $m$-symmetric if the transition semigroup of $Z^{*}$ is self-adjoint in $L^{2}\left(D^{*} ; m\right)$ and that $Z^{*}$ admits no killings on $K^{*}$ means $\mathbb{P}_{x}\left(Z_{\zeta^{*}-}^{*}=a^{*}, \zeta^{*}<\infty\right)=0$ for $m$-a.e. $x \in D^{*}$. Here $\zeta^{*}$ denotes the lifetime of $Z^{*}$. In Definition 2.1, assuming (i), then it is easy to see that condition (ii) is equivalent to

$$
\mathbb{P}_{x}\left(Z_{\zeta^{*}-}^{*}=a^{*}, \zeta^{*}<\infty\right)=0 \quad \text { for every } x \in D .
$$

Observe that it follows from the $m$-symmetry of $Z^{*}$ and the fact that $m\left(K^{*}\right)=0$ that BMD $Z^{*}$ spends zero Lebesgue amount of time (i.e. zero sojourn time) at $K^{*}$. We point out that $D$ can be disconnected as $E \backslash A_{i}$ can be disconnected.

As a consequence of a more general result [5, Theorems 7.7.3 and 7.7.4], BMD on $D^{*}$ exists and is unique in law. (In fact, BMD can be defined in any dimension as in [4,5].) For the reader's convenience, following [4], we present below a direct proof of this fact through two theorems.

Remark 2.2. The BMD coincides with the excursion reflected Brownian motion (ERBM) described by Drenning [7] when $K$ consists of just one single continuum $A$ so that $\mathbb{C} \backslash A$ is connected. In the case that $E=\mathbb{C}$ and $A=\mathbb{D}$, the unit disk centered at the origin, a Feller transition semigroup $\left\{T_{t}, t \geq 0\right\}$ of the ERBM on $(\mathbb{C} \backslash \mathbb{D}) \cup\left\{a^{*}\right\}$ whose associated Hunt process extends the absorbing Brownian motion on $\mathbb{C} \backslash \mathbb{D}$ was explicitly given in [7, Proposition 3.1]. It is easy to see that $T_{t}$ is $m$-symmetric. Therefore the ERBM must be equal to the BMD due to the stated uniqueness of the BMD. Based on Lawler's description of ERBM in the case 
of $E=\mathbb{C}$ and $A=\mathbb{D}$ using excursion laws, identification between BMD and ERBM is also done in [5, Remark 7.6.4]. The ERBM for a general open set $E$ and a continuum $A \subset E$ so that $\mathbb{C} \backslash A$ is connected is defined in [7] by a conformal map from $\mathbb{C} \backslash \mathbb{D}$ with a due time change and a killing upon leaving $E$. Hence it can also be identified with the BMD due to the conformal invariance of BMD formulated in [5. $\S 7.8(1)]$. However, even in this one single continuum case, BMD is more general as, for BMD, $\mathbb{C} \backslash A$ is allowed to have disconnected components, for example, when $A$ is the unit circle in $\mathbb{C}$.

Consider next the case that $\Lambda=\{1, \cdots, N\}$ for $N \geq 2$ and each $\mathbb{C} \backslash A_{i}$ is connected. Then, by the locality (4, Theorem 1.3.1]) and conformal invariance (5.) Theorem 7.8.1]) of BMD, our BMD $Z^{*}$ on $D^{*}$ has the properties described in [7. §3.4] and enjoys all the properties prescribed in [7, Definition 3.1] for ERBM. So $Z^{*}$ may be identified with the ERBM if some uniqueness statement is available concerning [7, Definition 3.1].

Let $Z=\left(Z_{t}, \mathbb{P}_{z}\right)$ (resp. $Z^{0}=\left(Z_{t}^{0}, \mathbb{P}_{z}^{0}\right)$ ) be an absorbing Brownian motion (abbreviated as ABM) on $E$ (resp. on $D)$. The Dirichlet form for $Z$ on $L^{2}(E ; d x)$ (resp. for $Z^{0}$ on $\left.L^{2}(D ; d x)\right)$ is $\left(\mathbf{D}, W_{0}^{1,2}(E)\right)$ (resp. $\left.\left(\mathbf{D}, W_{0}^{1,2}(D)\right)\right)$. Here for an open set $E, W_{0}^{1,2}(E)$ is the $\mathbf{D}_{1}^{1 / 2}$-completion of the space $C_{c}^{\infty}(E)$ of smooth functions with compact support in $E$, where $\mathbf{D}$ denotes the Dirichlet integral on $E$ and $\mathbf{D}_{1}(u, u):=\mathbf{D}(u, u)+\int_{E} u(x)^{2} d x$.

Define for $i \in \Lambda$ and $z \in E$,

$$
u^{(i)}(z)=\mathbb{E}_{z}\left[e^{-\sigma_{K}} ; Z_{\sigma_{K}} \in A_{i}\right], \quad \varphi^{(i)}(z)=\mathbb{P}_{z}\left(Z_{\sigma_{K}} \in A_{i}\right) .
$$

We call the system $\left\{\varphi^{(i)} ; i \in \Lambda\right\}$ the harmonic basis for the domain $D$. In complex analysis, $\varphi^{(i)}$ is called the harmonic measure of $A_{i}$. By (2.1), both $u^{(i)}$ and $\varphi^{(i)}$ are continuous functions on $\bar{E}$ that take constant value 1 on $A_{i}$ and vanish on $\partial E$ and on $A_{j}$ for $j \neq i$.

Now we define

$$
\mathcal{F}^{*}=\mathbf{D}_{1} \text {-closure of linear span of } C_{c}^{\infty}(D) \text { and }\left\{\left.u^{(j)}\right|_{D} ; i \in \Lambda\right\},
$$

and for $u, v \in \mathcal{F}^{*}$,

$$
\mathcal{E}^{*}(u, v)=\frac{1}{2} \int_{D} \nabla u(x) \cdot \nabla v(x) d x
$$

Observe that

$$
\mathcal{F}^{*}=\left\{\left.u\right|_{D}: u \in W_{0}^{1,2}(E), u \text { is constant D-q.e. on each } A_{j}\right\} .
$$

It is easy to check that $\left(\mathcal{E}^{*}, \mathcal{F}^{*}\right)$ is a Dirichlet form on $L^{2}(D ; d x)=L^{2}\left(D^{*} ; m\right)$.

Theorem 2.3. The quadratic form $\left(\mathcal{E}^{*}, \mathcal{F}^{*}\right)$ defined by (2.5)-(2.6) is a regular Dirichlet form on $L^{2}\left(D^{*} ; m\right)$. It is strongly local and each $a_{j}^{*}$ has positive capacity. Consequently, there is an $m$-symmetric diffusion $Z^{*}$ on $D^{*}$ that starts from every point in $D^{*}$ and admits no killings on $D^{*}$. The diffusion $Z^{*}$ is BMD on $D^{*}$ and every $a_{j}^{*}$ is regular for itself (that is, $\mathbb{P}_{a^{*}}\left(\sigma_{\left\{a^{*}\right\}}=0\right)=1$ ).

Proof. Let $\mathcal{C}$ be the linear span of $C_{c}^{\infty}(D)$ and $\left\{u^{(j)} ; j \in \Lambda\right\}$. By defining $u\left(a_{j}^{*}\right)$ to be the value of $u$ on $A_{j}$, we can view $\mathcal{C}$ as a subspace of $C\left(D^{*}\right) \cap \mathcal{F}^{*}$. Since $\mathcal{C}$ is an algebra that separates points in $D^{*}$, by the Weierstrass theorem, $\mathcal{C}$ is uniformly dense in the space $C_{\infty}\left(D^{*}\right)$ of continuous functions in $D^{*}$ vanishing at $\partial D^{*}$. Clearly 
$\mathcal{C}$ is $\mathcal{E}_{1}^{*}$-dense in $\mathcal{F}^{*}$. Hence $\left(\mathcal{E}^{*}, \mathcal{F}^{*}\right)$ is a regular Dirichlet form on $L^{2}\left(D^{*} ; m\right)$. It is strongly local and its part Dirichlet form on $D$ is $\left(\mathbf{D}, W_{0}^{1,2}(D)\right)$. So there is an $m$-symmetric diffusion $Z^{*}$ on $D^{*}$ associated with $\left(\mathcal{E}^{*}, \mathcal{F}^{*}\right)$, whose part process in $D$ is the killed Brownian motion in $D$. The diffusion $Z^{*}$ is a BMD on $D^{*}$. Since Brownian motion $X^{E}$ in $E$ starting from $x \in D$ visits each $A_{j}$ with positive probability, $Z^{*}$ starting from $x \in D$ visits each $a_{j}^{*}$ with positive probability. This implies that each $a_{j}^{*}$ has positive capacity. Consequently, $Z^{*}$ can be refined to start from every point in $D^{*}$. That each $a_{j}^{*}$ is regular for itself follows from the general fact that for any nearly Borel measurable set $A, A \backslash A^{r}$ is semipolar and hence $m$-polar.

Theorem 2.4. BMD on $D^{*}$ is unique in law.

Proof. It suffices to show that if $Z^{*}$ is a BMD on $D^{*}$, its associated quasi-regular Dirichlet form $(\mathcal{E}, \mathcal{F})$ on $L^{2}\left(D^{*} ; m\right)$ has to be $\left(\mathcal{E}^{*}, \mathcal{F}^{*}\right)$. First note that according to the definition of BMD, each $a_{j}^{*}$ is non-polar for $Z^{*}$, and that the part Dirichlet form $\left(\mathcal{E}, \mathcal{F}_{D}\right)$ of $(\mathcal{E}, \mathcal{F})$ in $D$ is $\left(\mathbf{D}, W_{0}^{1,2}(D)\right.$ ) (see [5, Theorem 3.3.8]). By the $\mathcal{E}_{1}$-orthogonal projection (see [5, Theorem 3.2.2]), for every $u \in \mathcal{F}, \mathbf{H}_{K^{*}}^{1} u(x):=$ $\mathbb{E}_{x}\left[e^{-\sigma^{*}} u\left(Z_{\sigma^{*}}^{*}\right)\right] \in \mathcal{F}$ and $u-\mathbf{H}_{K^{*}}^{1} u \in W_{0}^{1,2}(D)$. Here $K^{*}:=\left\{a_{i}^{*} ; i \in \Lambda\right\}$ and $\sigma^{*}:=\inf \left\{t>0: Z_{t}^{*} \in K^{*}\right\}$. Now

$$
\mathbf{H}_{K^{*}}^{1} u(x)=\sum_{j \in \Lambda} u\left(a_{j}^{*}\right) \mathbb{E}_{x}\left[e^{-\sigma^{*}} ; Z_{\sigma^{*}}^{*}=a_{j}^{*}\right] \quad \text { for } x \in D .
$$

By the continuity of $Z^{*}$, the definition of $a_{j}^{*}$ and the fact that $X^{*, D}$ has the same distribution as the subprocess of $X^{E}$ killed upon leaving $D$, we see that

$$
\mathbb{E}_{x}\left[e^{-\sigma^{*}} ; Z_{\sigma^{*}}^{*}=a_{j}^{*}\right]=\mathbb{E}_{x}\left[e^{-\sigma_{E}} ; X_{\sigma_{K}}^{E} \in A_{j}\right]=u^{(j)}(x) \text { for } x \in D .
$$

It then follows that $\mathbf{H}_{K^{*}}^{1} u=\sum_{j \in \Lambda} u\left(a_{j}^{*}\right) u^{(j)}(x)$. As each $a_{j}^{*}$ is non-polar, for every finite subset $\Lambda_{1}$ of $\Lambda$ having $N$ elements,

$$
\left\{\left(u\left(a_{j}^{*}\right), j \in \Lambda_{1}\right) ; u \in \mathcal{F}\right\}=\mathbb{R}^{N},
$$

and so $\mathcal{F}=\mathcal{F}^{*}$. Note that $(\mathcal{E}, \mathcal{F})$ is strongly local, so for every bounded $u \in \mathcal{F}=$ $\mathcal{F}^{*}$,

$$
\mathcal{E}(u, u)=\frac{1}{2} \mu_{\langle u\rangle}^{c}\left(D^{*}\right)=\frac{1}{2} \mu_{\langle u\rangle}^{c}(D)+\sum_{j \in \Lambda} \mu_{\langle u\rangle}^{c}\left(a_{j}^{*}\right)=\frac{1}{2} \mu_{\langle u\rangle}^{c}(D),
$$

where in the last inequality we used the fact that the energy measure $\mu_{\langle u\rangle}^{c}$ of $u$ does not charge on level sets of $u$ (cf. [5, Theorem 4.3.8]). As $\left(\mathcal{E}, \mathcal{F}_{D}\right)=\left(\mathbf{D}, W_{0}^{1,2}(D)\right)$, we have by the strong local property of $\mu_{\langle u\rangle}^{c}$ (see [5, Proposition 4.3.1]) that $\mu_{\langle u\rangle}^{c}(d x)=|\nabla u(x)|^{2} d x$ on $D$. Consequently $\mathcal{E}(u, u)=\frac{1}{2} \int_{D}|\nabla u(x)|^{2} d x$ for every bounded and hence for any $u \in \mathcal{F}$. This completes the proof for $(\mathcal{E}, \mathcal{F})=$ $\left(\mathcal{E}^{*}, \mathcal{F}^{*}\right)$.

It is easy to see from the definition of BMD and the conformal invariance of Brownian motion that BMD is invariant under the conformal map up to a time change. See [5, Theorem 7.8.1]. 


\section{ZERO PERIOD PROPERTY OF BMD-HARMONIC FUNCTIONS}

From now on, we assume that the index set $\Lambda$ is a finite set $\{1, \ldots, N\}$, and so $K=\bigcup_{i=1}^{N} A_{i}$.

3.1. BMD harmonicity and its locality. Denote by $Z=\left(Z_{t}, \mathbb{P}_{z}\right), Z^{0}=\left(Z_{t}^{0}, \mathbb{P}_{z}^{0}\right)$ the $\mathrm{ABM}$ on $E$ and $D=E \backslash K$, respectively, and by $Z^{*}=\left(Z_{s}, \mathbb{P}_{z}^{*}\right)$ the BMD on the set

$$
D^{*}=D \cup K^{*}, \quad K^{*}=\left\{a_{1}^{*}, \cdots, a_{N}^{*}\right\},
$$

obtained from $Z$ by regarding each hole $A_{i}$ as one point $a_{i}^{*}$.

A function $u$ defined on a connected open subset $O$ of $D^{*}$ is said to be $Z^{*}$ harmonic or BMD-harmonic on $O$ (with respect to $Z^{*}$ ) if $u$ is continuous on $O$ and, for any relatively compact open set $O_{1}$ with $\bar{O}_{1} \subset O$,

$$
\mathbb{E}_{z}^{*}\left[\left|u\left(Z_{\tau_{O_{1}}}^{*}\right)\right|\right]<\infty \quad \text { and } \quad \mathbb{E}_{z}^{*}\left[u\left(Z_{\tau_{O_{1}}}^{*}\right)\right]=u(z) \quad \text { for every } z \in O_{1} .
$$

Here $\tau_{O_{1}}=\inf \left\{t \geq 0: Z_{t}^{*} \notin O_{1}\right\}$ is the first exit time from $O_{1}$ by $Z^{*}$. The restriction to $O \cap D$ of any $Z^{*}$-harmonic function on $O$ is harmonic there in the classical sense (with respect to Brownian motion) and takes constant boundary value $u\left(a_{i}^{*}\right)$ at $\partial A_{i}$ whenever $a_{i}^{*} \in O$.

Theorem 3.1. Suppose that $D_{1}$ and $D_{2}$ are two connected subsets of $D^{*}$ and that $D_{1} \cap D_{2} \neq \emptyset$. If $u$ is $Z^{*}$-harmonic in $D_{i}$ for $i=1,2$, then $u$ is $Z^{*}$-harmonic in $D_{1} \cup D_{2}$.

Proof. Let $O$ be a relatively compact open subset of $D_{1} \cup D_{2}$. Let $\left\{U_{k}^{(i)} ; k \geq 1\right\}$ be an increasing sequence of relatively compact open subsets whose union is $D_{i}$ and $\partial U_{k}^{(i)}$ is a smooth subset in $D$ for $i=1,2$. Since $\left\{U_{k}^{(1)} \cup U_{k}^{(2)} ; k \geq 1\right\}$ forms an open cover for $\bar{O}$, there is some $k_{0} \geq 1$ so that $\bar{O} \subset U_{k_{0}}^{(1)} \cup U_{k_{0}}^{(2)}$. For notational simplicity, denote $U_{k_{0}}^{(i)}$ by $U_{i}$ for $i=1,2$. Note that $O_{i}:=O \cap U_{i}$ is a relatively compact open subset of $D_{i}, i=1,2$. We claim that for every $x \in O, u(x)=\mathbb{E}_{x}\left[u\left(Z_{\tau_{O}}^{*}\right)\right]$. In the following we show that the above holds for every $x \in O_{1}$. The case for $x \in O_{2}$ is analogous.

Let $\left\{\theta_{t} ; t \geq 0\right\}$ be the shift operator for BMD $Z^{*}$ on $D^{*}$. We use $\left\{\mathcal{F}_{t} ; t \geq 0\right\}$ to denote the minimal augmented natural filtration generated by $Z^{*}$. Define a sequence of stopping times as follows: $T_{1}:=\tau_{O_{1}}, T_{2}:=\tau_{O_{2}}$, and for $k \geq 1$,

$$
T_{2 k+1}:=T_{2 k}+\tau_{O_{1}} \circ \theta_{T_{2 k}} \text { and } T_{2 k+2}:=T_{2 k+1}+\tau_{O_{2}} \circ \theta_{T_{2 k+1}} .
$$

Note that $\tau_{O}<\infty$ and $T_{k} \leq \tau_{O}$ for every $k \geq 1$. Since $u$ is $Z^{*}$-harmonic in both $D_{1}$ and $D_{2}$, we have for $x \in O_{1}, \mathbb{P}_{x}$-a.s.,

$$
u\left(X_{T_{k}}\right)=\mathbb{E}_{Z_{T_{k+1}^{*}}^{*}}\left[u\left(Z_{T_{k+1}}^{*}\right) \mid \mathcal{F}_{T_{k}}\right] \quad \text { for every } k \geq 1 .
$$

In other words, $\left\{u\left(Z_{T_{k}}^{*}\right) ; k \geq 1\right\}$ is an $\left\{\mathcal{F}_{T_{k}}\right\}_{k \geq 1}$-filtration under $\mathbb{P}_{x}$ for every $x \in O_{1}$. Let $T:=\lim _{k \rightarrow \infty} T_{k}$. Since $u$ is bounded and continuous on $\bar{O}$, we have

$$
u(x)=\lim _{k \rightarrow \infty} \mathbb{E}_{x}\left[u\left(Z_{T_{k}}^{*}\right)\right]=\mathbb{E}_{x}\left[u\left(Z_{T}^{*}\right)\right] .
$$

We next show that $T=\tau_{O}$. Clearly $T \leq \tau_{O} \mathbb{P}_{x}$-a.s. On $\left\{T<\tau_{O}\right\}, Z_{T}^{*}(\omega) \in O=$ $O_{1} \cup O_{2}$, say $Z_{T}^{*}(\omega) \in O_{2}$. There is some large $k_{0}=k_{0}(\omega)$ so that $Z_{T_{k}}^{*}(\omega) \in O_{2}$ for all $k \geq k_{0}$. This is impossible, as for even $k \geq k_{0}, Z_{T_{k}}^{*} \notin O_{2}$. So we must have 
$T=\tau_{O} \mathbb{P}_{x}$-a.s. and, consequently, $u(x)=\mathbb{E}_{x}\left[u\left(Z_{\tau_{O}}^{*}\right)\right]$ for every $x \in O_{1}$. This shows that $u$ is $Z^{*}$-harmonic in $O$ for every relatively compact subdomain $O$ of $D_{1} \cup D_{2}$, and so $u$ is $Z^{*}$-harmonic in $D_{1} \cup D_{2}$.

Consider an open set $\widetilde{E}$ such that $\widetilde{E} \subset E, \mathbb{C} \backslash \widetilde{E}$ is a continuum and each compact continuum $A_{i}$ is either contained in $\widetilde{E}$ or disjoint from $\widetilde{E}$. Let $\widetilde{K}=\widetilde{E} \cap K=\bigcup_{j=1}^{\ell} A_{i_{j}}$ for $1 \leq i_{1}<\cdots<i_{\ell}, \ell \leq N$, and $\widetilde{D}=\widetilde{E} \backslash \widetilde{K}$. Denote by $\widetilde{Z}^{*}$ the BMD on the set

$$
\widetilde{D}^{*}=\widetilde{D} \cup \widetilde{K}^{*}, \quad \widetilde{K}^{*}=\left\{a_{i_{1}}^{*}, \cdots, a_{i_{\ell}}^{*}\right\},
$$

obtained from the ABM on $\widetilde{E}$ by rendering each hole $A_{i_{j}}$ as a one point $a_{i_{j}}^{*} 1 \leq j \leq \ell$. Then $\widetilde{Z}^{*}$ is identical in law with the part process $Z_{\widetilde{D}^{*}}^{*}$ of $Z^{*}$ on $\widetilde{D}^{*}$, namely, the subprocess of $Z^{*}$ obtained by killing upon its exit time from $\widetilde{D}^{*}$. This is because both $\widetilde{Z}^{*}$ and $Z_{\widetilde{D}^{*}}^{*}$ are symmetric diffusion extensions of $\mathrm{ABM}$ on $\widetilde{D}$ to $\widetilde{D}^{*}$ admitting no killing on $\widetilde{K}^{*}$, and the uniqueness result stated in $\S 2$ applies. Let $O$ be an open connected subset of $\widetilde{D}^{*}\left(\subset D^{*}\right)$. A function $u$ on $O$ is $Z^{*}$-harmonic on $O$ if and only if $u$ is harmonic with respect to the part process $Z_{\widetilde{D}^{*}}^{*}$ of $Z^{*}$ on $\widetilde{D}^{*}$. Therefore we have the following equivalence:

$$
u \text { is } Z^{*} \text {-harmonic on } O \Longleftrightarrow u \text { is } \widetilde{Z}^{*} \text {-harmonic on } O \text {. }
$$

3.2. Zero flux condition for generator of BMD. Let $\left(\mathcal{A}^{*}, \mathcal{D}\left(\mathcal{A}^{*}\right)\right)$ denote the $L^{2}$-infinitesimal generator of $\mathrm{BMD} Z^{*}$ or, equivalently, of the Dirichlet form $\left(\mathcal{E}^{*}, \mathcal{F}^{*}\right)$. That is, $u \in \mathcal{D}\left(\mathcal{A}^{*}\right)$ if and only if $u \in \mathcal{F}^{*}$ and there is some $f \in$ $L^{2}(D ; d x)=L^{2}\left(D^{*} ; m\right)$ so that

$$
\mathcal{E}^{*}(u, v)=-\int_{D} f(x) v(x) d x \quad \text { for every } v \in \mathcal{F}^{*} .
$$

We denote the above $f$ as $\mathcal{A}^{*} u$. In view of (2.5), condition (3.4) is equivalent to

$$
\frac{1}{2} \int_{D} \nabla u(x) \cdot \nabla v(x) d x=-\int_{D} f(x) v(x) d x \quad \text { for every } v \in C_{c}^{\infty}(D)
$$

and

$$
\frac{1}{2} \int_{D} \nabla u(x) \cdot \nabla u_{j}(x) d x=-\int_{D} f(x) u_{j}(x) d x \quad \text { for every } j=1, \ldots, N .
$$

(3.5) says that $\Delta u$ exists on $D$ in the distributional sense and $f=\frac{1}{2} \Delta u \in L^{2}(D ; d x)$. Let us define the flux $\mathcal{N}(u)\left(a_{j}^{*}\right)$ of $u$ at $a_{j}^{*}$ by

$$
\mathcal{N}(u)\left(a_{j}^{*}\right)=\int_{D} \nabla u(x) \cdot \nabla u_{j}(x) d x+\int_{D} \Delta u(x) u_{j}(x) d x .
$$

Then (3.6) is equivalent to

$$
\mathcal{N}(u)\left(a_{j}^{*}\right)=0 \quad \text { for every } j=1, \ldots, N .
$$

Hence we have established the following.

Theorem 3.2. A function $u \in \mathcal{F}^{*}$ is in $\mathcal{D}\left(\mathcal{A}^{*}\right)$ if and only if the distributional Laplacian $\Delta u$ of $u$ exists as an $L^{2}$-integrable function on $D$ and $u$ has zero flux at every $a_{j}^{*}$. Moreover, for $u \in \mathcal{D}\left(\mathcal{A}^{*}\right), \mathcal{A}^{*} u=\frac{1}{2} \Delta u$ on $D$. 
Note that when $\partial K_{j}$ is smooth for $j=1, \ldots, N$, then by the Green-Gauss formula we have

$$
\mathcal{N}(u)\left(a_{j}^{*}\right)=\int_{\partial K} \frac{\partial u(x)}{\partial \mathbf{n}} u^{(j)}(x) \sigma(d x),
$$

where $\mathbf{n}$ is the unit outward normal vector field of $D$ on $\partial D$ and $\sigma$ is the surface measure on $\partial D$. Since $u^{(j)}(x)=1$ on $K_{j}$ and $u^{(j)}(x)=0$ on $K_{i}$ with $i \neq j$,

$$
\mathcal{N}(u)\left(a_{j}^{*}\right)=\int_{\partial K_{j}} \frac{\partial u(x)}{\partial \mathbf{n}} \sigma(d x)
$$

For $\alpha \geq 0$, we use $G_{\alpha}^{*}$ to denote the $\alpha$-order resolvent of $Z^{*}$; that is, $G_{\alpha}^{*} f(x)=$ $\mathbb{E}_{x}\left[\int_{0}^{\infty} e^{-\alpha t} f\left(Z_{t}^{*}\right) d t\right]$ for $f \geq 0$ on $D^{*}$. Throughout this paper, we use the convention that $\partial$ is a cemetery point added to $D^{*}, Z_{t}^{*}=\partial$ for $t \geq \zeta^{*}$ and that every function $f$ defined on $D^{*}$ is extended to $\partial$ by setting $f(\partial)=0$. When $\alpha=0$, we will simply denote $G_{0}^{*}$ by $G^{*}$.

Lemma 3.3. If $E$ is bounded, then for every $f \in L^{\infty}(D)\left(=L^{\infty}(D ; m)\right), G^{*} f \in$ $\mathcal{D}\left(\mathcal{A}^{*}\right)$ with $\mathcal{A}^{*} G^{*} f=-f$.

Proof. Since $D=E \backslash K$ is bounded, $G^{0}\left(L^{\infty}(D)\right) \subset L^{\infty}(D) \subset L^{2}(D)$ for the 0-order resolvent $G^{0}$ of $Z^{0}$ in view of (4.6) below with $w_{D}=0$. $G^{*}$ has the same property because for $f \in L^{\infty}(D), G^{*} f$ is a linear combination of $G^{0} f$ and $\varphi^{(i)}$ for $1 \leq i \leq N$. Hence the resolvent equation $G^{*} f=G_{1}^{*} f+G_{1}^{*}\left(G^{*} f\right)$ yields that $G^{*} f \in \mathcal{D}\left(\mathcal{A}^{*}\right)$ with $\mathcal{A}^{*} G^{*} f=-f$.

3.3. Zero period property of BMD-harmonic functions. In this subsection, we assume that $E$ is a planar domain whose complement is a continuum and $A_{1}, \cdots A_{N}$ are disjoint compact continua contained in $E$ so that each $E \backslash A_{j}$ is connected. As before, $K=\bigcup_{j=1}^{N} A_{j}$. The domain $D:=E \backslash K$ is called $(N+1)$ connected. In what follows, we mostly consider an $(N+1)$-connected domain with $E$ being the upper half-plane $\mathbb{H}$. An $(N+1)$-connected domain $D=E \backslash K$ is called a standard slit domain if $E=\mathbb{H}$ and $A_{i} \subset \mathbb{H}$ is a line segment parallel to the $x$-axis for each $1 \leq i \leq N$.

Let $\gamma$ be a $C^{1}$-smooth simple curve surrounding $A_{j}$, namely, $\gamma \subset D$, ins $\gamma \supset A_{j}$, $\overline{\operatorname{ins} \gamma} \cap A_{k}=\emptyset, k \neq j$. Here ins $\gamma$ denotes the bounded component of $\mathbb{C} \backslash \gamma$ and is called the interior of $\gamma$. For a harmonic function $u$ defined in a neighborhood of $A_{j}$, the value

$$
\int_{\gamma} \frac{\partial u(\zeta)}{\partial \mathbf{n}_{\zeta}} d s(\zeta)
$$

is independent of the choice of such curve $\gamma$ with $\mathbf{n}$ denoting the normal vector pointing toward $A_{j}$ and $s$ the arc length of $\gamma$. This value is called the period of $u$ around $A_{j}$.

Theorem 3.4. Let $O$ be a connected open subset of $D^{*}$. A $Z^{*}$-harmonic function in $O$ has zero period around $A_{i}$ for every $i$ with $a_{i}^{*} \in O$.

Proof. The assertion trivially holds if $O$ does not contain any $a_{i}^{*}$. In view of Theorem 3.1 and the equivalence (3.3), without loss of generality, we may and do assume that $E$ is bounded with smooth boundary $\partial E, D^{*}=O$ and that $D^{*}$ contains exactly one $a_{1}^{*}$ (that is, $K$ consists of exactly one compact continuum $A_{1}$ ).

Suppose that $v$ is $Z^{*}$-harmonic in $D^{*}$. Clearly $v$ is harmonic in $D$. Let $U_{1}$ and $U_{2}$ be relatively compact open subsets of $E$ so that $A_{1} \subset U_{1} \subset \bar{U}_{1} \subset U_{2} \subset \bar{U}_{2} \subset E$. 
Let $\psi \in C_{c}^{\infty}\left(\mathbb{R}^{2}\right)$ so that $\psi=1$ on $U_{1}$ and $\psi=0$ on $U_{2}^{c}$. Define $f(x)=-\frac{1}{2} \Delta(\psi v)(x)$ for $x \in D$. Note that $f \in L^{\infty}(D ; d x)$ and $f=0$ on $D \backslash\left(U_{2} \backslash U_{1}\right)$. Hence $G^{*} f$ is $Z^{*}$ harmonic in $\left(U_{1} \cap D\right) \cup\left\{a_{1}^{*}\right\}$ and so is $w:=\psi v-G^{*} f$. On the other hand, Lemma 3.3 implies that $w$ is harmonic and hence $Z^{*}$-harmonic in $D$. Thus by Theorem 3.1, $w$ is $Z^{*}$-harmonic in $D^{*}$. Since both $\psi v$ and $G^{*} f$ vanish on $\partial E=\partial D^{*}, w=0$ on $\partial D^{*}$. Thus by the maximum principle for the bounded $Z^{*}$-harmonic function $w$ on $D^{*}$ (note that $a_{1}^{*}$ is an interior point of $D^{*}$ ), we have $w=0$ on $D^{*}$, that is, $\psi v=G^{*} f$ on $D^{*}$.

Let $u_{1}(x):=\mathbb{E}_{x}\left[e^{-\sigma_{A_{1}}}\right]$, which is smooth, strictly smaller than 1 on $D$, and continuous on $E$ with value 1 on $A_{1}$. For $\varepsilon \in(0,1)$, let $\eta_{\varepsilon}$ be the boundary of the connected component of $\left\{x \in E: u_{1}(x)>1-\varepsilon\right\}$ that contains $A_{1}$. By Sard's theorem (see, e.g., [15]), there is a set $\mathcal{N}_{0}$ having zero Lebesgue measure so that for every $\varepsilon \in(0,1) \backslash \mathcal{N}_{0}, \eta_{\varepsilon}$ is $C^{\infty}$-smooth. Take a decreasing sequence $\left\{\varepsilon_{n}, n \geq 1\right\} \in(0,1) \backslash \mathcal{N}_{0}$ with $\lim _{n \rightarrow \infty} \varepsilon_{n}=0$. Since $\left\{x \in E: u_{1}(x)>1-\varepsilon_{n}\right\}$ decreases to $A_{1}$, we may assume that each $\eta_{\varepsilon_{n}}$ is contained inside $U_{1}$. As $\psi=1$ on $U_{1}$, we can see by the Green-Gauss formula, Theorem 3.2 and Lemma 3.3 that the period of $v$ at $a_{1}^{*}$ equals

$$
\begin{aligned}
& \lim _{n \rightarrow \infty} \int_{\eta_{\varepsilon_{n}}} \frac{\partial(\psi v)(\xi)}{\partial \mathbf{n}_{\xi}} \sigma(d \xi)=\lim _{n \rightarrow \infty} \frac{1}{1-\varepsilon_{n}} \int_{\eta_{\varepsilon_{n}}} \frac{\partial G^{*} f(\xi)}{\partial \mathbf{n}_{\xi}} u_{1}(\xi) \sigma(d \xi) \\
= & \lim _{n \rightarrow \infty} \frac{1}{1-\varepsilon_{n}} \int_{D \backslash \operatorname{ins}\left(\eta_{n}\right)}\left(\nabla u_{1} \cdot \nabla G^{*} f+u_{1} \Delta G^{*} f\right) d x \\
= & \int_{D} \nabla u_{1}(x) \cdot \nabla G^{*} f(x) d x+\int_{D} u_{1}(x) \Delta G^{*} f(x) d x=2 \mathcal{N}\left(G^{*} f\right)\left(a_{1}^{*}\right)=0 .
\end{aligned}
$$

Here $\mathbf{n}$ denotes the unit inward normal vector field on $\eta_{\varepsilon_{n}}$ for the interior of $\eta_{\varepsilon_{n}}$.

Remark 3.5. Theorem 3.2, Lemma 3.3 and Theorem 3.4 in fact hold in any dimension. Moreover, the statement in Theorem 3.4 is in fact a characterization for $v$ to be $Z^{*}$-harmonic in $O$; see [4] for details.

By the zero period property of $v$, the value of

$$
u(z)=-\int_{\gamma} \frac{\partial v(\xi)}{\partial \mathbf{n}_{\xi}} \sigma(d \xi)
$$

is independent of the choice of the smooth $C^{2}$ simple curve $\gamma$ that joins a fixed $z_{0}$ to $z$, and $f(z):=u(z)+i v(z)$ is an analytic function in $D$. Hence we obtain

Corollary 3.6. If $v$ is $Z^{*}$-harmonic on $D^{*}$, then $-\left.v\right|_{D}$ admits a harmonic conjugate $u$ on $D$ uniquely up to an additive real constant so that $f(z)=u(z)+i v(z), z \in$ $D$, is an analytic function on $D$.

\section{Green function, Harmonic FunCtions And their PERIOdS}

We first consider a planar domain $D$ whose complement is non-empty and consists of continuum components. We recall the relationship between the 0-order resolvent density of the ABM $Z^{0}$ on $D$ and the classical Green function. Denote by $p_{t}^{0}(z, \zeta), z, \zeta \in D$, the transition density of the $\mathrm{ABM} Z^{0}$ on $D$ and $\tau_{D}:=\inf \left\{t>0: Z_{t} \notin D\right\}$ the first exit time of the Brownian motion $Z=\left(Z_{t}, \mathbb{P}_{z}\right)$ from $D$. Since $Z$ has transition density function $n(t, z-\zeta)$ with respect to the 
Lebesgue measure on $\mathbb{R}^{2}$, where $n(t, z)=(2 \pi t)^{-1} \exp \left(-\frac{|z|^{2}}{2 t}\right)$, it follows from the strong Markov property of $Z$ that

$$
p_{t}^{0}(z, \zeta)=n(t, z-\zeta)-\mathbb{E}_{z}\left[n\left(t-\tau_{D}, Z_{\tau_{D}}-\zeta\right) ; \tau_{D}<t\right] .
$$

The resolvent density $G_{\alpha}^{0}(z, \zeta)$ and the 0 -order resolvent density $G^{0}(z, \zeta)$ of $Z^{0}$ are defined respectively by

$$
G_{\alpha}^{0}(z, \zeta)=\int_{0}^{\infty} e^{-\alpha t} p_{t}^{0}(z, \zeta) d t \quad \text { and } \quad G^{0}(z, \zeta)=\int_{0}^{\infty} p_{t}^{0}(z, \zeta) d t, \quad z, \zeta \in D .
$$

The integral $\int_{0}^{\infty} n(t, z) d t$ is infinite, but the re-centered integral

$$
\begin{aligned}
\int_{0}^{\infty}\left(n(t, z)-n\left(t, \mathbf{e}_{1}\right)\right) d t & =\int_{0}^{\infty} \frac{1}{2 \pi t}\left(e^{-|z|^{2} / 2 t}-e^{-1 / 2 t}\right) d t \\
& =-\frac{1}{\pi} \log |z| \quad \text { for } z \in \mathbb{C}
\end{aligned}
$$

is finite where $\mathbf{e}_{1}=(1,0) \in \mathbb{C}$. Since $n(t, z) \geq n\left(t, \mathbf{e}_{1}\right)$ when $|z| \leq 1$ and $n(t, z)<$ $n\left(t, \mathbf{e}_{1}\right)$ when $|z|>1$, in fact we have from (4.2) that

$$
\int_{0}^{\infty}\left|n(t, z)-n\left(t, \mathbf{e}_{1}\right)\right| d t=\frac{1}{\pi}|\log | z|| \quad \text { for } z \in \mathbb{C} .
$$

Moreover, we know from [17, Theorem 3.4.2] and Harnack's inequality that

$$
\mathbb{E}_{z}\left[|\log | Z_{\tau_{D}}-\zeta||\right]<\infty \quad \text { for every } z, \zeta \in D .
$$

From (4.1), one has

$p_{t}^{0}(z, \zeta)=n(t, z-\zeta)-n\left(t, \mathbf{e}_{1}\right)-\mathbb{E}_{z}\left[n\left(t-\tau_{D}, Z_{\tau_{D}}-\zeta\right)-n\left(t-\tau_{D}, \mathbf{e}_{1}\right) ; \tau_{D}<t\right]$

$$
+n\left(t, \mathbf{e}_{1}\right)-\mathbb{E}_{z}\left[n\left(t-\tau_{D}, \mathbf{e}_{1}\right) ; \tau_{D}<t\right] .
$$

Hence it follows from (4.3)-(4.4) that for distinct $z, \zeta \in D$,

$$
\begin{aligned}
& \int_{0}^{\infty}\left|n\left(t, \mathbf{e}_{1}\right)-\mathbb{E}_{z}\left[n\left(t-\tau_{D}, \mathbf{e}_{1}\right) ; \tau_{D}<t\right]\right| d t \\
\leq & G^{0}(z, \zeta)+\frac{1}{\pi}|\log | z-\zeta||+\frac{1}{\pi} \mathbb{E}_{z}\left[|\log | Z_{\tau_{D}}-\zeta||\right]<\infty .
\end{aligned}
$$

Let $W_{D}(z)=\int_{0}^{\infty} \mathbb{E}_{z}\left[n\left(t, \mathbf{e}_{1}\right)-n\left(t-\tau_{D}, \mathbf{e}_{1}\right) \mathbf{1}_{\left\{t>\tau_{D}\right\}}\right] d t$. Then it follows from (4.5) that

$$
G^{0}(z, \zeta)=-\frac{1}{\pi} \log |z-\zeta|+\frac{1}{\pi} \mathbb{E}_{z} \log \left|Z_{\tau_{D}}-\zeta\right|+W_{D}(z), \quad z, \zeta \in D .
$$

By [17, Proposition 4.5.4], for each $\zeta \in D$, as a function of $z, G^{0}(z, \zeta)+\frac{1}{\pi} \log |z-\zeta|$ is harmonic in $D$ and thus so is $W_{D}(z)$. Note that

$$
\begin{aligned}
W_{D}(z) & =\lim _{N \rightarrow \infty} \int_{0}^{N} \mathbb{E}_{z}\left[n\left(t, \mathbf{e}_{1}\right)-n\left(t-\tau_{D}, \mathbf{e}_{1}\right) \mathbf{1}_{\left\{t>\tau_{D}\right\}}\right] d t \\
& =\lim _{N \rightarrow \infty} \mathbb{E}_{z}\left[\int_{0}^{N} n\left(t, \mathbf{e}_{1}\right) d t-\int_{0}^{N \vee \tau_{D}-\tau_{D}} n\left(s, \mathbf{e}_{1}\right) d s\right] \\
& =\lim _{N \rightarrow \infty} \mathbb{E}_{z}\left[\int_{N \vee \tau_{D}-\tau_{D}}^{N} n\left(t, \mathbf{e}_{1}\right) d t\right] \geq 0 .
\end{aligned}
$$

From the last display, we get the following monotonicity property on $W$ : if $D_{1} \supset D_{2}$, then $W_{D_{1}}(z) \geq W_{D_{2}}(z)$ for every $z \in D_{2}$. 
Denote by $\mathbb{H}$ the upper half-plane $\{z=x+i y \in \mathbb{C}: y>0\}$ and by $G^{\mathbb{H}, 0}(z, \zeta)$ the 0 -order resolvent density of the $\mathrm{ABM} Z^{\mathbb{H}, 0}$ in $\mathbb{H}$. One deduces easily from (4.2) and the identity $p_{t}^{\mathbb{H}, 0}(z, \zeta)=n(t, z-\zeta)-n(t, z-\bar{\zeta})$ that

$$
G^{\mathbb{H}, 0}(z, \zeta)=\frac{1}{\pi}(\log |z-\bar{\zeta}|-\log |z-\zeta|) .
$$

By (4.6), $W_{\mathbb{H}}(z)=\frac{1}{\pi}\left(\log |z-\bar{\zeta}|-\mathbb{E}_{z} \log \left|Z_{\tau_{\mathbb{H}}}-\zeta\right|\right)$. Taking $\zeta=i y$ and letting $y \rightarrow+\infty$, one concludes from the above display that $W_{\mathbb{H}} \equiv 0$. Consequently, for any $D \subset \mathbb{H}$, we have by the monotonicity that $W_{D}=0$ on $D$ and

$$
G^{0}(z, \zeta)=-\frac{1}{\pi} \log |z-\zeta|+\frac{1}{\pi} \mathbb{E}_{z} \log \left|Z_{\tau_{D}}-\zeta\right|, \quad z, \zeta \in D .
$$

In other words, the 0-order resolvent density of $Z^{0}$ coincides with the classical Green function multiplied by the constant $\frac{1}{\pi}$ whenever the domain $D$ is contained in a half space. For this reason, we call the 0-order resolvent density of the ABM $Z^{0}$ on $D$ also its Green function. We point out that $W_{D}$ can be non-trivial for some unbounded $D$. For example, when $D=\{z \in \mathbb{C}:|z|>r\}$ for $r>0$, $W_{D}(z)=\frac{1}{\pi} \log ^{+}(|z| / r)$ (cf. [17, Proposition 4.9]). We take this opportunity to point out that some condition is needed for Proposition 2.36 of [13] to hold for a regular domain $D$ in $\mathbb{R}^{2}$.

The bounded harmonic function in $\mathbb{H} \backslash \mathbb{D}$ with boundary values 1 on $\partial \mathbb{D}$ and 0 on $\mathbb{R}$ tends to zero at infinity. By the maximum principle, we easily obtain

Lemma 4.1. Let $D=\mathbb{H} \backslash K$ be an $(N+1)$-connected domain.

(i) The function $\varphi^{(j)}(z), z \in D$, defined by (2.4) with $E=\mathbb{H}$ is harmonic in $D$ and satisfies

$$
\lim _{|z| \rightarrow \infty, z \in D} \varphi^{(j)}(z)=0, \quad j \geq 1
$$

(ii) For any continuous function $g$ on $\partial \mathbb{H}$ with compact support, the function

$$
\psi_{g}(z):=\mathbb{E}_{z}^{0}\left[g\left(Z_{\zeta^{0}-}^{0}\right) ; Z_{\zeta^{0}-}^{0} \in \partial \mathbb{H}\right], \quad z \in D,
$$

is a harmonic function in $D$ and satisfies

$$
\lim _{|z| \rightarrow \infty, z \in D} \psi_{g}(z)=0 .
$$

Here $\zeta^{0}$ denotes the lifetime of the $A B M Z^{0}$ on $D$.

Lemma 4.2. Denote the positive $x$-axis and the positive $y$-axis by $\Gamma_{0}$ and $\Lambda_{0}$, respectively. Let $Z=\left(Z_{t}, \mathbb{P}_{z}\right)$ be the Brownian motion on $\mathbb{C}$. Then

$$
\mathbb{P}_{z}\left(\sigma_{\Lambda_{0}}<\sigma_{\Gamma_{0}}\right)=\frac{2}{\pi} \tan ^{-1}(y / x) \quad \text { for } z=x+i y \text { with } x>0, y>0 .
$$

Proof. The conformal map $\phi(z)=z^{2}$ of the first quadrant onto the upper halfplane maps $\Lambda_{0}$ onto $(-\infty, 0)$ and $\Gamma_{0}$ onto $(0, \infty)$. Hence the lemma follows from the conformal invariance of the absorbing Brownian motion and the formula

$$
\omega(z, \mathbb{H},[a, b])=\frac{1}{\pi} \arg \frac{z-b}{z-a}
$$

for the harmonic measure of an interval $(a, b)$ in $\mathbb{H}$; see [11, I (1.1)]. 
Lemma 4.3. For $a>0$, consider the rectangle $R_{a}:=\{z=x+i y:-a<x<$ $a, 0<y<a\}$ and put $\Sigma_{a}=\partial R_{a} \backslash \partial \mathbb{H}$. Let $h$ be a harmonic function on $\mathbb{H} \backslash R_{\ell_{0}}$ for some $\ell_{0}>0$ such that

$$
\sup _{\zeta \in \mathbb{H} \backslash R_{\ell_{0}}}|h(\zeta)|:=C<\infty, \quad h(x+i 0+)=0 \quad \text { for any } x \text { with }|x|>\ell_{0} .
$$

Then

$$
\int_{\Sigma_{\ell}}\left|\frac{\partial h(\zeta)}{\partial \mathbf{n}_{\zeta}}\right| d s(\zeta) \leq 8 C \quad \text { for any } \ell>2 \ell_{0} .
$$

The estimate (4.14) follows readily from the Poisson formulae for the harmonic function $h$ on the half-planes $\left\{z \in \mathbb{C}: y>\ell_{1}\right\}$ and $\left\{z \in \mathbb{C}: x>\ell_{1}\right\}$. The latter reads, for $x>\ell_{1}, y>0$,

$$
h(x+i y)=\frac{1}{\pi} \int_{-\infty}^{\infty} \frac{x-\ell_{1}}{\left(y-y^{\prime}\right)^{2}+\left(x-\ell_{1}\right)^{2}} h\left(\ell_{1}+i y^{\prime}\right) d y^{\prime},
$$

where $h$ is extended from $\left\{z \in \mathbb{C}: x>\ell_{0}, y>0\right\}$ to $\left\{z \in \mathbb{C}: x>\ell_{0}\right\}$ by the Schwarz reflection.

We will also need the following; see for instance [11, Theorem II.2.3] for the standard proof of (i). The second part easily follows by approximation of $\mathbb{H}$ with bounded domains, using Lemma 4.3

Lemma 4.4. Let $D=\mathbb{H} \backslash \bigcup_{i=1}^{N} A_{i}$ be an $(N+1)$-connected domain and $f$ be a harmonic function on $D$.

(i) Take mutually disjoint $C^{1}$-smooth simple curves $\gamma_{i}$ surrounding $A_{i}, 1 \leq$ $i \leq N$, and an analytic Jordan curve $\Gamma \subset \mathbb{H}$ containing $\bigcup_{i=1}^{N}$ ins $\gamma_{i}$. Then, for any $z \in$ ins $\Gamma \backslash \bigcup_{i=1}^{N}$ ins $\gamma_{i}$,

$$
\begin{aligned}
f(z)= & -\frac{1}{2} \sum_{k=1}^{N} \int_{\gamma_{k}} \frac{\partial G^{0}(z, \zeta)}{\partial \mathbf{n}_{\zeta}} f(\zeta) d s(\zeta)+\frac{1}{2} \sum_{k=1}^{N} \int_{\gamma_{k}} G^{0}(z, \zeta) \frac{\partial f(\zeta)}{\partial \mathbf{n}_{\zeta}} d s(\zeta) \\
& -\frac{1}{2} \int_{\Gamma} \frac{\partial G^{0}(z, \zeta)}{\partial \mathbf{n}_{\zeta}} f(\zeta) d s(\zeta)+\frac{1}{2} \int_{\Gamma} G^{0}(z, \zeta) \frac{\partial f(\zeta)}{\partial \mathbf{n}_{\zeta}} d s(\zeta) .
\end{aligned}
$$

(ii) Suppose further that a harmonic function $f$ on $D$ takes a smooth boundary function with compact support on $\partial \mathbb{H}$ and satisfies

$$
\lim _{|\zeta| \rightarrow \infty, \zeta \in D} f(\zeta)=0 .
$$

Then, for analytic smooth simple curves $\gamma_{i}, 1 \leq i \leq N$, as in (i) and for any $z \in \mathbb{H} \backslash \bigcup_{i=1}^{N} \gamma_{i}$,

$$
\begin{aligned}
f(z)= & -\frac{1}{2} \sum_{k=1}^{N} \int_{\gamma_{k}} \frac{\partial G^{0}(z, \zeta)}{\partial \mathbf{n}_{\zeta}} f(\zeta) d s(\zeta)+\frac{1}{2} \sum_{k=1}^{N} \int_{\gamma_{k}} G^{0}(z, \zeta) \frac{\partial f(\zeta)}{\partial \mathbf{n}_{\zeta}} d s(\zeta) \\
& -\frac{1}{2} \int_{\partial \mathbb{H}} \frac{\partial G^{0}(z, \zeta)}{\partial \mathbf{n}_{\zeta}} f(\zeta) d s(\zeta) .
\end{aligned}
$$

Theorem 4.5. Let $D=\mathbb{H} \backslash \bigcup_{i=1}^{N} A_{i}$ be an $(N+1)$-connected domain.

(i) For each $z \in D, 1 \leq i \leq N,-2 \varphi^{(i)}(z)$ equals the period of the harmonic function $G^{0}(z, \cdot)$ around $A_{i}$. 
(ii) For any bounded continuous function $g$ on $\partial \mathbb{H}$,

$$
\mathbb{E}_{z}^{0}\left[g\left(Z_{\zeta^{0}-}^{0}\right) ; Z_{\zeta^{0}-}^{0} \in \partial \mathbb{H}\right]=-\frac{1}{2} \int_{\partial \mathbb{H}} \frac{\partial G^{0}(z, \zeta)}{\partial \mathbf{n}_{\zeta}} g(\zeta) d s(\zeta), z \in D .
$$

(iii) Let $f$ be a harmonic function on $D$ taking a constant value $f_{i}$ on each $\partial A_{i}$, taking a continuous boundary function with compact support on $\partial \mathbb{H}$ and satisfying

$$
\lim _{|z| \rightarrow \infty, z \in D} f(z)=0 .
$$

It then holds for every $z \in D$ that

$$
f(z)=\sum_{k=1}^{N} f_{k} \varphi^{(k)}(z)-\frac{1}{2} \int_{\partial \mathbb{H}} \frac{\partial G^{0}(z, \zeta)}{\partial \mathbf{n}_{\zeta}} f(\zeta) d s(\zeta) .
$$

It also holds that for each $1 \leq i \leq N$,

$$
\text { the period of } f \text { around } A_{i}=\sum_{k=1}^{N} f_{k} a_{k i}+\int_{\partial \mathbb{H}} \frac{\partial \varphi^{(i)}(\zeta)}{\partial \mathbf{n}_{\zeta}} f(\zeta) d s(\zeta),
$$

where $a_{k i}$ denotes the period of $\varphi^{(k)}$ around $A_{i}$.

Proof. (i) If the $A_{i}$ are closed analytic curves, the claim follows from Lemma 4.4 with $f=\varphi^{(i)}$. In general, approximate $A_{i}$ by the level sets $\left\{G^{0}(z, \zeta)=a\right\}$ which are closed analytic curves for almost all values of $a>0$. Since the Green function $G_{a}^{0}$ of the domain $D_{a}=\left\{z: G^{0}(z, \zeta)>a\right\}$ satisfies $G_{a}^{0}=G^{0}-a$, the claim follows from the weak convergence of harmonic measure of $D_{a}$ to the harmonic measure of $D$; see [11, II Exercise 4].

(ii) can be proved similarly by applying Lemma 4.4 to the function $f$ which coincides with $g$ on $\partial \mathbb{H}$ and which is zero on $\partial D_{a} \backslash \partial \mathbb{H}$. Since Lemma 4.4 assumes smoothness of the boundary function, it should be applied to a mollification $g_{\epsilon}$ of $g$, rather than $g$ itself. Passing to the limit $\epsilon \rightarrow 0$ poses no difficulties.

(iii) Let $h$ be the difference of the functions on both sides of (4.21). Then $h$ is a harmonic function on $D$ taking value 0 on $\bigcup_{i=1}^{N} A_{i}$ and on $\partial \mathbb{H}$ as well. By condition (4.20) and Lemma 4.1] $\lim _{|z| \rightarrow \infty, z \in D} h(z)=0$. Hence the maximum principle applies to $h$ as in [16, p. 9], and we can conclude that $h=0$.

Finally (4.22) follows from (4.21) by taking the periods around $A_{i}$ on both sides of (4.21) and by noting (i) and the symmetry of $G^{0}$.

\section{Green function and Poisson kernel of BMD}

Since

$$
\mathbb{P}_{z}^{*}\left(\zeta^{*}<\infty, Z_{\zeta^{*}-}^{*} \in \partial \mathbb{H}\right) \geq \mathbb{P}_{z}^{0}\left(\zeta^{0}<\infty, Z_{\zeta^{0}-}^{0} \in \partial \mathbb{H}\right)>0, z \in D,
$$

$Z^{*}$ is transient. Denote by $G^{*}$ the 0 -order resolvent of $Z^{*}$ :

$$
G^{*} f(z)=\mathbb{E}_{z}^{*}\left[\int_{0}^{\infty} f\left(Z_{s}\right) d t\right], \quad z \in D^{*}, f \in \mathcal{B}_{+}\left(D^{*}\right) .
$$

Lemma 5.1. For any Borel measurable function $f \geq 0$ on $D^{*}$,

$$
G^{*} f(z)=\int_{D} G^{*}(z, \zeta) f(\zeta) m(d \zeta)
$$


where

$$
G^{*}(z, \zeta)=G^{0}(z, \zeta)+2 \Phi(z) \mathcal{A}^{-1} \Phi(\zeta)^{t r} \quad z \in D^{*}, \zeta \in D
$$

Here $\Phi(z)=\left(\varphi^{(1)}(z), \cdots, \varphi^{(N)}(z)\right), \mathcal{A}$ is an $N \times N$-matrix whose $(i, j)$-component $a_{i j}$ is the period of $\varphi^{(j)}$ around $A_{i}, 1 \leq i, j \leq N$, and the superscript "tr" denotes a vector transpose.

Proof. Let $f \in C_{c}(D)$. Then $G^{*} f \in \mathcal{F}^{*}$ is $Z^{*}$-harmonic in $D^{*} \backslash \operatorname{supp}[f]$ and

$$
G^{*} f(z)=G^{0} f(z)+\sum_{i=1}^{N} \varphi^{(i)}(z) \lambda_{i}, \quad \text { where } \lambda_{i}=G^{*} f\left(a_{i}^{*}\right) .
$$

By Theorem 3.4, we have for a smooth simple curve $\gamma_{j}$ surrounding $A_{j}$,

$$
\frac{1}{2} \sum_{i=1}^{N} a_{i j} \lambda_{i}=-\frac{1}{2} \int_{\gamma_{j}} \frac{\partial G^{0} f(\zeta)}{\partial \mathbf{n}_{\zeta}} d s(\zeta), \quad 1 \leq i, j \leq N .
$$

Since the right-hand side in the above equals $\left(\varphi^{(j)}, f\right)$ in view of the symmetry of $G^{0}$, and Theorem 4.5(i), it suffices to solve the above linear equation in $\lambda_{i}, 1 \leq i \leq$ $N$.

We call $G^{*}(z, \zeta), z \in D^{*}, \zeta \in D$, of Lemma 5.1 the Green function of $Z^{*}$. One can deduce the symmetry of the matrix $\breve{\mathcal{A}}$ from the symmetry of the Green functions of $Z^{0}$ and $Z^{*}$. We now define the Poisson kernel of $Z^{*}$ by

$$
K^{*}(z, \zeta)=-\frac{1}{2} \frac{\partial}{\partial \mathbf{n}_{\zeta}} G^{*}(z, \zeta), z \in D^{*}, \zeta \in \partial \mathbb{H}
$$

Note that

$$
K^{*}(z, \zeta)=-\frac{1}{2} \frac{\partial}{\partial \mathbf{n}_{\zeta}} G^{0}(z, \zeta)-\Phi(z) \mathcal{A}^{-1} \frac{\partial}{\partial \mathbf{n}_{\zeta}} \Phi(\zeta)^{t r}, z \in D^{*}, \zeta \in \partial \mathbb{H} .
$$

Thus for each $\zeta \in \partial \mathbb{H}, z \mapsto K^{*}(z, \zeta)$ is a $Z^{*}$-harmonic function of $z$ on $D^{*}$.

Lemma 5.2. $\quad$ (i) For any closed interval $J \subset \partial \mathbb{H}, K^{*}(z, \zeta)$ can be uniquely extended to a jointly continuous function on $(\overline{\mathbb{H}} \backslash J) \times J$.

(ii) For any $g \in b C(\partial \mathbb{H})$, the integral

$$
H^{*} g(z)=\int_{\partial \mathbb{H}} K^{*}(z, \zeta) g(\zeta) d s(\zeta), \quad z \in D^{*}
$$

gives a well-defined bounded $Z^{*}$-harmonic function on $D^{*}$ and

$$
\lim _{z \rightarrow \zeta, z \in D} H^{*} g(z)=g(\zeta), \quad \zeta \in \partial \mathbb{H} .
$$

(iii) It holds that

$$
\lim _{z \rightarrow \infty} K^{*}(z, \zeta)=0 \quad \text { uniformly in } \zeta \text { on any compact interval of } \partial \mathbb{H} \text {. }
$$

(iv) It holds for any $g \in b C(\partial \mathbb{H})$ that

$$
\mathbb{E}_{z}^{*}\left[g\left(Z_{\zeta^{*}-}^{*}\right)\right]=H^{*} g(z), \quad x \in D^{*},
$$

where $\zeta^{*}$ denotes the lifetime of $Z^{*}$. 
Proof. (i) Note that every point in $C_{j}$ (resp. $\partial \mathbb{H}$ ) is a regular point for $C_{j}$ (resp. $\partial \mathbb{H})$. Thus $G^{0}(\cdot, \zeta)$ extends to be a continuous function on $\overline{\mathbb{H}} \backslash\{\zeta\}$ by taking values 0 on $\partial \mathbb{H} \cup K$, and $\varphi^{(k)}$ extends to be a continuous function on $\overline{\mathbb{H}}$ by taking values $\delta_{k j}$ on $A_{j}$ and 0 on $\partial \mathbb{H}$. Since $G^{0}(z, \zeta)$ and $\varphi^{(k)}(\zeta), 1 \leq k \leq N$, are, as functions of $\zeta$, harmonic in $D$ and vanish on $\partial \mathbb{H}$, they admit harmonic extensions across $\partial \mathbb{H}$ by the Schwarz reflection. Now by the integral representation of harmonic functions in balls in terms of Poisson kernels, the right-hand side of (5.2) is jointly continuous on the desired set.

(ii) Since $\varphi^{(k)}$ vanishes on $\partial \mathbb{H},\left.\frac{\partial \varphi^{(j)}(x+i y)}{\partial y}\right|_{y=0} \geq 0$ for every $x \in \mathbb{R}$. For every non-negative function $g$ on $\partial \mathbb{H}$, we have by (4.19) and (5.2)

$$
H^{*} g(z)=\mathbb{E}_{z}^{0}\left[g\left(Z_{\zeta^{0}-}^{0}\right)\right]+\left.\sum_{i, j=1}^{N} \varphi^{(i)}(z)\left(\mathcal{A}^{-1}\right)_{i j} \int_{-\infty}^{\infty} \frac{\partial \varphi^{(j)}(x+i y)}{\partial y}\right|_{y=0} g(x) d x .
$$

So it suffices to show that

$$
\left.\int_{-\infty}^{\infty} \frac{\partial \varphi^{(k)}(x+i y)}{\partial y}\right|_{y=0} d x \leq 2 \nu^{(k)}\left(A_{k}\right)
$$

To verify (5.6), consider the ABM $Z^{\mathbb{H}, 0}=\left(Z_{t}^{\mathbb{H}, 0}, \mathbb{P}_{z}^{\mathbb{H}, 0}\right)$ on the upper half-plane $\mathbb{H}$ and let $\psi^{(k)}(z)=\mathbb{P}_{z}^{\mathbb{H}, 0}\left(\sigma_{A_{k}}<\infty\right), z \in \mathbb{H}, 1 \leq k \leq N$. The function $\psi^{(k)}$ is a 0-order equilibrium potential of the compact set $A_{k}$. So by Corollary 3.4.3 and the 0 -order version of Lemma 2.3.10 of [5], there is a finite positive measure $\nu^{(k)}$ concentrated on $A_{k}$ so that

$$
\psi^{(k)}(z)=\int_{A_{k}} G^{\mathbb{H}, 0}\left(z, z^{\prime}\right) d \nu^{(k)}\left(z^{\prime}\right), \quad z \in \mathbb{H} .
$$

Here $G^{\mathbb{H}, 0}\left(z, z^{\prime}\right)$ is the Green function of $Z^{\mathbb{H}, 0}$ given by (4.7).

The measure $\nu^{(k)}$ is called the 0 -order equilibrium measure of $A_{k}$ relative to $Z^{\mathbb{H}, 0}$, and $\nu^{(k)}\left(A_{k}\right)$ is the (0-order) capacity of $A_{k}$ in $\mathbb{H}$ (cf. [5]). Since $\varphi^{(k)} \leq \psi^{(k)}$, we have

$$
\left.\frac{\partial \varphi^{(k)}(x+i y)}{\partial y}\right|_{y=0} \leq \frac{2}{\pi} \int_{A_{k}} \frac{y^{\prime}}{\left(x-x^{\prime}\right)^{2}+\left(y^{\prime}\right)^{2}} \nu^{(k)}\left(d z^{\prime}\right),
$$

which yields 5.6 .

(iii) In view of the domination $G^{0}(z, \zeta) \leq G^{\mathbb{H}, 0}(z, \zeta)$ and (4.7), we have for $z=x+i y \in D, \zeta=\xi+i 0 \in \partial \mathbb{H}$,

$$
-\frac{1}{2} \frac{\partial}{\partial \mathbf{n}_{\zeta}} G^{0}(z, \zeta) \leq \frac{1}{\pi} \frac{y}{(x-\xi)^{2}+y^{2}} .
$$

Hence the first term of the expression (5.2) of $K^{*}(z, \zeta)$ converges to 0 as $z \rightarrow \infty$ uniformly in $\zeta$ on any compact interval of $\partial \mathbb{H}$, and so does its second term due to (4.9) and the continuity of $\frac{\partial \varphi^{(j)}(\zeta)}{\partial \mathbf{n}_{\zeta}}$ in $\zeta \in \partial \mathbb{H}$ for every $1 \leq j \leq N$. Thus we get (5.4).

(iv) It is enough to prove (5.5) for a continuous function $g$ on $\partial \mathbb{H}$ with compact support. Both sides of (5.5) are $Z^{*}$-harmonic functions with the same boundary value $g(\zeta), \zeta \in \partial \mathbb{H}$. As $z \rightarrow \infty$, the right-hand side vanishes by (5.4), and so does the left-hand side because it is a linear combination of $\psi_{g}(z)$ of (4.10) and $\varphi^{(i)}(z), 1 \leq i \leq N$, and (4.9) and (4.11) apply. Hence (5.5) holds by the maximum principle. 


\section{Complex Poisson kernel of BMD AND THE CHORDAl Komatu-LOEWNER EQUATION}

For $z \in \mathbb{C}$ and $r>0$, we use either $B_{r}(z)$ or $B(z, r)$ to denote the open ball with radius $r$ centered at $z$.

6.1. Complex Poisson kernel of BMD on a slit domain. Let $D=\mathbb{H} \backslash$ $\left\{C_{1}, \cdots, C_{N}\right\}$ be a standard slit domain and $K^{*}(z, \zeta), z \in D^{*}, \zeta \in \partial \mathbb{H}$, be the Poisson kernel (5.2) of the BMD on $D^{*}$. As $K^{*}(z, \zeta)$ is BMD-harmonic in $z \in D$ for each fixed $\zeta \in \partial \mathbb{H}$, by Corollary 3.6 there is an analytic function $\Psi(z, \zeta)$, unique up to an additive real constant, having $K^{*}(z, \zeta)$ as its imaginary part.

Lemma 6.1. (i) The limit $\lim _{z \rightarrow \infty} \Psi(z, \zeta)$ exists and is real-valued. Furthermore $\lim \sup _{z \rightarrow \infty} \sup _{\zeta \in J}|\Psi(z, \zeta)|<\infty$ for any compact interval $J \subset \partial \mathbb{H}$.

(ii) $\Psi(z, \zeta)$ is determined uniquely by the normalization condition

$$
\lim _{z \rightarrow \infty} \Psi(z, \zeta)=0 \text {. }
$$

$\Psi(z, \zeta)$ is then jointly continuous in $(z, \zeta)$ on $\left(D \cup\left(\cup_{i=1}^{N}\left(C_{i}^{+} \cup C_{i}^{-}\right)\right) \cup(\partial \mathbb{H} \backslash J)\right)$ $\times J$. Here $J$ is any compact subinterval of $\partial \mathbb{H}$ and $C_{i}^{+}$(resp. $C_{i}^{-}$) denotes the upper (resp. lower) side of the (closed) slit $C_{i}$ equipped with the topology induced from the path distance in $D$.

Proof. (i) By (5.7), we have

$$
-\left.\frac{1}{2} \frac{\partial}{\partial y} \frac{\partial}{\partial \mathbf{n}_{\zeta}} G^{0}(z, \zeta)\right|_{y=0} \leq \frac{1}{\pi} \frac{1}{(x-\xi)^{2}},
$$

which combined with the expression (5.2) and (5.6) implies that for any interval $I \subset \partial \mathbb{H}$ containing $\zeta$,

$$
\int_{\partial \mathbb{H} \backslash I}\left|\frac{\partial K^{*}(x+i y, \zeta)}{\partial y}\right|_{y=0} d x \text { is finite and continuous in } \zeta \in I .
$$

Since $z \mapsto \Psi(z, \zeta)$ is an analytic function on $D$ whose imaginary part vanishes on $\partial \mathbb{H} \backslash \zeta$ by (5.2) and (5.7), it can be extended to an analytic function on $E=$ $\mathbb{C} \backslash\left(\bigcup_{k=1}^{N} C_{k}\right) \cup\left(\bigcup_{k=1}^{N} \pi\left(C_{k}\right)\right) \backslash\{\zeta\}$ by the reflection principle, where $\pi$ denotes the reflection with respect to $\partial \mathbb{H}$. Denote the extended analytic function $z \mapsto \Psi(z, \zeta)$ as $u(z)+i v(z)$ with $v(z)=K^{*}(z, \zeta)$. The real part $u$ can then be evaluated by

$$
u(z)-u\left(z_{0}\right)=\int_{C} v_{y} d x-v_{x} d y\left(=-\int_{C} \frac{\partial v\left(z^{\prime}\right)}{\partial \mathbf{n}_{z^{\prime}}} d s\left(z^{\prime}\right)\right),
$$

where $C$ is any smooth simple curve connecting $z_{0}$ with $z$ in $D$. We fix an arbitrary compact interval $J \subset \partial \mathbb{H}$ and take $\zeta \in J, z_{0} \in \partial \mathbb{H} \backslash J$. Choose $\ell_{0}>0$ with $J \subset\left(-\ell_{0}, \ell_{0}\right), \bigcup_{i=1}^{N} C_{i} \subset R_{\ell_{0}}$.

By (5.4), we see that $v(z)=K^{*}(z, \zeta)$ is bounded on $\mathbb{H} \backslash R_{\ell_{0}}$ uniformly in $\zeta \in J$. Therefore by Lemma 4.3 .

$$
\sup _{\zeta \in J, \ell>2 \ell_{0}} \int_{\Sigma_{\ell}}\left|\frac{\partial K^{*}(z, \zeta)}{\partial \mathbf{n}_{z}}\right| d s(z)<\infty .
$$

For $z \in \mathbb{H} \backslash R_{2 \ell_{0}}$, let $\ell \geq 2 \ell_{0}$ be such that $z \in \Sigma_{l}$ and $C$ be a smooth simple curve connecting $z_{0}$ to $z$ in $D$. By the zero period property of $v$, the integral of $\frac{\partial v\left(z^{\prime}\right)}{\partial \mathbf{n}_{z^{\prime}}} d s\left(z^{\prime}\right)$ along a closed Jordan curve consisting of $C$, a part of $\Sigma_{\ell}$ and a part 
of the $x$-axis vanishes. Hence we can deduce from Lemma 4.3 and (6.3) that $u(z)$ is bounded near $\infty$ uniformly in $\zeta \in J$. This combined with (5.4) yields the second assertion of (i).

In particular, the analytic function $\Psi\left(\frac{1}{z}\right)$ is uniformly bounded near the origin $\mathbf{0}$, which is therefore a removable singularity of this analytic function near $\mathbf{0}$, yielding the first assertion of (i).

(ii) Write $\Psi(z, \zeta)=u(z, \zeta)+i K^{*}(z, \zeta)$. By Lemma 5.2(i), $K^{*}(z, \zeta)$ is continuous in $(z, \zeta)$ on $\left[\mathbb{C} \backslash \bigcup_{k=1}^{N}\left(C_{k} \cup \pi C_{k}\right) \backslash J\right] \times J$, and so are $\frac{\partial}{\partial x} K^{*}(z, \zeta)$ and $\frac{\partial}{\partial y} K(z, \zeta)$ because $K^{*}(z, \zeta)$ is harmonic in $z$. Fix some $z_{0}=x_{0}+i 0 \in \partial \mathbb{H} \backslash J$ located to the right of $J . A s \lim _{x \rightarrow \infty} u(x+i 0, \zeta)=0$ by (6.1), we see from (6.4) that $u(z, \zeta)$, $z \in D \cup(\partial \mathbb{H} \backslash J), \zeta \in J$, is determined by

$u(z, \zeta)=\int_{C}-\frac{\partial}{\partial y} K^{*}(x+i y, \zeta) d x+\frac{\partial}{\partial x} K^{*}(x+i y, \zeta) d y+\int_{x_{0}}^{\infty} \frac{\partial}{\partial y} K^{*}(x+i 0, \zeta) d x$,

for any smooth simple curve $C$ connecting $z_{0}$ to $z$ in $D \cup(\partial \mathbb{H} \backslash J)$. Consequently, for any $z_{1}, z_{2} \in D \cup(\partial \mathbb{H} \backslash J)$,

$$
u\left(z_{2}, \zeta\right)-u\left(z_{1}, \zeta\right)=\int_{C} \frac{\partial}{\partial y} K^{*}(x+i y, \zeta) d x-\frac{\partial}{\partial x} K^{*}(x+i y, \zeta) d y
$$

for a smooth simple curve $C$ joining $z_{1}$ to $z_{2}$ through $D \cup(\partial \mathbb{H} \backslash J)$. The joint continuity of $u(x, \zeta)$ on $(D \cup \partial \mathbb{H} \backslash J) \times J$ follows from these two formulae and (6.3).

Since, as a function of $z, K^{*}(z, \zeta)$ takes a constant boundary value on each slit $C_{i}$, it can be extended to be a harmonic function and hence a smooth function across $C_{j}^{+}$ and $C_{j}^{-}$except at the endpoints for every $j=1, \ldots, N$. It follows from the above that $u(z, \zeta)$ can be extended continuously to $C_{j}^{+}$and $C_{j}^{-}$except at the endpoints for every $j=1, \ldots, N$. We next show that $u(z, \zeta)$ can also be extended to the endpoints of $C_{j}$. Let $z_{1}$ be the left endpoint of $C_{j}$. Take $\varepsilon>0$ small so that it is less than one half of the length of $C_{j}$ and that $B\left(z_{1}, \varepsilon\right) \backslash C_{j} \subset D$. Then $\psi(z)=\left(z-z_{1}\right)^{1 / 2}$ maps $B\left(z_{1}, \varepsilon\right) \backslash C_{j}$ conformally onto $B(0, \sqrt{\varepsilon}) \cap \mathbb{H}$. Clearly $f(z, \zeta):=K^{*}\left(z^{2}+z_{1}, \zeta\right)$ is a harmonic function in $z \in B(0, \sqrt{\varepsilon}) \cap \mathbb{H}$ that is continuous up to $B(0, \sqrt{\varepsilon}) \cap$ $\partial \mathbb{H}$ and takes a constant value there. Hence by the Schwarz reflection principle, $f(z, \zeta)$ can be extended to a harmonic function in $z \in B(0, \sqrt{\varepsilon})$ and so is smooth there. Moreover, by the integral representation of harmonic functions in disks using Poisson kernels, $\nabla_{z} f(z, \zeta)$ is in fact jointly continuous in $(z, \zeta) \in B(0, \sqrt{\varepsilon}) \times \partial \mathbb{H}$. This combined with (6.5) shows that $z \mapsto u(z, \zeta)$ can be extended continuously to $z_{1}$ and the resulting function is jointly continuous in $(z, \zeta)$. The case for the right endpoint of $C_{j}$ can be dealt with analogously. This completes the proof of the lemma.

We call $\Psi(z, \zeta), z \in D, \zeta \in \partial \mathbb{H}$, subjected to the normalization condition (6.1) the complex Poisson kernel of BMD for the standard slit domain $D$.

6.2. The map $g_{t, s}$ and an expression of $a_{t}-a_{s}$. We are in a position to consider a fixed standard slit domain $D=\mathbb{H} \backslash\left\{C_{1}, \cdots, C_{N}\right\}$ together with a Jordan arc

$$
\gamma:\left[0, t_{\gamma}\right] \rightarrow \bar{D}, \quad \gamma(0) \in \partial \mathbb{H}, \quad \gamma\left(0, t_{\gamma}\right] \subset D
$$


For each $t \in\left[0, t_{\gamma}\right]$, let $g_{t}$ be a conformal map from $D \backslash \gamma(0, t]$ onto a standard slit domain $D_{t}$ satisfying the condition

$$
g_{t}(z)=z+\frac{a_{t}}{z}+o\left(\frac{1}{|z|}\right), \quad z \rightarrow \infty .
$$

$a_{t}$ is then a real-valued function of $t$.

The unique existence of such a map $g_{t}$ can be seen as follows. Let

$$
G=\mathbb{C} \backslash \bigcup_{k=1}^{N}\left(C_{k} \cup \pi\left(C_{k}\right)\right) \backslash(\gamma[0, t] \cup \pi(\gamma[0, t])),
$$

where $\pi$ is the mirror reflection map with respect to $\partial \mathbb{H}$. If $g_{t}$ satisfies the abovementioned properties, then it sends $\partial \mathbb{H} \backslash\{\gamma(0)\}$ onto $\partial \mathbb{H}$ minus a compact interval homeomorphically so that $g_{t}$ can be extended by the Schwarz reflection to a conformal map $\widetilde{g}_{t}$ from $G$ onto $\mathbb{C} \backslash \bigcup_{i=1}^{2 N+1} \ell_{i}$ for some mutually disjoint horizontal line segments $\left\{\ell_{i}\right\}$ satisfying the condition (6.7) for some complex $a_{t}$. As is well known (e.g. [18, Theorem IX.23]), $\widetilde{g}_{t}$ with the stated properties is unique and so is its restriction $g_{t}$ to $D \backslash \gamma(0, t]$. The existence of a map $f=\widetilde{g}_{t}$ on $G$ with the stated properties is also well known (e.g. [18, Theorem IX 22]). Since the map $\widehat{f}$ defined by $\widehat{f}(z)=\overline{f(\bar{z})}, z \in G$, has the same properties as $f$, we have $f=\widehat{f}$, and in particular $f$ sends real to real. So $\left.f\right|_{D \backslash \gamma[0, t]}$ gives the desired map $g_{t}$.

For $0<s<t<t_{\gamma}$, define

$$
g_{t, s}=g_{s} \circ g_{t}^{-1}
$$

which is a conformal map from $D_{t}$ onto $D_{s} \backslash g_{s}(\gamma[s, t])$. We can then deduce from (6.7) that

$$
g_{t, s}(z)=z+\frac{a_{s}-a_{t}}{z}+o\left(\frac{1}{|z|}\right), \quad z \rightarrow \infty .
$$

Define

$$
\xi(t)=g_{t}(\gamma(t))\left(=\lim _{z \rightarrow \gamma(t), z \in D \backslash \gamma[0, t]} g_{t}(z)\right) .
$$

By the well-known boundary correspondence for $g_{t}$ (cf. [6]), $\xi(t) \in \partial \mathbb{H}$. Furthermore, by that for $g_{t, s}$, there exist unique points $\beta_{0}(t, s) \in \partial \mathbb{H}$ and $\beta_{1}(t, s) \in \partial \mathbb{H}$ such that

$$
\beta_{0}(t, s)<\xi(t)<\beta_{1}(t, s), \quad g_{t, s}\left(\beta_{0}(t, s)\right)=g_{t, s}\left(\beta_{1}(t, s)\right)=\xi(s),
$$

and it holds that

$$
\Im g_{t, s}(x+i 0+)\left\{\begin{array}{ccc}
= & 0 & x \in \partial \mathbb{H} \backslash\left(\beta_{0}(t, s), \beta_{1}(t, s)\right), \\
> & 0 & x \in\left(\beta_{0}(t, s), \beta_{1}(t, s)\right) .
\end{array}\right.
$$

We let

$$
D_{t}=\mathbb{H} \backslash \bigcup_{k=1}^{N} C_{t, k}, \quad \ell_{t, s}=\left[\beta_{0}(t, s), \beta_{1}(t, s)\right](\subset \partial \mathbb{H}) .
$$

Because of (6.12), $g_{t, s}$ extends by the Schwarz reflection to an analytic function on

$$
\mathbb{C} \backslash \Gamma_{t}, \quad \text { where } \quad \Gamma_{t}=\bigcup_{k=1}^{N}\left(C_{t, k} \cup \pi\left(C_{t, k}\right)\right) \cup \ell_{t, s} .
$$

In what follows, we may and will assume that $0 \notin \ell_{t, s}$. 
Let $\widetilde{\Gamma}_{t}=\bigcup_{k=1}^{N}\left(\widetilde{C}_{t, k} \cup \widetilde{\pi\left(C_{t, k}\right)}\right) \cup \widetilde{\ell}_{t, s}$ be the image of $\Gamma_{t}$ under the inversion $w=$ $1 / z$. Then $g_{t, s}(1 / w)$ is analytic on $\mathbb{C} \backslash \widetilde{\Gamma}_{t} \backslash\{\mathbf{0}\}$, and so is $f(w)=\left(g_{t, s}(1 / w)-\frac{1}{w}\right) / w$. Since (6.9) implies

$$
\lim _{w \rightarrow 0} f(w)=\lim _{z \rightarrow \infty} z\left\{g_{t, s}(z)-z\right\}=a_{s}-a_{t},
$$

$\mathbf{0}$ is a removable singularity of $f$ and $f$ extends to an analytic function on $\mathbb{C} \backslash \widetilde{\Gamma}_{t}$ with

$$
\mathbf{0} \in \mathbb{C} \backslash \widetilde{\Gamma}_{t}, \quad f(\mathbf{0})=a_{s}-a_{t} .
$$

We note that $\lim _{\zeta \rightarrow \infty} f(\zeta)=\lim _{z \rightarrow 0} z\left\{g_{t, s}(z)-z\right\}=0$ and so

$$
\lim _{R \rightarrow \infty} \int_{|\zeta|=R} \frac{f(\zeta)}{\zeta} d \zeta=0 .
$$

Therefore we have from (6.14) and Cauchy's integral formula

$$
a_{s}-a_{t}=\frac{1}{2 \pi i} \int_{\bigcup_{k=1}^{2 N+1} \widetilde{\gamma}_{k}} \frac{f(\zeta)}{\zeta} d \zeta,
$$

where $\tilde{\gamma}_{1}, \cdots, \widetilde{\gamma}_{N}$ (resp. $\tilde{\gamma}_{N+1}, \cdots, \widetilde{\gamma}_{2 N}$ ) are analytic contours surrounding $\widetilde{C}_{t, 1}, \cdots, \widetilde{C}_{t, N}$ (resp. $\left.\widetilde{\pi\left(C_{t, 1}\right)}, \cdots, \widetilde{\pi\left(C_{t, N}\right)}\right)$ and $\widetilde{\gamma}_{2 N+1}$ is an analytic contour surrounding $\widetilde{\ell_{t, s}}$. They are disjoint and of clockwise orientation, and $\mathbf{0}$ is located outside all of them.

Lemma 6.2. It holds for $0 \leq s<t \leq t_{\gamma}$ that

$$
a_{t}-a_{s}=\frac{1}{\pi} \int_{\beta_{0}(t, s)}^{\beta_{1}(t, s)} \Im g_{t, s}(x+i 0+) d x .
$$

Proof. From (6.15) and a change of variables of the line integral,

$$
\begin{aligned}
a_{s}-a_{t} & =\frac{1}{2 \pi i} \int_{\bigcup_{k=1}^{2 N+1} \widetilde{\gamma}_{k}} \frac{g_{t, s}\left(\frac{1}{\zeta}\right)-\frac{1}{\zeta}}{\zeta^{2}} d \zeta \\
& =-\frac{1}{2 \pi i} \int_{\bigcup_{k=1}^{2 N+1} \gamma_{k}}\left(g_{t, s}(\eta)-\eta\right) d \eta
\end{aligned}
$$

where $\gamma_{k}$ is the image of $\widetilde{\gamma}_{k}$ under $\eta=\frac{1}{\zeta}$ for $1 \leq k \leq 2 N+1$. Hence $\gamma_{1}, \cdots, \gamma_{N}$ (resp. $\left.\gamma_{N+1}, \cdots, \gamma_{2 N}\right)$ are analytic contours surrounding $C_{t, 1}, \cdots, C_{t, N}\left(\operatorname{resp} . \pi\left(C_{t, 1}\right), \cdots\right.$, $\left.\pi\left(C_{t, N}\right)\right)$ and $\gamma_{2 N+1}$ is an analytic contour surrounding $\ell_{t, s}$, all oriented clockwise.

Since $\Im g_{t, s}(\eta)$ is constant on $C_{t, k}, g_{t, s}(\eta)$ admits analytic extensions across $C_{t, k}$ from both sides. Hence the integral $\int_{\gamma_{k}}\left(g_{t, s}(\eta)-\eta\right) d \eta$ equals $\int_{C_{t, k}}\left(g_{t, s}(\eta)-\eta\right) d \eta$ for $1 \leq k \leq N$ and $\int_{\pi\left(C_{t, k}\right)}\left(g_{t, s}(\eta)-\eta\right) d \eta$ for $N+1 \leq k \leq 2 N$, the integral on $C_{t, k}$ (and on $\pi\left(C_{t, k}\right)$ ) being understood to be the sum of the integral along its upper side and lower side with clockwise orientation. Taking as $\gamma_{2 N+1}$ a rectangle with width $2 \varepsilon$ surrounding $\ell_{t, s}$, we then have

$$
\begin{aligned}
a_{s}-a_{t}= & -\frac{1}{2 \pi i} \sum_{k=1}^{N} \int_{C_{k} \cup \pi\left(C_{k}\right)}\left(g_{t, s}(\eta)-\eta\right) d \eta \\
& -\lim _{\varepsilon \downarrow 0} \frac{1}{2 \pi i} \int_{\beta_{0}(t, s)}^{\beta_{1}(t, s)}\left(\left(g_{t, s}(x+i \varepsilon)-x-i \varepsilon\right)-\left(\overline{g_{t, s}(x+i \varepsilon)}-x+i \varepsilon\right)\right) d x .
\end{aligned}
$$


Since $a_{s}-a_{t}$ is real, we conclude that $a_{s}-a_{t}$ equals

$$
-\frac{1}{2 \pi} \sum_{k=1}^{N} \int_{C_{k} \cup \pi\left(C_{k}\right)} \Im\left(g_{t, s}(\eta)-\eta\right) d \eta-\frac{1}{\pi} \int_{\beta_{0}(t, s)}^{\beta_{1}(t, s)} \Im g_{t, s}(x+i 0+) d x .
$$

But $\Im\left(g_{t, s}(\eta)-\eta\right)$ takes the same constant value from both sides of each $C_{k}$ and of each $\pi\left(C_{k}\right)$ so that the sum in the above vanishes. This establishes (6.16).

Corollary 6.3. $a_{t}$ is a strictly increasing left-continuous function in $t>0$ with $a_{0}=0$.

Proof. $a_{t}$ is strictly increasing in view of (6.12) and (6.16). Denote by $\gamma_{s}^{+}, \gamma_{s}^{-}$the points of 'both sides' of the arc $\gamma$ corresponding to $\gamma_{s}$. As $s \uparrow t, \gamma_{s}^{-} \rightarrow \gamma_{t}$ (resp. $\left.\gamma_{s}^{+} \rightarrow \gamma_{t}\right)$ so that $\beta_{0}(t, s)=g_{t}\left(\gamma_{s}^{-}\right) \uparrow g_{t}\left(\gamma_{t}\right)=\xi_{t}\left(\operatorname{resp} . \beta_{1}(t, s)=g_{t}\left(\gamma_{s}^{+}\right) \downarrow g_{t}\left(\gamma_{t}\right)=\right.$ $\left.\xi_{t}\right) . a_{t}$ is thus left continuous. Since $g_{0}(z)=z, z \in D$, due to the uniqueness of $g_{0}$ and (6.7), we have $a_{0}=0$.

6.3. Chordal Komatu-Loewner differential equation. Keeping the setting of the preceding subsection, we now derive the Komatu-Loewner differential equation (1.3), but with its left-hand side being replaced by the left derivative $\frac{\partial^{-} g_{t}(z)}{\partial a_{t}}$ with respect to the strictly increasing left continuous function $a_{t}$ studied in the preceding subsection.

Let $Z^{t, *}$ be the BMD on the standard slit domain $D_{t}$ and $\Psi_{t}(z, \zeta), z \in D_{t}, \zeta \in$ $\partial \mathbb{H}$, be the complex Poisson kernel of $Z^{t, *}$, namely, an analytic function on $D_{t}$ with its imaginary part being the Poisson kernel $K_{t}^{*}(z, \zeta)$ of $Z^{t, *}$ subject to the normalization (6.1).

Theorem 6.4. The map $g_{t}$ satisfies the following chordal Komatu-Loewner differential equation: for $t \in\left(0, t_{\gamma}\right]$ and $z \in D \backslash \gamma[0, t], g_{t}(z)$ is left differentiable with respect to $a_{t}$ and

$$
\frac{\partial^{-} g_{t}(z)}{\partial a_{t}}=-\pi \Psi_{t}\left(g_{t}(z), \xi(t)\right), \quad g_{0}(z)=z .
$$

Proof. We proceed along the same line as in the proof of Theorem 3.1 in Bauer and Friedrich [3], but with several modifications stated in $\S 1$.

We consider the analytic function

$$
F(z)=g_{t, s}(z)-z, \quad z \in D_{t},
$$

which satisfies

$$
F(z)=\frac{a_{s}-a_{t}}{z}+o(1 /|z|)
$$

Then, $f(z):=\Im F(z)$ is harmonic on $D_{t}$ and takes a constant, say $f_{i}$ on each slit $C_{t, i}$. As $\lim _{z \rightarrow \infty} f(z)=0$ by (6.18), the formula (4.21) holds: for $z \in D_{t}$,

$$
f(z)=\sum_{k=1}^{N} f_{k} \varphi_{t}^{(k)}(z)-\frac{1}{2} \int_{\partial \mathbb{H}} \frac{\partial G_{t}^{0}(z, \zeta)}{\partial \mathbf{n}_{\zeta}} f(\zeta) d s(\zeta)
$$

where $\left\{\varphi_{t}^{(i)}\right\}$ is the harmonic basis and $G_{t}^{0}(z, \zeta)$ is the Green function of the ABM on the domain $D_{t}=\mathbb{H} \backslash \bigcup_{k=1}^{N} C_{t, k}$. 
Since $f$ is the imaginary part of the analytic function $F$, its period around $C_{t, i}$ vanishes, and we have from 4.22)

$$
0=-\sum_{k=1}^{N} f_{k} a_{t, k i}-\int_{\partial \mathbb{H}} \frac{\partial \varphi_{t}^{(i)}(\zeta)}{\partial \mathbf{n}_{\zeta}} f(\zeta) d s(\zeta),
$$

for every $1 \leq i \leq N$, where $a_{t, k i}$ denotes the period of $\varphi_{t}^{(i)}$ around the slit $C_{t, k}$.

Denote by $\mathcal{A}_{t}$ the symmetric matrix with $(i, j)$-component $a_{t, i j}$ and by $q_{t, i j}$ the $(i, j)$-component of $\mathcal{A}_{t}^{-1}$. Multiply both sides of (6.20) by $\sum_{j=1}^{N} \varphi_{t}^{(j)}(z) q_{t, j i}$ and adding up in $i$, and finally add the resulting identity to (6.19). We are then left with

$$
f(z)=\int_{\partial \mathbb{H}} K_{t}^{*}(z, \zeta) f(\zeta) d s(\zeta)
$$

in view of the expression (5.2) of the Poisson kernel $K_{s}^{*}(z, \zeta)$ of $Z^{t, *}$. Since $f$ vanishes on $\partial \mathbb{H} \backslash\left[\beta_{0}(t, s), \beta_{1}(t, s)\right]$, we have

$$
f(z)=\int_{\beta_{0}(t, s)}^{\beta_{1}(t, s)} K_{t}^{*}(z, x) f(x) d x,
$$

and accordingly,

$$
g_{t, s}(z)-z=\int_{\beta_{0}(t, s)}^{\beta_{1}(t, s)} \Psi_{t}(z, x) \Im g_{t, s}(x) d x+c,
$$

for some real constant $c$. By taking the normalization (6.8) and Lemma 6.1 into account, we let $z \rightarrow \infty$ in (6.21) to get $c=0$. We then substitute $z=g_{t}(w)$ to obtain

$$
g_{s}(z)-g_{t}(z)=\int_{\beta_{0}(t, s)}^{\beta_{1}(t, s)} \Psi_{t}\left(g_{t}(z), x\right) \Im g_{t, s}(x) d x .
$$

By Lemma 6.1 (ii), $\Psi_{t}(z, \zeta)=u_{t}(z, \zeta)+i K_{t}^{*}(z, \zeta)$ is continuous in $\zeta \in \partial \mathbb{H}$ for each $z \in D$, and consequently (6.16), (6.22) and the mean value theorem of integration imply that, for some $x^{\prime}, x^{\prime \prime} \in\left(\beta_{0}(t, s), \beta_{1}(t, s)\right)$,

$$
\frac{g_{s}(z)-g_{t}(z)}{a_{s}-a_{t}}=-\pi u_{t}\left(g_{t}(z), x^{\prime}\right)-i \pi K_{t}^{*}\left(g_{t}(z), x^{\prime \prime}\right) .
$$

If we let $s \uparrow t$, then both $\beta_{0}(t, s)$ and $\beta_{1}(t, s)$ converge to $\xi(t)$ as was observed in the proof of Corollary 6.3, and we arrive at the desired equation (6.17).

\section{A probabilistic Representation of $\Im g_{t}$}

For the conformal map $g_{t}$ studied in the preceding two subsections, we shall give in this section a probabilistic representation of $\Im g_{t}$ in terms of the absorbing Brownian motion (ABM in abbreviation) on $\mathbb{H}$ and the BMD on $\left(\mathbb{H} \backslash \bigcup_{i=1}^{N} C_{i}\right) \cup$ $\left\{c_{1}^{*}, \cdots, c_{N}^{*}\right\}$. This representation will readily yield basic properties of the family $\left\{\Im g_{t}\right\}$ that will be utilized in the next section.

More generally we start with an $(N+1)$-connected domain (see $\S 3.3)$

$$
D=\mathbb{H} \backslash K \quad \text { with } \quad K=\bigcup_{i=1}^{N} A_{i} .
$$

We call a set $F \subset \mathbb{H}$ a compact $\mathbb{H}$-hull if $\bar{F}$ is compact, $F=\bar{F} \cap \mathbb{H}$ and $\mathbb{H} \backslash F$ is simply connected. We consider a compact $\mathbb{H}$-hull $F$ satisfying $F \subset D$. Denote by 
$Z^{\mathbb{H}}=\left(Z_{t}^{\mathbb{H}}, \mathbb{P}_{z}^{\mathbb{H}}\right)$ the ABM on $\mathbb{H}$ and by $Z^{\mathbb{H}, *}=\left(Z_{t}^{\mathbb{H}, *}, \mathbb{P}_{z}^{\mathbb{H}, *}\right)$ the BMD on $D^{*}=D \cup K^{*}$ with $K^{*}=\left\{a_{1}^{*}, \cdots, a_{N}^{*}\right\}$ obtained from $Z^{\mathbb{H}}$ by regarding each compact continuum $A_{i}$ as one point $a_{i}^{*}$.

For $r>0$, let $\Gamma_{r}=\{z=x+i y: y=r\}$ and

$$
v^{*}(z):=\lim _{r \rightarrow \infty} r \cdot \mathbb{P}_{z}^{\mathbb{H H}, *}\left(\sigma_{\Gamma_{r}}<\sigma_{F}\right), \quad z \in D^{*} \backslash F .
$$

Theorem 7.1. (i) The function $v^{*}$ on $D^{*} \backslash F$ is well defined and is $Z^{*}$-harmonic on $D^{*} \backslash F$. Furthermore

$$
v^{*}(z)=v(z)+\sum_{j=1}^{N} \mathbb{P}_{z}^{\mathbb{H}}\left(\sigma_{K}<\sigma_{F}, Z_{\sigma_{K}}^{\mathbb{H}} \in A_{j}\right) v^{*}\left(a_{j}^{*}\right), z \in D \backslash F,
$$

where

$$
\begin{gathered}
v(z)=\Im z-\mathbb{E}_{z}^{\mathbb{H}}\left[\Im Z_{\sigma_{F \cup K}}^{\mathbb{H}} ; \sigma_{F \cup K}<\infty\right](\geq 0), \\
v^{*}\left(a_{i}^{*}\right)=\sum_{j=1}^{N} \frac{M_{i j}}{1-R_{j}^{*}} \int_{\eta_{j}} v(z) \nu_{j}(d z), \quad 1 \leq i \leq N .
\end{gathered}
$$

Here $\eta_{1}, \cdots, \eta_{N}$ are mutually disjoint smooth Jordan curves surrounding $A_{1}, \cdots$, $A_{N}$, respectively,

$$
\begin{gathered}
\nu_{i}(d z)=\mathbb{P}_{a_{i}^{*}}^{\mathbb{H}, *}\left(Z_{\sigma_{\eta_{i}}}^{\mathbb{H}, *} \in d z\right), \quad 1 \leq i \leq N, \\
R_{i}^{*}=\int_{\eta_{i}} \mathbb{P}_{z}^{\mathbb{H}}\left(\sigma_{K}<\sigma_{F}, Z_{\sigma_{K}}^{\mathbb{H}} \in A_{i}\right) \nu_{i}(d z), \quad 1 \leq i \leq N,
\end{gathered}
$$

and $M_{i j}$ is the $(i, j)$-entry of the matrix $M=\sum_{n=0}^{\infty}\left(Q^{*}\right)^{n}$ for a matrix $Q^{*}$ with entries

$$
q_{i j}^{*}= \begin{cases}\mathbb{P}_{a_{i}^{*}}^{\mathbb{H}, *}\left(\sigma_{K^{*}}<\sigma_{F}, Z_{\sigma^{*}}^{\mathbb{H}, *}=a_{j}^{*}\right) /\left(1-R_{i}^{*}\right) & \text { if } i \neq j, \quad 1 \leq i, j \leq N . \\ 0 & \text { if } i=j,\end{cases}
$$

(ii) $\left.v^{*}\right|_{D}$ admits a unique harmonic conjugate $u^{*}$ such that $f(z)=u^{*}(z)+$ $i v^{*}(z), z \in D$, is analytic on $D$ and

$$
f(z)=z+\frac{a}{z}+o\left(\frac{1}{z}\right), \quad z \rightarrow \infty
$$

for some positive constant a.

A proof of this theorem will be given in Appendix 1 by a series of lemmas. We remark that the way of constructing $v^{*}$ in the above theorem is due to G. Lawler [13], where ERBM on the $N$-connected domain was utilized in place of the current BMD.

Theorem 7.2. The analytic function $f$ of Theorem 7.1 is a conformal mapping from $\mathbb{H} \backslash(F \cup K)$ onto $\mathbb{H} \backslash \bigcup_{i=1}^{N} \widetilde{C}_{i}$, where $\widetilde{C}_{i}, 1 \leq i \leq N$, are mutually disjoint horizontal line segments in $\mathbb{H}$.

Proof. Since $v^{*}=0$ on $\partial(\mathbb{H} \backslash \bar{F})$, by Schwarz reflection we can extend $f$ to an analytic map from

$$
D_{1}=\overline{\mathbb{C}} \backslash(\bar{F} \cup K \cup \pi(\bar{F} \cup K))
$$


into $\overline{\mathbb{C}}$, which satisfies

$$
f(z)=z+\frac{a}{z}+o\left(\frac{1}{|z|}\right), \quad z \rightarrow \infty .
$$

Let $D_{2}=\overline{\mathbb{C}} \backslash f\left(\partial D_{1}\right)$, where $f\left(\partial D_{1}\right)$ is defined in the sense of (11.1). Since $\Im f=v^{*}\left(a_{j}^{*}\right)$ on $A_{j}$ and $\Im f=0$ on $\bar{F}$,

$$
f\left(\partial D_{1}\right) \subset\left\{z=x+i y \in \mathbb{C}: y= \pm v^{*}\left(a_{j}^{*}\right) \text { for } j=1, \cdots, N \text { or } y=0\right\} .
$$

This implies that the complement of $D_{2}$ has empty interior, and so

$$
f\left(\partial D_{1}\right)=\partial D_{2} .
$$

We next show that $\infty$ is an interior point of $D_{2}$. Since $f$ has multiplicity 1 near infinity, this will then imply by Theorem 11.2 that $f$ is a conformal mapping from $D_{1}$ to $D_{2}$. To this end, it suffices to show that $f$ maps $F$ and each $A_{j}$ (understood in the sense the limit points (11.1) ) into a bounded subset in $\mathbb{C}$.

Let $\phi$ be the conformal map of $\mathbb{H} \backslash F$ onto $\mathbb{H}$ that satisfies the hydrodynamic normalization (17.11) at infinity (but with a possibly different constant $a$ ), and set $g=f \circ \phi^{-1}$. Clearly, $g$ is well defined and analytic in $\mathbb{H}$. Let $O$ be an open neighborhood of the interval $I=\phi(F)$ (understood in the sense of limit points) that is disjoint from $\phi(K)$. The imaginary part of $g(z)$ tends to 0 as $y=\Im z \rightarrow 0$ in $O \cap \mathbb{H}$. Thus $g$ extends analytically across the real line to be an analytic function in the disk $O$. In particular, $g$ is bounded in every compact subset $O$. This in particular implies that $f(F)$ is bounded.

Similarly, for each $j=1, \ldots, N$, since $A_{j}$ is a compact continuum, there is a conformal map $\phi_{j}$ of the complement of the interval $[0,1]$ in $\mathbb{C}$ onto the complement of $A_{j}$. Let $O_{j}$ be a relatively compact open neighborhood of $A_{j}$ that is disjoint from the other boundary components. Then $\widehat{O}_{j}=\phi_{j}^{-1}\left(O_{j}\right)$ is an open neighborhood of $[0,1]$, and $g_{j}=f \circ \phi_{j}$ is analytic in $\widehat{O}_{j}$. Since $\phi_{j}(z) \rightarrow A_{j}$ as $z \rightarrow[0,1]$, the imaginary part $\Im g_{j}(z)=v^{*}\left(\phi_{j}(z) \rightarrow v^{*}\left(a_{j}^{*}\right)\right.$ as $z \rightarrow[0,1]$. By the argument similar to that in the previous paragraph, $g_{j}$ is bounded on every compact subset of $O_{i}$ and so $f=g_{j} \circ \phi_{j}^{-1}$ is bounded near $A_{j}$.

In view of the above, we conclude from (7.11) that the pre-image of $\infty \in D_{2}$ under $f$ is $\infty$ with multiplicity 1 . Theorem 11.2 together with (7.12) implies that $f$ is conformal from $D_{1}$ onto $D_{2}$. As $f$ is a topological homeomorphism between $D_{1}$ and $D_{2}, D_{2}$ has to be $(2 N+1)$-connected as well. Thus $D_{2}$ is a slit domain with $2 N+1$ disjoint horizontal line segments symmetric relative to $\partial \mathbb{H}$. None of them degenerates to a single point. In fact, if one of them reduces to a point $p$, then, due to (7.11), $p$ is not a pole or an essential singularity of the analytic function $f^{-1}$ so that $p$ is removable, contradicting the assumption that the boundary components of $D_{1}$ are continua.

Let us return to the setting in the last two sections: $D=\mathbb{H} \backslash K$ is a standard slit domain with $K=\bigcup_{i=1}^{N} C_{i}$ and $\gamma=\left\{\gamma(t), 0 \leq t \leq t_{\gamma}\right\}$ is a Jordan arc satisfying condition (6.6). For each fixed $t \in\left[0, t_{\gamma}\right]$, let $g_{t}$ be the unique conformal map from $D \backslash \gamma(0, t]$ onto a standard slit domain satisfying property (6.7) at infinity. $g_{0}$ is the identity map. $Z^{\mathbb{H}, *}=\left(Z_{t}^{\mathbb{H}, *}, \mathbb{P}_{z}^{\mathbb{H}, *}\right)$ will denote the BMD on $D=D \cup K^{*}$ with $K^{*}=\left\{c_{1},{ }^{*}, \cdots, c_{N}^{*}\right\}$ obtained from the ABM $Z^{\mathbb{H}}$ by regarding each slit $C_{i}$ as a point $c_{i}^{*}$. 
For each $t \in\left[0, t_{\gamma}\right]$, we let $F_{t}=\gamma[0, t]$. The functions $v^{*}, v$ and the quantities $R_{i}^{*}, q_{i j}^{*}$ specified by Theorem 7.1 but for $C_{i}, c_{i}^{*}, 1 \leq i \leq N$, and $F_{t}$ in place of $A_{i}, a_{i}^{*}, 1 \leq i \leq N$, and $F$ will be designated by $v_{t}^{*}, v_{t}$ and $R_{i}^{*}(t), q_{i j}^{*}(t)$, respectively.

By virtue of Theorem 7.2 it then holds that

$$
v_{t}^{*}(z)=\Im g_{t}(z), \quad z \in D \backslash F_{t} .
$$

Proposition 7.3. The function $v_{t}^{*}(z)$ can be continuously extended to $K \cup \partial \mathbb{H} \backslash$ $\{\gamma(0)\}$ as a jointly continuous function in $[0, s] \times(\overline{\mathbb{H}} \backslash \gamma[0, s])$ for each $s \in\left(0, t_{\gamma}\right]$. Moreover,

$$
0<\inf _{t \in\left[0, t_{\gamma}\right], 1 \leq k \leq N} v_{t}^{*}\left(c_{k}^{*}\right) \leq \sup _{t \in\left[0, t_{\gamma}\right], 1 \leq k \leq N} v_{t}^{*}\left(c_{k}^{*}\right)<\infty
$$

and for every $\varepsilon>0$,

$$
\lim _{t \downarrow 0} v_{t}^{*}(z)=v_{0}^{*}(z)=\Im z, \quad z \in \overline{\mathbb{H}} \backslash \gamma[0, \varepsilon] .
$$

Proof. Since any one-point set is polar with respect to the planar Brownian motion, we have by the continuity of Brownian motion and the curve $\gamma$ that for every $z \in \mathbb{H}$,

$$
\mathbb{P}_{z}^{\mathbb{H}}\left(\lim _{s \rightarrow t} \sigma_{F_{s}}=\sigma_{F_{t}}\right)=1, \quad \mathbb{P}_{z}^{\mathbb{H}}\left(\lim _{s \rightarrow t} \sigma_{F_{s} \cup K}=\sigma_{F_{t} \cup K}\right)=1 .
$$

Similarly it holds that

$$
\mathbb{P}_{z}^{\mathbb{H}, *}\left(\lim _{s \rightarrow t} \sigma_{F_{s}}=\sigma_{F_{t}}\right)=1 \quad \text { for every } z \in D^{*} .
$$

(7.17) implies that $h(z, t)=\mathbb{E}_{z}^{\mathbb{H}}\left[\Im Z_{\sigma_{F_{t}} \cup K}^{\mathbb{H}}, \sigma_{F_{t} \cup K}<\infty\right]$ is continuous in $t \in[0, s]$ for each $z \in \mathbb{H} \backslash \gamma[0, s]$. Since $h(z, t)$ is harmonic in $z \in \mathbb{H} \backslash \gamma[0, s]$ and by taking a constant value on each slit $C_{i}$, we get the joint continuity of $v_{t}(z)$ in $(t, z) \in$ $[0, s] \times(\overline{\mathbb{H}} \backslash \gamma[0, s])$.

On the other hand, in view of (7.18),$R_{i}^{*}(t)$ is a decreasing continuous function in $t$. For each $1 \leq i \leq N$,

$$
\sum_{j: j \neq i} q_{i j}^{*}(t)=\frac{\mathbb{P}_{c_{i}^{*}}^{\mathbb{H}, *}\left(\sigma_{K^{*}}<\sigma_{F_{t}}, Z_{\sigma_{K^{*}}}^{\mathbb{H}, *} \neq c_{i}^{*}\right)}{1-R_{i}^{*}(t)},
$$

which by (7.18) is continuous in $t$, and so

$$
\lambda:=\max _{1 \leq i \leq N, 0 \leq t \leq t_{\gamma}} \sum_{j: j \neq i} q_{i j}^{*}(t)<1 .
$$

It follows that $M_{i j}(t) \leq 1+1 /(1-\lambda) \leq 2 /(1-\lambda)$ for every $t \in\left[0, t_{\gamma}\right]$ and that $M_{i j}(t)$ is continuous in $t$. One concludes from (7.5) and the joint continuity of $v_{t}(z)$ that $v_{t}^{*}\left(c_{i}^{*}\right)$ is a continuous function in $t$ for every $1 \leq i \leq N$. This and (17.3) yield the joint continuity of $v_{t}^{*}(z)$ in $(t, z) \in[0, s] \times(\overline{\mathbb{H}} \backslash \gamma[0, s])$, as well as (7.15) and (7.16).

In the remainder of this paper, for a Borel set $A \subset \overline{\mathbb{H}}$, we use $\partial_{p} A$ to denote the boundary of $A$ with respect to the topology induced by the path distance in $\mathbb{H} \backslash A$. For instance, when $A(\subset \mathbb{H})$ is a horizontal line segment, $\partial_{p} A$ consists of the upper part $A^{+}$and the lower part $A^{-}$of the line segment $A$. 
Theorem 7.4. For each $t>0$, the conformal map $g_{t}(z)$ extends continuously to $\partial_{p} K$. Moreover, $\left\{g_{s}(z) ; s \in[0, t]\right\}$ are locally equi-continuous and locally uniformly bounded in $z \in D \cup \partial_{p} K \cup \partial_{p}(\mathbb{H} \backslash \gamma[0, t])$.

Proof. Let $\widehat{F}_{t}=\gamma[0, t] \cup \pi(\gamma[0, t])$, where, as before, $\pi$ denotes the mirror reflection with respect to $\partial \mathbb{H}$. By the Schwarz reflection principle, for each $t>0$, we can extend $g_{t}(z)$ to be an analytic function in $\mathbb{C} \backslash\left(K \cup \pi(K) \cup \widehat{F}_{t}\right)$. Let $u_{t}^{*}(z)=\Re g_{t}(z)$ and $v_{t}^{*}(z)=\Im g_{t}(z)$. We note that, owing to Proposition [7.3, $v_{s}^{*}(z)$ is continuously extendable in $z$ to $\mathbb{C} \backslash \widehat{F}_{t}$ to be jointly continuous in $(s, z) \in[0, t] \times\left(\mathbb{C} \backslash \widehat{F}_{t}\right)$.

Since for each $s \geq 0, v_{s}^{*}(z)$ is harmonic in $z \in \mathbb{C} \backslash\left(K \cup \pi(K) \cup \widehat{F}_{t}\right)$, it follows from the integral representation for harmonic functions in disks in terms of Poisson kernels that $\nabla_{z} v_{s}^{*}(z)$ is jointly continuous in $(s, z) \in[0, t] \times\left(\mathbb{C} \backslash\left(K \cup \pi(K) \cup \widehat{F}_{t}\right)\right)$. Hence by the Cauchy-Riemann equation, so is $\nabla_{z} u_{s}^{*}(z)$. This implies that $\left\{u_{s}^{*}(z)\right.$; $s \in[0, t]\}$ are locally equi-continuous in $z \in \mathbb{C} \backslash\left(K \cup \pi(K) \cup \widehat{F}_{t}\right)$. On the other hand, it follows from (6.23) that for each fixed $z \in D, t \mapsto u_{t}^{*}(z)$ is bounded in $t \in\left(0, t_{\gamma}\right]$. These together with Proposition 7.3 yield that $\left\{g_{s}(z) ; s \in[0, t]\right\}$ are locally equi-continuous and locally uniformly bounded in $z \in D \cup \partial \mathbb{H} \backslash \gamma[0, t]$.

We next show that $\left\{g_{s}(z) ; s \in[0, t]\right\}$ are in fact locally equi-continuous and locally uniformly bounded in $z \in D \cup \partial_{p} K \cup \partial_{P}(\mathbb{H} \backslash \gamma[0, t])$. Since $\Im g_{s}(z)$ takes constant value on each slit $\partial C_{j}$, by Schwarz reflection principle, $g_{s}(z)=u_{s}^{*}(z)+$ $i v_{s}^{*}(z)$ can be extended to be an analytic function across $C_{j}^{+}$(resp. $C_{j}^{-}$) except at the two endpoints of $C_{j}$. As the harmonic function $v_{s}^{*}(z)$ is jointly continuous in $(s, z)$, the same argument as above applies, and we conclude that $\left\{g_{s}(z) ; s \in[0, t]\right\}$ are locally equi-continuous and locally uniformly bounded in a neighborhood of every point in $C_{j}^{+} \cup C_{j}^{-}$with the two endpoints removed.

For the left endpoint $z_{1}$ of the slit $C_{j}$, take $\varepsilon>0$ small so that it is less than one-half of the length of $C_{j}$ and that $B\left(z_{1}, \varepsilon\right) \backslash C_{j} \subset D$. Then $\psi(z)=\left(z-z_{1}\right)^{1 / 2}$ maps $B\left(z_{1}, \varepsilon\right) \backslash C_{j}$ conformally onto $B(0, \sqrt{\varepsilon}) \cap \mathbb{H}$. Consequently, $g_{s} \circ \psi^{-1}(z)=$ $g_{s}\left(z^{2}+z_{1}\right)$ is an analytic function in $z \in B(0, \sqrt{\varepsilon}) \cap \mathbb{H}$ that is continuous up to $B(0, \sqrt{\varepsilon}) \cap \partial \mathbb{H} \backslash\{0\}$ and $v_{s}^{*}\left(z^{2}+z_{1}\right)=\Im g_{s}\left(z^{2}+z_{1}\right)$ takes constant value there. By the Schwarz reflection, $g_{s}\left(z^{2}+z_{1}\right)$ extends to $B(0, \sqrt{\varepsilon}) \backslash\{0\}$. Thus 0 is an isolated singularity of $g_{s}\left(z^{2}+z_{1}\right)$. Since $\Im g_{s}\left(z^{2}+z_{1}\right)$ is bounded near the origin, it has to be a removable singularity. It follows that $g_{s}\left(z^{2}+z_{1}\right)$ can be extended to be analytic in $z \in B(0, \sqrt{\varepsilon})$ and $\Im g_{s}\left(z^{2}+z_{1}\right)=v_{s}^{*}\left(z^{2}+z_{1}\right)$ is jointly continuous in $(s, z)$. Thus the same argument as above is applicable again in concluding that $\left\{g_{s}(z) ; s \in[0, t]\right\}$ are equi-continuous and uniformly bounded in $B\left(z_{1}, \varepsilon / 2\right) \cap\left(D \cup \partial_{p} K\right)$. The case for the right endpoint of $C_{j}$ can be dealt with analogously.

The same argument with trivial modification establishes the analogous assertion with $\gamma[0, t]$ in place of $C_{j}$. By an obvious covering argument, we arrive at the desired conclusion of the theorem.

We will show in Theorem 8.3 below that for every $t \in\left(0, t_{\gamma}\right], g_{s}(z)$ is in fact jointly continuous in $(s, z) \in[0, t] \times\left(D \cup \partial_{p} K \cup \partial \mathbb{H} \backslash \gamma[0, t]\right)$.

\section{Continuity of $g_{t}, a_{t}, D_{t} \operatorname{AnD} \xi(t)$}

Throughout this section, we maintain the setting of $\S 6.2$ and $\S 6.3$. Let $g_{t}(z)$ be the conformal map from $D \backslash \gamma(0, t]$ onto a standard slit domain $D_{t}$ satisfying the hydrodynamic normalization condition (6.7) at infinity. The goal of this section is to show, using Proposition 7.3 and Theorem 7.4 , the continuity of $g_{t}(z)$ in $t$ with 
certain uniformity in $z$, and thereby derive the continuity of the function $a_{t}$ in (6.7), the standard slit domain $D_{t}$ and the position $\xi(t) \in \partial \mathbb{H}$ defined in (6.10).

Recall the conformal map $g_{t, s}=g_{s} \circ g_{t}^{-1}$ defined in $\S 6.2$ for $0 \leq s<t<t_{\gamma}$, which sends $D_{t}$ onto $D_{s} \backslash g_{s}(\gamma[s, t])$. Denote by $C_{t, i}^{+}$(resp. $C_{t, i}^{-}$) the upper (resp. lower) side of the slit $C_{t, i}$, and $\partial_{p} K_{t}:=\bigcup_{j=1}^{N}\left(C_{t, i}^{+} \cup C_{t, i}^{-}\right)$. In the proof of the next proposition, we will use the following identity that follows from (6.21) with $c=0$, Lemma 6.1 and Theorem 7.4 .

$$
g_{t, s}(z)-z=\int_{\ell_{t, s}} \Psi_{t}(z, x) \Im g_{t, s}(x) d x \quad \text { for } s<t \text { and } z \in D_{t} \cup \partial_{p} K_{t} \cup \partial \mathbb{H} .
$$

Here $\ell_{t, s}$ denotes the interval $\left(\beta_{0}(t, s), \beta_{1}(t, s)\right)(\subset \partial \mathbb{H})$.

Theorem 8.1. For a fixed $t \in\left(0, t_{\gamma}\right], \lim _{s \uparrow t} g_{t, s}(z)=z$ uniformly in $z$ on each compact subset of $D_{t} \cup \partial_{p} K_{t} \cup(\partial \mathbb{H} \backslash\{\xi(t)\})$.

Proof. We let $M_{\gamma}=\sup _{t \in\left[0, t_{\gamma}\right]} \Im \gamma(t)$. We have by (7.3), (7.4) and (7.15),

$$
\begin{aligned}
& \sup _{0 \leq s<t} \sup _{x \in \ell_{t, s}} \Im g_{t, s}(x)=\sup _{0 \leq s<t} \sup _{s \leq s^{\prime} \leq t} \Im g_{s}\left(\gamma\left(s^{\prime}\right)\right)=\sup _{0 \leq s^{\prime} \leq t} \sup _{0 \leq s \leq s^{\prime}} v_{s}^{*}\left(\gamma\left(s^{\prime}\right)\right) \\
& (8.2) \leq M_{\gamma}+\sum_{j=1}^{N} \sup _{0 \leq s \leq t_{\gamma}} v_{s}^{*}\left(c_{j}^{*}\right)=: M_{1}<\infty .
\end{aligned}
$$

For any compact subset $L$ of $D_{t} \cup \partial_{p} K_{t} \cup(\partial \mathbb{H} \backslash\{\xi(t)\})$, choose $\varepsilon>0$ and $\delta>0$ such that $L \cap B_{\varepsilon}(\xi(t))=\emptyset$ and $\ell_{t, t-\delta} \subset B_{\varepsilon}(\xi(t))$. We then see from Lemma 6.1(ii) that $M_{2}=\sup \left\{\left|\Psi_{t}(z, \zeta)\right|: z \in L, \zeta \in \ell_{t, t-\delta}\right\}$ is finite. Hence (8.1) implies that, for any $s \in(t-\delta, t), \sup _{z \in L}\left|g_{t, s}(z)-z\right| \leq M_{1} M_{2}\left|\ell_{t, s}\right|<2 M_{1} M_{2} \varepsilon$.

The inverse $g_{t, s}^{-1}=g_{t} \circ g_{s}^{-1}$ of $g_{t, s}$ is a conformal map from $D_{s} \backslash g_{s}(\gamma[s, t])$ onto the standard slit domain $D_{t}$ satisfying

$$
g_{t, s}^{-1}(z)=z+\frac{a_{t}-a_{s}}{z}+o\left(\frac{1}{|z|}\right), \quad \text { as }|z| \rightarrow \infty, \quad 0 \leq s<t \leq t_{\gamma} .
$$

By the Schwarz reflection, $g_{t, s}^{-1}, 0 \leq s<t \leq t_{\gamma}$, extends to a conformal map from $\mathbb{C} \backslash \Lambda_{s}$ onto $\mathbb{C} \backslash \Gamma_{t}$ still satisfying (8.3), where

$$
\Lambda_{s}=\bigcup_{k=1}^{N}\left(C_{s, k} \cup \pi\left(C_{s, k}\right)\right) \cup g_{s}(\gamma[s, t]) \cup \pi\left(g_{s}(\gamma[s, t])\right), \Gamma_{t}=\bigcup_{k=1}^{N}\left(C_{t, k} \cup \pi\left(C_{t, k}\right)\right) \cup \ell_{t, s} .
$$

Theorem 8.2. For a fixed $s \in\left[0, t_{\gamma}\right), \lim _{t \downarrow s} g_{t, s}^{-1}(z)=z$ uniformly in $z$ on each compact subset of $D_{s} \cup \partial_{p} K_{s} \cup(\partial \mathbb{H} \backslash\{\xi(s)\})$.

Proof. Without loss of generality, we may assume that $s=0$, and so $g_{t, s}^{-1}=g_{t}$. For any $0<\varepsilon<R$ with $\overline{B(\gamma(0), \varepsilon)} \cap K=\emptyset, K \subset B(\gamma(0), R)$, let $A_{\varepsilon, R}=$ $((\overline{\mathbb{H}} \backslash K) \cap(B(\gamma(0), R) \backslash B(\gamma(0), \varepsilon))) \cup \partial_{p} K$ and take $\delta>0$ such that $\gamma[0, \delta] \subset$ $B(\gamma(0), \varepsilon)$. In view of Theorem 7.4, the family $\left\{g_{t}(z)=u_{t}^{*}(z)+i v_{t}^{*}(z) ; 0 \leq t \leq \delta\right\}$ is uniformly bounded and equi-continuous in $z \in A_{\varepsilon, R}$. Therefore, every sequence $t_{n} \downarrow 0$ admits a subsequence still denoted as $t_{n}$ such that $\widetilde{g}_{t_{n}}(z):=g_{t_{n}}(z)-z$ converges uniformly on $A_{\varepsilon, R}$, as $n \rightarrow \infty$, to a function $f$, which is analytic on $D \cap(B(\gamma(0), R) \backslash \overline{B(\gamma(0), \varepsilon)})$. It follows from (7.16) that $\Im f(z)=0$ and so $f$ is a constant. 
To show this constant is zero, extend $g_{t}$ by the Schwarz reflection as before. Since $g_{t}$ is a conformal map from $\overline{\mathbb{C}} \backslash \overline{B(\gamma(0), R)}$ to the outside of $g_{t}(\partial \overline{B(\gamma(0), R)})$, it easily follows that $g_{t}(z)-z$ also converges uniformly (with respect to the spherical metric) on $\overline{\mathbb{C}} \backslash \overline{B(\gamma(0), R)}$ to an analytic function that extends $f$. Near $\infty$ the Laurent series of $g_{t}$ begins with $g_{t}(z)=z+O(1 / z)$ so that $f(z) \rightarrow 0$ as $z \rightarrow \infty$.

Theorems 8.1 8.2 together with Theorem 7.4 immediately yield the following.

Theorem 8.3. For every $0<s \leq t_{\gamma}, g_{t}(z)$ is jointly continuous in $(t, z) \in[0, s] \times$ $\left(\left(D \times \partial_{p} K \cup \partial \mathbb{H}\right) \backslash \gamma[0, s]\right)$.

Theorem 8.2 in particular implies that, for a fixed $s \in\left[0, t_{\gamma}\right]$,

$$
\lim _{t \downarrow s} g_{t, s}^{-1}(z)=z \text { uniformly on each compact subset of } \mathbb{C} \backslash \Lambda_{s}^{0},
$$

for $\Lambda_{s}^{0}=\bigcup_{k=1}^{N}\left(C_{s, k} \cup \pi\left(C_{s, k}\right)\right) \cup\{\xi(s)\}$. This immediately leads us to the right continuity of $a_{t}$ in the following manner.

We may assume that $\xi(s) \neq \mathbf{0}$. Let $\widetilde{\Lambda}_{s}^{0}=\bigcup_{k=1}^{N}\left(\widetilde{C_{s, k}} \cup \widetilde{\pi\left(C_{s, k}\right)}\right) \cup \widetilde{\{\xi(s)\}}$ be the image of $\Lambda_{s}^{0}$ under the inversion $w=\frac{1}{z}$. Then $h_{t, s}(w)=\frac{1}{w}\left(g_{t, s}^{-1}\left(\frac{1}{w}\right)-\frac{1}{w}\right)$ is analytic on $\mathbb{C} \backslash \widetilde{\Lambda}_{s}^{0} \backslash\{\mathbf{0}\}$. Just as (6.15), we can then get from (8.3) the integral formula

$$
a_{t}-a_{s}=\frac{1}{2 \pi i} \int_{\bigcup_{k=1}^{2 N+1} \widetilde{\gamma}_{k}} \frac{h_{t, s}(\zeta)}{\zeta} d \zeta, \quad s<t,
$$

where $\widetilde{\gamma}_{1}, \cdots, \widetilde{\gamma}_{N}$ (resp. $\left.\widetilde{\gamma}_{N+1}, \cdots, \widetilde{\gamma}_{2 N}\right)$ are analytic contours surrounding $\widetilde{C_{s, 1}}, \cdots$, $\widetilde{C_{s, N}}\left(\operatorname{resp} . \widetilde{\pi\left(C_{s, 1}\right)}, \cdots, \widetilde{\pi\left(C_{s, N}\right)}\right)$ and $\widetilde{\gamma_{2 N+1}}$ is an analytic contour containing $\widehat{\{\xi(s)\}}$ inside such that $\mathbf{0} \notin \bigcup_{k=1}^{2 N+1} \widetilde{\gamma_{k}}$.

By virtue of (8.4), $h_{t, s}(\zeta)$ converges to 0 as $t \downarrow s$ uniformly in $\zeta$ on each $\widetilde{\gamma}_{k}, 1 \leq$ $k \leq 2 N+1$, and consequently we get $\lim _{t \downarrow s} a_{t}=a_{s}$ from (8.5). This together with Corollary 6.3 yields

Theorem 8.4. $a_{t}$ is a strictly increasing continuous function in $t \in\left[0, t_{\gamma}\right]$ with $a_{0}=0$.

We next denote by $\mathcal{D}$ the collection of 'labeled (ordered)' standard slit domains. For instance, $\mathbb{H} \backslash\left\{C_{1}, C_{2}, C_{3}, \cdots, C_{N}\right\}$ and $\mathbb{H} \backslash\left\{C_{2}, C_{1}, C_{3}, \cdots, C_{N}\right\}$ are considered as different elements of $\mathcal{D}$ in general although they correspond to the same subset $\mathbb{H} \backslash \bigcup_{i=1}^{N} C_{i}$ of $\mathbb{H}$. For $D, \widetilde{D} \in \mathcal{D}$, define their distance $d(D, \widetilde{D})$ by

$$
d(D, \widetilde{D})=\max _{1 \leq i \leq N}\left(\left|z_{i}-\widetilde{z}_{i}\right|+\left|z_{i}^{\prime}-\widetilde{z}_{i}^{\prime}\right|\right),
$$

where, for $D=\mathbb{H} \backslash\left\{C_{1}, C_{2}, \cdots, C_{N}\right\}, z_{i}$ (resp. $z_{i}^{\prime}$ ) denotes the left (resp. right) endpoint of $C_{i}, 1 \leq i \leq N$. $\widetilde{z}_{i}, \widetilde{z}_{i}^{\prime}, 1 \leq i \leq N$, are the corresponding points to $\widetilde{D}$. $\left\{D_{t}: 0 \leq t \leq t_{\gamma}\right\}$ is a one parameter subfamily of $\mathcal{D}$.

The following theorem follows immediately from Theorems 8.18 .2

Theorem 8.5. $\left\{D_{t}: 0 \leq t \leq t_{\gamma}\right\}$ is continuous in $t$ in the sense that, for any $\varepsilon>0$, there exists $\delta>0$ such that, for any $s$ with $|t-s|<\delta, d\left(D_{t}, D_{s}\right)<\varepsilon$.

Theorem 8.6. $\xi(t)$ is continuous in $t \in\left[0, t_{\gamma}\right]$. Moreover,

$$
\lim _{t \downarrow s} \beta_{0}(t, s)=\xi(s), \quad \lim _{t \downarrow s} \beta_{1}(t, s)=\xi(s) .
$$


Just as in Duren [8, p. 85], the left continuity of $\xi(t)$ follows from Theorem 8.1 . while its right continuity as well as (8.7) follow from Theorem 8.2. So the proof of Theorem 8.6 is omitted.

\section{LipsChitz CONTINUITY OF $\Psi$ AND DIFFERENTIABILITY OF $g_{t}$}

The goal of this section is to establish the following theorem, which combined with the theorems obtained in the preceding section will enable us to derive the Komatu-Loewner differential equation (1.4) for the map $g_{t}(z)$ but with the left derivative $\frac{\partial^{-}}{\partial t}$ being replaced by the derivative $\frac{\partial}{\partial t}$.

Recall the distance $d$ defined by (8.6) on the space $\mathcal{D}$ of all 'labeled' standard slit domains. For each $D \in \mathcal{D}$, the associated BMD-complex Poisson kernel $\Psi(z, \zeta)$, $(z, \zeta) \in D \times \partial \mathbb{H}$, is well defined as in $\S 6.1$. Recall also that, for $D=\mathbb{H} \backslash K, K=$ $\bigcup_{i=1}^{N} C_{i}, C_{i}^{+}$(resp. $C_{i}^{-}$) denotes the upper (resp. lower) side of the slit $C_{i}$, and $\partial_{p} K$ denotes the set $\bigcup_{i=1}^{N}\left(C_{i}^{+} \cup C_{i}^{-}\right)$with topology induced from the path distance on $\mathbb{H} \backslash K$. By Lemma 6.1, $\Psi(z, \zeta)$ can be extended to be a continuous function on $\left(D \cup \partial_{p} K \cup(\partial \mathbb{H} \backslash J)\right) \times J$ for any compact interval $J \subset \partial \mathbb{H}$.

Theorem 9.1. The correspondence $\mathcal{D} \mapsto \Psi(z, \zeta)$ is Lipschitz continuous in the following sense: Let $U_{j}, V_{j}, 1 \leq j \leq N$, be any relatively compact open subset of $\mathbb{H}$ with

$$
\bar{U}_{j} \subset V_{j} \subset \bar{V}_{j} \subset \mathbb{H} \quad \text { and } \quad \bar{V}_{j} \cap \bar{V}_{k}=\emptyset \text { for } j \neq k .
$$

We fix any $a>0$ and $b>0$ so that the subcollection $\mathcal{D}_{0}$ of $\mathcal{D}$ defined by

$$
\mathcal{D}_{0}=\left\{\mathbb{H} \backslash \bigcup_{j=1}^{N} C_{j} \in \mathcal{D}: C_{j} \subset U_{j},\left|z_{j}-z_{j}^{\prime}\right|>a, \operatorname{dist}\left(C_{j}, \partial U_{j}\right)>b, 1 \leq j \leq N\right\}
$$

is non-empty. There exists $\varepsilon_{0}>0$ such that for any $\varepsilon \in\left(0, \varepsilon_{0}\right)$ and for any $D \in \mathcal{D}_{0}$ and $\widetilde{D} \in \mathcal{D}$ with $d(D, \widetilde{D})<\varepsilon$, there exists a diffeomorphism $\widetilde{f}_{\varepsilon}$ from $\mathbb{H}$ onto $\mathbb{H}$ satisfying

(i) $\widetilde{f}_{\varepsilon}$ is sending $D$ onto $\widetilde{D}$, linear on $\bigcup_{j=1}^{N} U_{j}$ and the identity map on $\mathbb{H} \backslash$ $\bigcup_{j=1}^{N} \bar{V}_{j}$

(ii) for some positive constant $L_{1}$ independent of $\varepsilon \in\left(0, \varepsilon_{0}\right), D \in \mathcal{D}_{0}$ and $\widetilde{D} \in \mathcal{D}$,

$$
\left|z-\widetilde{f}_{\varepsilon}(z)\right| \leq L_{1} \varepsilon, \quad z \in \mathbb{H}
$$

(iii) for any compact subset $Q$ of $\overline{\mathbb{H}}$ containing $\bigcup_{j=1}^{N} U_{j}$ and for any compact subset $J$ of $\partial \mathbb{H}$,

$$
\left|\Psi(z, \zeta)-\widetilde{\Psi}\left(\widetilde{f}_{\varepsilon}(z), \zeta\right)\right| \leq L_{Q, J} \cdot \varepsilon, \quad z \in(Q \backslash K) \cup \partial_{p} K, \quad \zeta \in J,
$$

where $\widetilde{\Psi}$ denotes the BMD-complex Poisson kernel for $\widetilde{D}$ and $L_{Q, J}$ is a positive constant independent of $\varepsilon \in\left(0, \varepsilon_{0}\right), D \in \mathcal{D}_{0}$ and $\widetilde{D} \in \mathcal{D}$.

The proof of Theorem 9.1 will be carried out through Lemmas 9.2, 9.5 and 9.7. 
Let $U_{j}$ and $V_{j}$ be as in the statement of Theorem 9.1. For any $\varepsilon>0$ and any $D \in \mathcal{D}_{0}$, take any $\widetilde{D} \in \mathcal{D}$ with $d(D, \widetilde{D})<\varepsilon$. The quantities associated with $\widetilde{D}$ will be designated with $\sim$. For each $1 \leq j \leq N$, let $\delta_{j} \in \mathbb{R}, b_{j} \in \mathbb{C}$ be constants that are uniquely determined by

$$
\left\{\begin{array}{l}
\widetilde{z}_{j}-z_{j}=\delta_{j} z_{j}+b_{j}, \\
\widetilde{z}_{j}^{\prime}-z_{j}^{\prime}=\delta_{j} z_{j}^{\prime}+b_{j},
\end{array}\right.
$$

where $z_{j}=x_{j 1}+i x_{j 2}$ (resp. $z_{j}^{\prime}=x_{j 1}^{\prime}+i x_{j 2}^{\prime}$ ) is the left (resp. right) endpoint of the slit $C_{j}$. Since $\delta_{j}=\frac{\left(\widetilde{x}_{j 1}-x_{j 1}\right)+\left(x_{j 1}^{\prime}-\widetilde{x}_{j 1}^{\prime}\right)}{x_{j 1}-x_{j 1}^{\prime}}$ and $\left|b_{j}\right| \leq\left|\widetilde{z}_{j}-z_{j}\right|+\left|\delta_{j}\right|\left|z_{j}\right|$, we have

$$
\frac{\left|\delta_{j}\right|}{\varepsilon} \leq \frac{2}{a}, \quad \frac{\left|b_{j}\right|}{\varepsilon} \leq 1+\frac{2 M}{a}, \quad \text { where } M=\sup _{z \in \bigcup_{j=1}^{N} U_{j}}|z| .
$$

Define a linear map

$$
F_{j, \varepsilon}(z)=\frac{1}{\varepsilon}\left(\delta_{j} z+b_{j}\right), \quad 1 \leq j \leq N
$$

whose coefficients are bounded uniformly in $\varepsilon>0, D \in \mathcal{D}_{0}$ and $\widetilde{D} \in \mathcal{D}$ by (9.5). Choose a smooth function $q\left(x_{1}, x_{2}\right), z=x_{1}+i x_{2} \in \mathbb{H}$, taking value in $[0,1]$ such that

$$
q\left(x_{1}, x_{2}\right)= \begin{cases}1 & \text { if } x_{1}+i x_{2} \in U_{j}, \quad 1 \leq j \leq N, \\ 0 & \text { if } x_{1}+i x_{2} \in \mathbb{H} \backslash \bigcup_{j=1}^{N} \bar{V}_{j},\end{cases}
$$

and define a map $\widetilde{f}_{\varepsilon}$ by

$$
\left\{\begin{array}{l}
\tilde{f}_{\varepsilon}(z)=z+\varepsilon F_{\varepsilon}\left(x_{1}, x_{2}\right), \quad \text { where } \\
F_{\varepsilon}\left(x_{1}, x_{2}\right)=q\left(x_{1}, x_{2}\right) \sum_{j=1}^{N} \mathbf{1}_{V_{j}}(z) F_{j, \varepsilon}(z), \quad z=x_{1}+i x_{2} .
\end{array}\right.
$$

Lemma 9.2. There exists $\varepsilon_{0}>0$ such that, for any $\varepsilon \in\left(0, \varepsilon_{0}\right)$ and for any $D \in \mathcal{D}_{0}$ and $\widetilde{D} \in \mathcal{D}$ with $d(D, \widetilde{D})<\varepsilon$, the map $\widetilde{f}_{\varepsilon}$ defined by (9.7) is a diffeomorphism from $\mathbb{H}$ onto $\mathbb{H}$ satisfying properties (i) and (ii) of Theorem 9.1 .

Proof. In view of (9.5), (9.6) and (9.7), $F_{\varepsilon}\left(x_{1}, x_{2}\right)$ and its derivatives are bounded on $\mathbb{H}$ uniformly in $\varepsilon>0, D \in \mathcal{D}_{0}$ and $\widetilde{D} \in \mathcal{D}$. So we can find $\varepsilon_{1}>0$ independent of $D \in \mathcal{D}_{0}$ such that (9.7) defines a continuous one-to-one map $\widetilde{f}_{\varepsilon}$ from $\mathbb{H}$ into $\mathbb{H}$ for any $\varepsilon \in\left(0, \varepsilon_{1}\right) . \widetilde{f}_{\varepsilon}$ is linear on each $U_{j}$, sends $C_{j}$ onto $\widetilde{C}_{j}$ for $1 \leq j \leq N$, and an identity map on $\mathbb{H} \backslash \bigcup_{j=1}^{N} \bar{V}_{j}$. Further, it satisfies (9.3) for a constant $L_{1}$ independent of $\varepsilon>0$, $D \in \mathcal{D}_{0}$ and $\widetilde{D} \in \mathcal{D}$. In what follows, we write $\widetilde{f}_{\varepsilon}(z)=\widetilde{z}, z=x_{1}+i x_{2}, \widetilde{z}=\widetilde{x}_{1}+i \widetilde{x}_{2}$. We then have

$$
\frac{\partial\left(\widetilde{x}_{1}, \widetilde{x}_{2}\right)}{\partial\left(x_{1}, x_{2}\right)}= \begin{cases}1+\varepsilon L\left(x_{1}, x_{2}\right) & \text { if } x_{1}+i x_{2} \in \bigcup_{j=1}^{N} \bar{V}_{j}, \\ 1 & \text { if } x_{1}+i x_{2} \in \mathbb{H} \backslash \bigcup_{j=1}^{N} \bar{V}_{j},\end{cases}
$$

where $L\left(x_{1}, x_{2}\right)$ is a uniformly bounded function on $\bigcup_{j=1}^{N} \bar{V}_{j}$ in $\varepsilon>0, D \in \mathcal{D}_{0}$ and $\widetilde{D} \in \mathcal{D}$. Hence $\frac{\partial\left(\widetilde{x}_{1}, \widetilde{x}_{2}\right)}{\partial\left(x_{1}, x_{2}\right)}>0$ for $x_{1}+i x_{2} \in \mathbb{H}$, and $\widetilde{f}_{\varepsilon}$ is an open map for any $\varepsilon \in\left(0, \varepsilon_{2}\right)$ for some $\varepsilon_{2}$ independent of $D \in \mathcal{D}_{0}$. We let $\varepsilon_{0}=\varepsilon_{1} \wedge \varepsilon_{2}$. For $\varepsilon \in\left(0, \varepsilon_{0}\right)$, $\widetilde{U}=\widetilde{f}_{\varepsilon}(\mathbb{H})$ is a connected open subset of $\mathbb{H}$. On the other hand, note that $f_{\varepsilon}$ is 
an identity map on the relative closure of $\mathbb{H} \backslash \bigcup_{j=1}^{N} \bar{V}_{j}$ in $\mathbb{H}$. Since $\bigcup_{j=1}^{N} \bar{V}_{j}$ is a compact subset of $\mathbb{H}$, its image under $f_{\varepsilon}$ is also compact. It follows that

$$
\widetilde{U}=f(\mathbb{H})=\left(\overline{\mathbb{H} \cap \bar{H} \backslash \bigcup_{j=1}^{N} \bar{V}_{j}}\right) \cup f\left(\bigcup_{j=1}^{N} \bar{V}_{j}\right)
$$

is relatively closed in $\mathbb{H}$. Hence $\widetilde{U}=\mathbb{H}$.

We remark that $F_{\varepsilon}$ in (9.7) depends on $\varepsilon>0, D$ and $\widetilde{D}$, while Garabedian [10, §15.1] treated a case for a fixed map from $D$ independent of $\varepsilon>0$.

We denote by $G(z, w)$ the Green function of the domain $D$ defined by (4.8) but with the superscript ${ }^{0}$ dropped. The Green function of $\widetilde{D}$ is denoted by $\widetilde{G}(\widetilde{z}, \widetilde{w})$ and we define

$$
g(z, w, \varepsilon)=\widetilde{G}\left(\widetilde{f}_{\varepsilon}(z), \widetilde{f}_{\varepsilon}(w)\right), \quad z, w \in D .
$$

We introduce a second-order self-adjoint elliptic differential operator $A_{\varepsilon}$ by

$$
\left\{\begin{array}{l}
\left(A_{\varepsilon} u\right)\left(x_{1}, x_{2}\right)=\sum_{k, \ell=1}^{2} \frac{\partial}{\partial x_{k}}\left(A_{k \ell}^{(\varepsilon)} \frac{\partial u}{\partial x_{\ell}}\right), \quad \text { where } \\
A_{k \ell}^{(\varepsilon)}=\frac{1}{2} \frac{\partial\left(\widetilde{x}_{1}, \widetilde{x}_{2}\right)}{\partial\left(x_{1}, x_{2}\right)} \sum_{j=1}^{2} \frac{\partial x_{k}}{\partial \widetilde{x}_{j}} \frac{\partial x_{\ell}}{\partial \widetilde{x}_{j}}, \quad 1 \leq k, \ell \leq 2 .
\end{array}\right.
$$

Proposition 9.3. $\quad$ (i) $g(z, w, \varepsilon)$ is a fundamental solution of $A_{\varepsilon}$ in the sense that

$$
A_{\varepsilon}\left(g_{\varepsilon} f\right)(z)=-f(z), \quad z \in D,
$$

for any $f \in C_{c}(D)$, where $\left(g_{\varepsilon} f\right)(z)=\int_{D} g(z, w, \varepsilon) f(w) d w_{1} d w_{2}$.

(ii) $A_{\varepsilon}=\frac{1}{2} \Delta+\varepsilon B^{(\varepsilon)}$, where

$$
B^{(\varepsilon)}=\sum_{k, \ell=1}^{2} b_{k \ell}^{(\varepsilon)} \frac{\partial^{2}}{\partial x_{k} \partial x_{\ell}}+\sum_{k, \ell=1}^{2} \frac{\partial b_{k \ell}^{(\varepsilon)}}{\partial x_{k}} \frac{\partial}{\partial x_{\ell}} .
$$

Here $b_{k \ell}^{(\varepsilon)}, 1 \leq k, \ell \leq 2$, are smooth functions on $\mathbb{H}$ with $b_{k \ell}^{(\varepsilon)}=b_{\ell k}^{(\varepsilon)}$ vanishing on $\left(\mathbb{H} \backslash \bigcup_{i=1}^{2} \bar{V}_{i}\right) \cup\left(\bigcup_{i=1}^{N} U_{i}\right)$ that together with their derivatives are uniformly bounded on $\mathbb{H}$ in $\varepsilon \in\left(0, \varepsilon_{0}\right), D \in \mathcal{D}_{0}$ and $\widetilde{D} \in \mathcal{D}$.

(iii) Put $F=\bigcup_{i=1}^{N}\left(\bar{V}_{i} \backslash U_{i}\right)$. Then for any $\zeta \in \overline{\mathbb{H}} \backslash F$ and $w \in \overline{\mathbb{H}}$,

$g(\zeta, w, \varepsilon)-G(\zeta, w)=\varepsilon \int_{F} B_{z}^{(\varepsilon)} G(z, \zeta) g(z, w, \varepsilon) d x_{1} d x_{2}, \quad z=x_{1}+i x_{2}, \quad \varepsilon \in\left(0, \varepsilon_{0}\right)$.

(iv) There exists $\widetilde{\varepsilon}_{0} \in\left(0, \varepsilon_{0}\right]$ independent of $D \in \mathcal{D}_{0}$ such that for any $\zeta \in \overline{\mathbb{H}} \backslash F$ and $w \in \overline{\mathbb{H}}$,

$$
g(\zeta, w, \varepsilon)-G(\zeta, w)=\varepsilon \int_{F} B_{z}^{(\varepsilon)} G(z, \zeta)\left(G(z, w)+\varepsilon \eta^{(\varepsilon)}(z, w)\right) d x_{1} d x_{2}, \quad \varepsilon \in\left(0, \widetilde{\varepsilon}_{0}\right),
$$

where $\eta^{(\varepsilon)}$ is a continuous function on $\overline{\mathbb{H}} \times \overline{\mathbb{H}}$ that is uniformly bounded in $\varepsilon \in\left(0, \widetilde{\varepsilon}_{0}\right), D \in \mathcal{D}_{0}$ and $\widetilde{D} \in \mathcal{D}$.

(v) For each compact $J \subset \partial \mathbb{H}$, the function $\frac{\partial}{\partial \mathbf{n}_{\zeta}} B_{z}^{(\varepsilon)} G(z, \zeta)$ is bounded in $(z, \zeta) \in F \times J$ that is uniform in $\varepsilon \in\left(0, \varepsilon_{0}\right), D \in \mathcal{D}_{0}$ and $\widetilde{D} \in \mathcal{D}$. 
(vi) For each $1 \leq i \leq N, B_{z}^{(\varepsilon)} \varphi^{(i)}(z)$ is bounded in $z$ on $F$ that is uniform in $\varepsilon \in\left(0, \varepsilon_{0}\right), D \in \mathcal{D}_{0}$ and $\widetilde{D} \in \mathcal{D}$. Here $\varphi^{(i)}(z)=\mathbb{P}_{z}^{\mathbb{H}}\left(\sigma_{K}<\infty, Z_{\sigma_{K}}^{\mathbb{H}} \in C_{i}\right)$, $z \in D$, and $K=\bigcup_{j=1}^{N} C_{j}$.

(vii) For each compact set $J \subset \partial \mathbb{H}$ and for $k=1,2$,

$$
\sup _{x_{1} \in \mathbb{R}, \zeta \in J} \int_{0}^{\infty} \mathbf{1}_{F}(z)\left|\frac{\partial^{2}}{\partial x_{k} \partial \zeta_{2}} G(\zeta, z)\right| d x_{2}, \sup _{x_{2}>0, \zeta \in J} \int_{-\infty}^{\infty} \mathbf{1}_{F}(z)\left|\frac{\partial^{2}}{\partial x_{k} \partial \zeta_{2}} G(\zeta, z)\right| d x_{1}
$$

are bounded in $D \in \mathcal{D}_{0}$.

(viii) Fix $1 \leq j \leq N$. It holds for $k=1,2$ that

$$
\sup _{x_{1} \in \mathbb{R}} \int_{0}^{\infty} \mathbf{1}_{F}(z)\left|\frac{\partial}{\partial x_{k}} \varphi^{(j)}(z)\right| d x_{2}, \quad \sup _{x_{2}>0} \int_{-\infty}^{\infty} \mathbf{1}_{F}(z)\left|\frac{\partial}{\partial x_{k}} \varphi^{(j)}(z)\right| d x_{1}
$$

are bounded in $D \in \mathcal{D}_{0}$.

In what follows, the constant $\widetilde{\varepsilon}_{0}$ in the statement of (iv) above will simply be denoted as $\varepsilon_{0}$. A proof of this proposition will be given in Appendix 3 through a series of lemmas. We now proceed to prove Theorem 9.1 (iii) with $\widetilde{f}_{\varepsilon}$ defined by (9.7), using the perturbation formulae (9.12) and (9.13).

For a smooth function $u$ on $D \cup \partial \mathbb{H}$ and a smooth simple curve $\gamma \subset D \cup \partial \mathbb{H}$, define

$$
I(u ; \gamma)=\int_{\gamma} \frac{\partial u(w)}{\partial \mathbf{n}_{w}} d s(w) .
$$

The corresponding quantity for $\widetilde{D}$ is denoted by $\widetilde{I}(\widetilde{u} ; \widetilde{\gamma})$.

Lemma 9.4. For a smooth simple curve $\gamma$ in $D \cup \partial \mathbb{H}$ of finite length and a smooth function $\widetilde{u}$ on $\widetilde{D} \cup \partial \mathbb{H}$, define $\widetilde{\gamma}=\widetilde{f}_{\varepsilon}(\gamma)$. Then

$$
\widetilde{I}(\widetilde{u} ; \widetilde{\gamma})=2 \int_{\gamma} \sum_{k, \ell=1}^{2} A_{k \ell}^{(\varepsilon)}(w) \frac{\partial \widetilde{u}\left(\widetilde{f}_{\varepsilon}(w)\right)}{\partial w_{k}} \frac{\partial w_{\ell}}{\partial \mathbf{n}} d s(w) .
$$

Proof. (9.17) can be established by a direct computation via a change of variable. Here we provide a different proof using Green's first identity. Extend $\gamma$ to a smooth simple closed curve in $D \cup \partial \mathbb{H}$ and denote its enclosed interior by $U$. Define $\widetilde{U}=$ $\widetilde{f}_{\varepsilon}(U)$. By Green's first formula for the self-adjoint operator $A_{\varepsilon}$,

$$
\sum_{i, j=1}^{2} \int_{U} A_{i j}^{(\varepsilon)} \frac{\partial \widetilde{v}}{\partial x_{i}} \frac{\partial \widetilde{u}}{\partial x_{j}} d x_{1} d x_{2}+\int_{U} \widetilde{v} A_{\varepsilon} \widetilde{u} d x_{1} d x_{2}=\int_{\partial U} \widetilde{v} \sum_{i, j=1}^{2} A_{i j}^{(\varepsilon)} \frac{\partial \widetilde{u}}{\partial x_{i}} \frac{\partial x_{j}}{\partial \mathbf{n}} d s .
$$

In view of the proof of Lemma 12.1 below, the left-hand side equals

$$
\frac{1}{2} \int_{\widetilde{U}} \sum_{j=1}^{2} \frac{\partial \widetilde{v}}{\partial \widetilde{x}_{j}} \frac{\partial \widetilde{u}}{\partial \widetilde{x}_{j}} d \widetilde{x}_{1} d \widetilde{x}_{2}+\frac{1}{2} \int_{\widetilde{U}} \widetilde{v} \widetilde{\Delta} \widetilde{u} d \widetilde{x}_{1} d \widetilde{x}_{2}=\frac{1}{2} \int_{\partial \widetilde{U}} \widetilde{v} \frac{\partial \widetilde{u}}{\partial \widetilde{\mathbf{n}}} d \widetilde{s} .
$$

Therefore the right-hand sides of the above two identities coincide for any bounded smooth functions $\widetilde{v}$. A measure theoretical argument shows that they remain the same for any bounded Borel function $\widetilde{v}$ on $\partial \widetilde{U}$. Taking $\widetilde{v}$ to be the indicator function of $\widetilde{\gamma}$ establishes (9.17). 
For a function $\phi(z, \zeta)$ on $\left((\overline{\mathbb{H}} \backslash K) \cup \partial_{p} K\right) \times \partial \mathbb{H}$ and a function $\psi(z, \zeta, \varepsilon)$ on $\left((\overline{\mathbb{H}} \backslash K) \cup \partial_{p} K\right) \times \partial \mathbb{H} \times\left(0, \varepsilon_{0}\right)$, we write

$$
\phi(z, \zeta) \sim \psi(z, \zeta, \varepsilon), \quad z \in(\overline{\mathbb{H}} \backslash K) \cup \partial_{p} K, \quad \zeta \in \partial \mathbb{H},
$$

if, for any compact set $Q \subset \overline{\mathbb{H}}$ containing $\bigcup_{j=1}^{N} U_{j}$ and any compact set $J \subset \partial \mathbb{H}$, there exists a positive constant $L_{Q, J}$ independent of $\varepsilon \in\left(0, \varepsilon_{0}\right), D \in \mathcal{D}_{0}$ and $\widetilde{D}=\widetilde{f}_{\varepsilon}(D) \in \mathcal{D}$ such that

$$
|\phi(z, \zeta)-\psi(z, \zeta, \varepsilon)| \leq L_{Q, J} \cdot \varepsilon, \quad z \in(Q \backslash K) \cup \partial_{p} K, \zeta \in J, \varepsilon \in\left(0, \varepsilon_{0}\right) .
$$

Using this notation, the third assertion of Theorem 9.1 can be simply expressed as $\Psi(z, \zeta) \sim \widetilde{\Psi}\left(\widetilde{f}_{\varepsilon}(z), \zeta\right), z \in(\mathbb{H} \backslash K) \cup \partial_{p} K, \zeta \in \partial \mathbb{H}$.

We also use the obvious analogous notation $u(z) \sim v(z, \varepsilon), z \in(\overline{\mathbb{H}} \backslash K) \cup \partial_{p} K$, for functions on $(\overline{\mathbb{H}} \backslash K) \cup \partial_{p} K$, and on $(\overline{\mathbb{H}} \backslash K) \cup \partial_{p} K \times\left(0, \varepsilon_{0}\right), f(\zeta) \sim g(\zeta, \varepsilon), \zeta \in \partial \mathbb{H}$, for functions on $\partial \mathbb{H}$ and on $\partial \mathbb{H} \times\left(0, \varepsilon_{0}\right)$, and further $\alpha \sim \beta(\varepsilon)$ for a constant and a function of $\varepsilon \in\left(0, \varepsilon_{0}\right)$.

Recall from (5.2) the explicit formula for the BMD-Poisson kernel $K^{*}(z, \zeta)$ for $D:$ for $z \in D, \zeta \in \partial \mathbb{H}$,

$$
K^{*}(z, \zeta)=-\frac{1}{2} \frac{\partial}{\partial \mathbf{n}_{\zeta}} G(z, \zeta)-\Phi(z) \mathcal{A}^{-1} \frac{\partial}{\partial \mathbf{n}_{\zeta}} \Phi(\zeta)^{\operatorname{tr}},
$$

where $\Phi(z)$ is the vector with entries $\varphi^{(i)}(z), 1 \leq i \leq N$, and $\mathcal{A}$ is an $N \times N$-matrix whose $(i, j)$-component $a_{i j}$ is the period of $\varphi^{(j)}$ around the slit $C_{i}, 1 \leq i, j \leq N$.

Lemma 9.5. It holds that

$$
K^{*}(z, \zeta) \sim \widetilde{K}^{*}\left(\widetilde{f}_{\varepsilon}(z), \zeta\right), \quad z \in(\overline{\mathbb{H}} \backslash K) \cup \partial_{p} K, \quad \zeta \in \partial \mathbb{H} .
$$

Proof. By taking the partial derivative in $\zeta_{2}$ at $\zeta \in \partial \mathbb{H}$ on both sides of (9.13) and using the symmetry of $G$, Proposition $9.3(\mathrm{v})$, as well as the property $G \leq G^{\mathbb{H I}}$, we have

$$
\frac{\partial}{\partial \mathbf{n}_{\zeta}} G(w, \zeta) \sim \frac{\partial}{\partial \mathbf{n}_{\zeta}} \widetilde{G}\left(\widetilde{f}_{\varepsilon}(w), \zeta\right), \quad w \in(\overline{\mathbb{H}} \backslash K) \cup \partial_{p} K, \quad \zeta \in \partial \mathbb{H} .
$$

Let $\gamma_{i} \subset U_{i}$ be a smooth Jordan curve surrounding $C_{i}$ for each $1 \leq i \leq N$. By Theorem 4.5 (i), $\varphi^{(i)}(w)=-\frac{1}{2} I\left(G(\cdot, w) ; \gamma_{i}\right)$. On the other hand, since $A^{(\varepsilon)}=\frac{1}{2} \Delta$ on $U$ by Proposition 9.3 (9.17) applied to $\widetilde{u}(\cdot)=\widetilde{G}\left(\cdot, \widetilde{f}_{\varepsilon}(w)\right)$ reads

$$
\widetilde{\varphi}^{(i)}\left(\widetilde{f}_{\varepsilon}(w)\right)=-\frac{1}{2} \widetilde{I}\left(\widetilde{u} ; \widetilde{\gamma}_{i}\right)=-\frac{1}{2} I\left(g(\cdot, w, \varepsilon) ; \gamma_{i}\right),
$$

where $\widetilde{u}(\cdot)=\widetilde{G}\left(\cdot, \widetilde{f}_{\varepsilon}(w)\right)$. By taking the period around $C_{i}$ with respect to $\zeta$ on both sides of (9.13), we have by Proposition 9.3)(vi),

$$
\varphi^{(i)}(w) \sim \widetilde{\varphi}^{(i)}\left(\widetilde{f}_{\varepsilon}(w)\right), \quad w \in(\overline{\mathbb{H}} \backslash K) \cup \partial_{p} K, \quad 1 \leq i \leq N .
$$

In the rest of the proof, we will use (9.12) instead of (9.13). In both sides of (9.12), we take the period around $C_{i}$ with respect to $w$ and use (9.21) to obtain

$$
\varphi^{(i)}(\zeta)-\widetilde{\varphi}^{(i)}\left(\widetilde{f}_{\varepsilon}(\zeta)\right)=\varepsilon \int_{F} B_{z}^{(\varepsilon)} G(z, \zeta) \widetilde{\varphi}^{(i)}\left(\widetilde{f}_{\varepsilon}(z)\right) d x_{1} d x_{2}
$$

Keeping in mind that $0 \leq \widetilde{\varphi}^{(i)}\left(\tilde{f}_{\varepsilon}(z)\right) \leq 1$, we perform two operations in (9.23) with respect to $\zeta$. Firstly we take the partial derivative in $\zeta_{2}$ at $\zeta \in \partial \mathbb{H}$ and use 
Proposition 9.3 (v) to obtain

$$
\frac{\partial}{\partial \mathbf{n}_{\zeta}} \varphi^{(i)}(\zeta) \sim \frac{\partial}{\partial \mathbf{n}_{\zeta}} \widetilde{\varphi}^{(i)}\left(\tilde{f}_{\varepsilon}(\zeta)\right), \quad \zeta \in \partial \mathbb{H}, \quad 1 \leq i \leq N
$$

Secondly we take the period for the curve $\gamma_{j} \subset U_{j}$ surrounding $C_{j}$ and use (9.17) as well as Proposition 9.3 (vi) again to have

$$
a_{i j}-\widetilde{a}_{i j}=\varepsilon \eta_{i j}(\varepsilon), \quad 1 \leq i, j \leq N,
$$

for $\eta_{i j}(\varepsilon)$ bounded uniformly in $\left(0, \varepsilon_{0}\right), D \in \mathcal{D}_{0}$ and $\widetilde{f}_{\varepsilon}(D)$. This implies that $a_{i j}=a_{i j}(D)$ is, as a function of $D \in \mathcal{D}$, uniformly Lipschitz continuous on $\mathcal{D}_{0}$ and consequently

$$
b_{i j} \sim \widetilde{b}_{i j}, \quad 1 \leq i, j \leq N,
$$

for $(i, j)$-component $b_{i j}$ (resp. $\left.\widetilde{b}_{i j}\right)$ of $\mathcal{A}^{-1}$ (resp. $\widetilde{\mathcal{A}}^{-1}$ ). (9.19) follows from (9.18), (9.20), (9.22), (9.24) and (9.26).

We write $\Psi(z, \zeta)=u(z, \zeta)+i K^{*}(z, \zeta), z \in D, \zeta \in \partial \mathbb{H}$. Fix a compact interval $J \subset \partial \mathbb{H}$, take a point $z_{0}=x_{0}+i 0 \in \partial \mathbb{H}$ on the right of $J$ and consider the half line $\Gamma=\left\{z \in \partial \mathbb{H}: x_{0} \leq \Re z\right\} \subset \partial \mathbb{H}$. For each $z \in D$, let $\gamma(z)$ be any smooth simple curve in $D$ joining $z$ to $z_{0}$. As we saw in the proof of Theorem 6.1(ii), $u(z, \zeta), u \in D, \zeta \in J$, can be evaluated as

$$
u(z, \zeta)=I\left(K^{*}(\cdot, \zeta) ; \gamma(z)\right)+I\left(K^{*}(\cdot, \zeta) ; \Gamma\right),
$$

independent of the choice of the curve $\gamma(z)$.

We let $V=\bigcup_{j=1}^{N} V_{j}, U=\bigcup_{j=1}^{N} U_{j}$ so that $F=\bar{V} \backslash U$. We make special choices of $x_{0}$ and $\gamma(z)$ so that the straight line through $z_{0}$ is parallel to the $y$-axis and does not intersect with $\bar{V}$, while $\gamma(z)$ consists of at most two line segments $\gamma_{1}$ (starting at $\left.z_{0}\right), \gamma_{2}$ (ending at $z$ ) parallel to the $y$-axis and one line segment $\gamma_{3}$ parallel to the $x$-axis through the slit domain $D$. Define $\widetilde{\gamma}\left(\widetilde{f}_{\varepsilon}(z)\right)=\widetilde{f}_{\varepsilon}(\gamma(z))$.

Lemma 9.6. There exists a positive $\varepsilon_{0}^{\prime} \leq \varepsilon_{0}$ independent of $D \in \mathcal{D}_{0}$ such that, for any positive $\varepsilon<\varepsilon_{0}^{\prime}$ and for any relatively compact open set $Q$ with $\bar{V} \subset Q \subset \overline{\mathbb{H}}$, the inequality

$$
\left|\widetilde{I}\left(\widetilde{G}\left(\cdot, \widetilde{f}_{\varepsilon}(z)\right) ; \widetilde{\gamma}\left(\widetilde{f}_{\varepsilon}(w)\right)\right)\right| \leq C_{1}+C_{2} \log _{+} \frac{1}{\left|x_{1}-w_{1}\right|}+C_{3} \log _{+} \frac{1}{\left|x_{2}-w_{2}\right|}
$$

holds for any $z=x_{1}+i x_{2} \in F, w=w_{1}+i w_{2} \in \bar{Q}$ with $x_{i} \neq w_{i}$ for $i=1,2$, where $C_{1}, C_{2}, C_{3}$ are positive constants independent of $\varepsilon \in\left(0, \varepsilon_{0}^{\prime}\right), D \in \mathcal{D}_{0}$ and $\widetilde{f}_{\varepsilon}(D)$. Here $\log _{+} a:=0 \vee \log a$ for $a>0$.

Proof. Let $Q=\left(A_{1}, B_{1}\right) \times\left(0, B_{2}\right)$. By (9.17) and the definition (9.8) of $g(\cdot, \cdot, \varepsilon)$, we have

$$
\widetilde{I}\left(\widetilde{G}\left(\cdot, \widetilde{f}_{\varepsilon}(z)\right), \widetilde{\gamma}\left(\widetilde{f}_{\varepsilon}(w)\right)\right)=2 \int_{\gamma(w)} \sum_{k, \ell=1}^{2} A_{k \ell}^{(\varepsilon)} \frac{\partial g\left(z, w^{\prime}, \varepsilon\right)}{\partial w_{k}^{\prime}} \frac{\partial w_{\ell}^{\prime}}{\partial \mathbf{n}} d s\left(w^{\prime}\right),
$$

which equals $\widetilde{I}_{1}+\widetilde{I}_{2}+\widetilde{I}_{3}$, where

$$
\left\{\begin{array}{l}
\widetilde{I}_{j}=2 \sum_{k=1}^{2} \int_{\gamma_{j}} A_{k 1}^{(\varepsilon)}\left(w^{\prime}\right) g_{w_{k}^{\prime}}\left(z, w^{\prime}, \varepsilon\right) d w_{2}^{\prime}, \quad j=1,2, \\
\widetilde{I}_{3}=2 \sum_{k=1}^{2} \int_{\gamma_{3}} A_{k 2}^{(\varepsilon)}\left(w^{\prime}\right) g_{w_{k}^{\prime}}\left(z, w^{\prime}, \varepsilon\right) d w_{1}^{\prime} .
\end{array}\right.
$$


We show that $\left|\widetilde{I}_{2}\right|$ admits a bound as (9.28). The bound for $\left|\widetilde{I}_{1}\right|$ is the same but simpler, and that for $\left|\widetilde{I}_{3}\right|$ is quite analogous. Let $\beta>0$ be a uniform bound of $\left|A_{k \ell}^{(\varepsilon)}(w)\right|, w \in \mathbb{H}, 1 \leq k, \ell \leq 2$. Then

$$
\begin{aligned}
\left|\widetilde{I}_{2}\right| \leq 2 \beta\left(\widetilde{J}_{1}+\widetilde{J}_{2}\right), \text { for } \widetilde{J}_{1} & =\int_{\gamma_{2}}\left|\frac{\partial}{\partial w_{1}^{\prime}} g\left(z, w^{\prime}, \varepsilon\right)\right| d w_{2}^{\prime}, \\
\widetilde{J}_{2} & =\int_{\gamma_{2}} \mathbf{1}_{F}\left(w^{\prime}\right)\left|\frac{\partial}{\partial w_{2}^{\prime}} g\left(z, w^{\prime}, \varepsilon\right)\right| d w_{2}^{\prime} .
\end{aligned}
$$

Notice that the integrand for $\widetilde{J}_{2}$ vanishes for $w^{\prime} \in \mathbb{H} \backslash F$ because $A_{21}^{(\varepsilon)}=0$ there.

In view of (4.8),

$$
g\left(w^{\prime}, z, \varepsilon\right)=\frac{1}{2 \pi} \log \left|\widetilde{f}_{\varepsilon}\left(w^{\prime}\right)-\widetilde{f}_{\varepsilon}(z)\right|^{2}-\frac{1}{2 \pi} \int_{\partial \widetilde{D}} \widetilde{\Pi}\left(\widetilde{f}_{\varepsilon}(z), d \widetilde{z}\right) \log \left|\widetilde{z}-\widetilde{f}_{\varepsilon}\left(w^{\prime}\right)\right|^{2}
$$

where $\widetilde{\Pi}(\widetilde{\zeta}, \cdot)$ is the exit distribution by the Brownian motion starting at $\widetilde{\zeta}$ from $\widetilde{D}$. Denote by $F_{1}\left(x_{1}, x_{2}\right)$ and $F_{2}\left(x_{1}, x_{2}\right)$ the first and second components of $F_{\varepsilon}\left(x_{1}, x_{2}\right)$ defined by (9.7). They are Lipschitz continuous on $\mathbb{H}$ and we take $M>0$ larger than their Lipschitz constants. We also take $M^{\prime}>0$ to be a uniform bound of $\left|\frac{\partial}{\partial x_{k}} F_{i}\left(x_{1}, x_{2}\right)\right|, 1 \leq i, k \leq 2$. It is then easy to see that, for any positive $\varepsilon$ smaller than $\varepsilon_{1}=(8 M)^{-1} \wedge\left(M^{\prime}\right)^{-1}$ and for $z, w^{\prime} \in \mathbb{H}$,

$$
\frac{1}{2 \pi}\left|\frac{\partial}{\partial w_{k}^{\prime}} \log \right| \widetilde{f}_{\varepsilon}\left(w^{\prime}\right)-\left.\widetilde{f}_{\varepsilon}(z)\right|^{2} \mid \leq \frac{5}{\pi} \frac{\left|w_{1}^{\prime}-x_{1}\right|+\left|w_{2}^{\prime}-x_{2}\right|}{\left(w_{1}^{\prime}-x_{1}\right)^{2}+\left(w_{2}^{\prime}-x_{2}\right)^{2}}, k=1,2 .
$$

We take $\varepsilon_{0}^{\prime}=\varepsilon_{0} \wedge \varepsilon_{1}$. For $\varepsilon \in\left(0, \varepsilon_{0}^{\prime}\right)$, the contributions of the first term of the right-hand side of (9.30) to $\widetilde{J}_{k}, k=1,2$, are therefore less than (for $w \in Q, z \in$ $\left.F, w_{1} \neq x_{1}\right)$

$$
5+\frac{5}{2 \pi}\left|\log \left[\left(\left(w_{1}-x_{1}\right)^{2}+\left(B_{2}-x_{2}\right)^{2}\right)\left(\left(w_{1}-x_{1}\right)^{2}+x_{2}^{2}\right)\right]\right|+\frac{10}{\pi} \log _{+} \frac{1}{\left|w_{1}-x_{1}\right|} .
$$

On the other hand, we have for $\varepsilon \in\left(0, \varepsilon_{0}^{\prime}\right)$,

$$
\begin{gathered}
\frac{1}{2 \pi}\left|\frac{\partial}{\partial w_{1}^{\prime}} \log \right| \widetilde{z}-\left.\widetilde{f}_{\varepsilon}\left(w^{\prime}\right)\right|^{2} \mid \leq k_{1}\left(\widetilde{z}, w^{\prime}\right) \quad \text { for } \widetilde{z} \in \partial \widetilde{D}, w^{\prime} \in Q, \quad \text { where } \\
k_{1}\left(\widetilde{z}, w^{\prime}\right)=\frac{1}{\pi} \frac{2\left|\widetilde{x}_{1}-w_{1}^{\prime}-\varepsilon F_{1}\left(w^{\prime}\right)\right|+\mathbf{1}_{F}\left(w^{\prime}\right)\left|\widetilde{x}_{2}-w_{2}^{\prime}-\varepsilon F_{2}\left(w^{\prime}\right)\right|}{\left(\widetilde{x}_{1}-w_{1}^{\prime}-\varepsilon F_{1}\left(w^{\prime}\right)\right)^{2}+\left(\widetilde{x}_{2}-w_{2}^{\prime}-\varepsilon F_{2}\left(w^{\prime}\right)\right)^{2}} .
\end{gathered}
$$

For the constant $b>0$ of (9.2), we define

$$
b_{0}=b \wedge\left(\min _{1 \leq i \leq N} \operatorname{dist}\left(\partial V_{i}, \partial \mathbb{H}\right)\right)
$$

and set $U_{0}=U \cup\left\{z \in Q ; 0<y<b_{0}\right\}$. Denote $\varepsilon_{0}^{\prime} \wedge \frac{a}{4} \wedge \frac{b}{2 L_{1}} \wedge \frac{b_{0}}{4 \sqrt{2} M^{\prime \prime}}$ by $\varepsilon_{0}^{\prime}$ again for a uniform bound $M^{\prime \prime}$ of $\left|F_{1}\right|$ and $\left|F_{2}\right|$ on $\mathbb{H}$. Then, for $\widetilde{z} \in \partial \widetilde{D}$ and $w^{\prime} \in Q \backslash U_{0}, \operatorname{dist}\left(\widetilde{z}, w^{\prime}\right) \geq b_{0} / 2$ and the denominator of $k_{1}\left(\widetilde{z}, w^{\prime}\right)$ is greater than $b_{0}^{2} / 16$ for $\varepsilon \in\left(0, \varepsilon_{0}^{\prime}\right)$ so that $k_{1}\left(\widetilde{z}, w^{\prime}\right) \mathbf{1}_{Q \backslash U_{0}}\left(w^{\prime}\right) \leq M_{1}$ for any $\widetilde{z} \in \partial \widetilde{D}$ and $\varepsilon \in\left(0, \varepsilon_{0}^{\prime}\right)$ with constant $M_{1}$ depending only on $\ell_{0}, M^{\prime \prime}$ and $Q$. When $w^{\prime} \in U, \varepsilon F_{i}\left(w^{\prime}\right)=\delta_{i} w_{i}^{\prime}+b_{i}$, $i=1,2$. By (9.5), $1+\delta_{i}>\frac{1}{2}$ for $i=1,2$. Hence $\int_{0}^{B_{2}} k_{1}\left(\widetilde{z}, w^{\prime}\right) d w_{2}^{\prime} \leq 3+M_{1} B_{2}$ for $\widetilde{z} \in \partial \widetilde{D}$. The integration with respect to $\widetilde{\Pi}$ in $\widetilde{z}$ yields the same bound for the contribution to $\widetilde{J}_{1}$ as the second term of the right-hand side of (9.30). 
Similarly, we see that $\frac{1}{2 \pi}\left|\frac{\partial}{\partial w_{2}^{\prime}} \log \right| \widetilde{z}-\left.\widetilde{f}_{\varepsilon}\left(w^{\prime}\right)\right|^{2} \mid$ is dominated by $k_{2}\left(\widetilde{z}, w^{\prime}\right)$ for $\widetilde{z} \in \partial \widetilde{D}, w^{\prime} \in Q$, where $k_{2}\left(\widetilde{z}, w^{\prime}\right)$ is obtained from $k_{1}\left(\widetilde{z}, w^{\prime}\right)$ by interchanging the subscripts 1 and 2. Since $F \subset Q \backslash U_{0}$, it follows that $k_{2}\left(\widetilde{z}, w^{\prime}\right) \mathbf{1}_{F}\left(w^{\prime}\right)$ admits a bound $M_{2}$ like $M_{1}$ as above in $\widetilde{z} \in \partial \widetilde{D}$, and so the contribution to $\widetilde{J}_{2}$ of the second term on the right-hand side of (9.30) has a bound $M_{2} B_{2}$.

The constant $\varepsilon_{0}^{\prime}$ in Lemma 9.6 will be designated by $\varepsilon_{0}$ again.

Lemma 9.7. It holds that

$$
u(z, \zeta) \sim \widetilde{u}\left(\widetilde{f}_{\varepsilon}(z), \zeta\right), \quad z \in(\overline{\mathbb{H}} \backslash K) \cup \partial_{p} K, \quad \zeta \in \partial \mathbb{H} .
$$

Proof. (a) Let $L$ be a linear operator sending a smooth function $f(w)$ on $D$ to

$$
(L f)(w)=2 \int_{\gamma(w)} \sum_{k, \ell=1}^{2} A_{k l}^{(\varepsilon)}\left(w^{\prime}\right) \frac{\partial f\left(w^{\prime}\right)}{\partial w_{k}^{\prime}} \frac{\partial w_{\ell}^{\prime}}{\partial \mathbf{n}} d s\left(w^{\prime}\right), \quad w \in D,
$$

for the curve $\gamma(w)$ specified in the paragraph above Lemma 9.6. On both sides of (9.12), we take the normal derivative at $\zeta \in J$ and apply the linear operator $L$ in $w$. By virtue of (9.17) and (9.29), we have for $w \in D, \zeta \in J$,

$$
\begin{aligned}
& \widetilde{I}\left(\widetilde{G}_{\zeta_{2}}(\zeta, \cdot) ; \widetilde{\gamma}\left(\widetilde{f}_{\varepsilon}(w)\right)\right)-I\left(G_{\zeta_{2}}(\zeta, \cdot) ; \gamma(w)\right) \\
= & \varepsilon \int_{F} B_{z}^{(\varepsilon)} G(z, \zeta)_{\zeta_{2}} \widetilde{I}\left(\widetilde{G}\left(\widetilde{f}_{\varepsilon}(z), \cdot\right) ; \widetilde{\gamma}\left(\widetilde{f}_{\varepsilon}(w)\right)\right) d x_{1} d x_{2} \\
& +2 \varepsilon \sum_{k=1}^{2} \int_{\gamma_{1} \cup \gamma_{2}} \mathbf{1}_{F}\left(w^{\prime}\right) b_{k 1}^{(\varepsilon)}\left(w^{\prime}\right) G_{\zeta_{2} w_{k}^{\prime}}\left(\zeta, w^{\prime}\right) d w_{2}^{\prime} \\
& +2 \varepsilon \sum_{k=1}^{2} \int_{\gamma_{3}} \mathbf{1}_{F}\left(w^{\prime}\right) b_{k 2}^{(\varepsilon)}\left(w^{\prime}\right) G_{\zeta_{2} w_{k}^{\prime}}\left(\zeta, w^{\prime}\right) d w_{1}^{\prime} .
\end{aligned}
$$

This combined with Lemma 9.6 and Proposition 9.3 (ii), (v), (vii), yields

$$
I\left(G_{\zeta_{2}}(\zeta, \cdot) ; \gamma(w)\right) \sim \widetilde{I}\left(\widetilde{G}_{\zeta_{2}}(\zeta, \cdot) ; \widetilde{\gamma}\left(\widetilde{f}_{\varepsilon}(w)\right)\right), \quad w \in(\overline{\mathbb{H}} \backslash K) \cup \partial_{p} K, \zeta \in \partial \mathbb{H} .
$$

(b) As $g(z, w, \varepsilon)=\widetilde{G}\left(\widetilde{f}_{\varepsilon}(z), w\right) \leq G^{\mathbb{H}}\left(\widetilde{f}_{\varepsilon}(z), w\right)$ for $z \in \mathbb{H}$ and $w \in \mathbb{H} \backslash \bigcup_{j=1}^{N} \bar{V}_{j}$,

$$
I(g(z, \cdot, \varepsilon) ; \Gamma) \leq\left.\int_{-\infty}^{\infty} G_{w_{2}}^{\mathbb{H}}\left(\widetilde{f}_{\varepsilon}(z), w\right)\right|_{w_{2}=0} d w_{1}=2 .
$$

So we can use (9.12) and Proposition 9.3 (v) again to get

$$
I\left(G_{\zeta_{2}}(\zeta, \cdot) ; \Gamma\right) \sim I\left(g_{\zeta_{2}}(\zeta, \cdot, \varepsilon) ; \Gamma\right), \quad \zeta \in \partial \mathbb{H} .
$$

Since $\widetilde{f}_{\varepsilon}(z)=z$ near $\partial \mathbb{H}$, we have $\widetilde{I}\left(\widetilde{G}_{\zeta_{2}}(\zeta, \cdot) ; \Gamma\right)=I\left(g_{\zeta_{2}}(\zeta, \cdot, \varepsilon) ; \Gamma\right)$ for $\zeta \in \partial \mathbb{H}$ and

$$
I\left(G_{\zeta_{2}}(\zeta, \cdot) ; \Gamma\right) \sim \widetilde{I}\left(\widetilde{G}_{\zeta_{2}}(\zeta, \cdot) ; \Gamma\right), \quad \zeta \in \partial \mathbb{H}
$$

(c) In (a), we replace the operation of taking the partial derivative with respect to $\zeta_{2}$ at $\zeta \in \partial \mathbb{H}$ by the operation of taking a period around $C_{i}$ in $\zeta \in \gamma_{i} \subset U_{i}$ and use (9.21). We then utilize Proposition 9.3 (ii), (vi), (viii) as well as Lemma 9.6. We can also make the same replacement in (b) and use Proposition 9.3 (vi). We thus arrive at

$I\left(\varphi^{(i)} ; \gamma(w)\right) \sim \widetilde{I}\left(\widetilde{\varphi}^{(i)} ; \widetilde{\gamma}\left(\widetilde{f}_{\varepsilon}(w)\right), w \in D \cup \partial_{p} K \cup \partial \mathbb{H} \quad\right.$ and $\quad I\left(\varphi^{(i)} ; \Gamma\right) \sim \widetilde{I}\left(\widetilde{\varphi}^{(i)} ; \Gamma\right)$.

(9.32) follows from (9.27), (9.33), (9.34), (9.35) combined with (9.24) and (9.26). 
Lemmas 9.5 and 9.7 yield Theorem 9.1(iii).

Finally we return to the setting of $\S 6.2$ and $\S 6.3$. That is, $D=\mathbb{H} \backslash K$ is a fixed standard slit domain with $K=\bigcup_{i=1}^{N} C_{i}, \gamma$ is a Jordan arc satisfying (6.6), $g_{t}, t \in\left[0, t_{\gamma}\right]$, is a conformal map from $D \backslash \gamma[0, t]$ onto a standard slit domain $D_{t}=\mathbb{H} \backslash K_{t}$ with $K_{t}=\bigcup_{i=1}^{N} C_{t, i}$ satisfying (6.8), and $\Psi_{t}(z, \zeta)$ is the complex Poisson kernel of BMD on $D_{t} . \Psi_{t}(z, \zeta)$ is continuous in $z \in D_{t} \cup \partial_{p} K_{t} \cup(\partial \mathbb{H} \backslash\{\zeta\})$ for each $(t, \zeta) \in\left[0, t_{\gamma}\right] \times \partial \mathbb{H}$.

By combining Theorem 9.1 with Lemma 6.1 and Theorem 8.5, we are led to

Theorem 9.8. $\Psi_{t}(z, \zeta)$ is jointly continuous in $(t, z, \zeta)$.

Proof. Fix $t^{*} \in\left[0, t_{\gamma}\right]$ and a compact interval $J \subset \partial \mathbb{H}$. We shall apply Theorem 9.1 to the single fixed $D_{t^{*}}=\mathbb{H} \backslash \bigcup_{j=1}^{N} C_{t^{*}, j} \in \mathcal{D}_{0}$ by choosing $U_{j}, V_{j}$ as $C_{t^{*}, j} \subset U_{j} \Subset$ $V_{j} \Subset \mathbb{H}$, and $a$ and $b$ less than the minimum of the width of $C_{t^{*}, j}$ and the minimum of $\operatorname{dist}\left(C_{t^{*}, j}, \partial U_{j}\right)$, respectively. Take any $\varepsilon \in\left(0, \varepsilon_{0}\right)$ and any relatively compact open subset $G_{1}$ of $\overline{\mathbb{H}}$ such that, $K_{t^{*}} \subset G_{1}$, and, if we define $G_{2}=U_{\varepsilon}\left(G_{1}\right) \cap \overline{\mathbb{H}}, G_{3}=$ $U_{\varepsilon}\left(G_{2}\right) \cap \overline{\mathbb{H}}$, then $\bar{G}_{3} \cap J=\emptyset$. Here $U_{\varepsilon}\left(G_{i}\right)$ denotes the $\varepsilon$-neighborhood of $G_{i}, i=1,2$. We write $G_{i, t}=G_{i} \backslash K_{t}, t \in\left[0, t_{\gamma}\right], 1 \leq i \leq 3$. By virtue of Lemma 6.1, there exists $\delta>0$ such that

$$
\left|\Psi_{t^{*}}\left(z_{1}^{*}, \zeta_{1}\right)-\Psi_{t^{*}}\left(z_{2}^{*}, \zeta_{2}\right)\right|<\varepsilon
$$

for any $z_{1}^{*}, z_{2}^{*} \in G_{3, t^{*}} \cup \partial_{p} K_{t^{*}}$ with $\left|z_{1}^{*}-z_{2}^{*}\right|<\delta$ and any $\zeta_{1}, \zeta_{2} \in J$ with $\left|\zeta_{1}-\zeta_{2}\right|<\delta$.

Let $\varepsilon^{\prime}=\frac{\varepsilon \wedge \delta}{1+2 L_{1}}(<\varepsilon \wedge \delta)$. By virtue of Theorem 8.5, there exists $\delta_{0}>0$ such that

$$
t \in\left(t^{*}-\delta_{0}, t^{*}+\delta_{0}\right) \cap\left[0, t_{\gamma}\right] \Longrightarrow d\left(D_{t}, D_{t^{*}}\right)<\varepsilon^{\prime},
$$

which particularly implies that $K_{t} \subset G_{2}$ whenever $\left|t-t^{*}\right|<\delta_{0}$.

Now take any $t_{1}, t_{2} \in\left(t^{*}-\delta_{0}, t^{*}+\delta_{0}\right) \cap\left[0, t_{\gamma}\right]$, any $z_{1} \in G_{2, t_{1}} \cup \partial_{p} K_{t_{1}}, z_{2} \in$ $G_{2, t_{2}} \cup \partial_{p} K_{t_{2}}$ with $\left|z_{1}-z_{2}\right|<\varepsilon^{\prime}$ and any $\zeta_{1}, \zeta_{2} \in J$ with $\left|\zeta_{1}-\zeta_{2}\right|<\delta$. Denote by $f_{\varepsilon^{\prime}}^{i}$ the diffeomorphism sending $D_{t^{*}}$ onto $D_{t_{i}}$ that appears in Theorem 9.1 , $i=1,2$. There exist unique $z_{i}^{*} \in D_{t^{*}}$ such that $f_{\varepsilon^{\prime}}^{i}\left(z_{i}^{*}\right)=z_{i}, i=1,2$. By (9.3), $\left|z_{i}-z_{i}^{*}\right| \leq L_{1} \varepsilon^{\prime}<\varepsilon$ so that $z_{i}^{*} \in G_{3, t^{*}} \cup \partial_{p} K_{t^{*}}, i=1,2$. We further have $\left|z_{1}^{*}-z_{2}^{*}\right| \leq$ $\left|z_{1}-z_{1}^{*}\right|+\left|z_{2}-z_{2}^{*}\right|+\left|z_{1}-z_{2}\right|<2 L_{1} \varepsilon^{\prime}+\varepsilon^{\prime}=\varepsilon \wedge \delta$. Therefore we obtain from (9.4) and (9.36) the following desired estimate:

$$
\begin{aligned}
\left|\Psi_{t_{1}}\left(z_{1}, \zeta_{1}\right)-\Psi_{t_{2}}\left(z_{2}, \zeta_{2}\right)\right| \leq & \left|\Psi_{t_{1}}\left(z_{1}, \zeta_{1}\right)-\Psi_{t^{*}}\left(z_{1}^{*}, \zeta_{1}\right)\right|+\left|\Psi_{t_{2}}\left(z_{2}, \zeta_{2}\right)-\Psi_{t^{*}}\left(z_{2}^{*}, \zeta_{2}\right)\right| \\
& +\left|\Psi_{t^{*}}\left(z_{1}^{*}, \zeta_{1}\right)-\Psi_{t^{*}}\left(z_{2}^{*}, \zeta_{2}\right)\right| \\
< & \left(2 L^{*}+1\right) \varepsilon
\end{aligned}
$$

where $L^{*}=L_{\overline{G_{3}}, J}$.

Theorem 9.9. The curve $\gamma$ can be reparametrized in a way that $a_{t}=2 t, t \in$ $\left[0, t_{\gamma}\right]$. Under this parametrization, $g_{t}(z)$ is differentiable in $t \in\left[0, t_{\gamma}\right]$ for each $z \in\left(D \cup \partial_{p} K\right) \backslash \gamma\left[0, t_{\gamma}\right]$ and satisfies the Komatu-Loewner differential equation

$$
\frac{\partial g_{t}(z)}{\partial t}=-2 \pi \Psi_{t}\left(g_{t}(z), \xi(t)\right), \quad g_{0}(z)=z, \quad 0<t \leq t_{\gamma} .
$$

Proof. The stated reparametrization of $\gamma$ is possible by Theorem 8.4. Under this parametrization, equation (6.17) in Theorem 6.4 is converted into (1.4). We notice that, in view of the proof of Theorem 6.4 equation (6.17) remains valid for any 
$z \in\left(D \cup \partial_{p} K\right) \backslash \gamma\left[0, t_{\gamma}\right]$. For a fixed $z \in\left(D \cup \partial_{p} K\right) \backslash \gamma\left[0, t_{\gamma}\right], \Psi_{t}\left(g_{t}(z), \xi(t)\right)$ is continuous in $t \in\left[0, t_{\gamma}\right]$ by virtue of Theorem 8.3 . Theorem 8.6 and Theorem 9.8 . Therefore (1.4) means that the left derivative of $g_{t}(z)$ in $t$ is continuous on $\left(0, t_{\gamma}\right]$ possessing a limit at $t=0$, and accordingly $g_{t}(z)$ is differentiable in $t$ on $\left(0, t_{\gamma}\right)$ and right differentiable at 0 as well (see e.g. [14, Lemma 4.3]). Equation (9.38) is established.

\section{Appendix 1: Proof of Theorem 7.1}

We prove Theorem 7.1 through a series of lemmas. We consider a strip $\mathbb{H}_{r}=$ $\{z=x+i y: 0<y<r\}$ and take a large $r>0$ so that $\mathbb{H}_{r} \supset F \cup K$. We put $D_{r}=\mathbb{H}_{r} \backslash K$. Then $D_{r} \backslash F=\mathbb{H}_{r} \backslash(F \cup K)$. Recall that $\Gamma_{r}:=\{z=x+i y: y=r\}$.

Lemma 10.1. Let

$$
v_{D_{r} \backslash F}(z)=\mathbb{P}_{z}^{\mathbb{H}}\left(\sigma_{\Gamma_{r}}<\sigma_{F \cup K}\right), \quad z \in D_{r} \backslash F .
$$

Then the limit

$$
v(z)=\lim _{r \rightarrow \infty} r v_{D_{r} \backslash F}(z), \quad z \in D,
$$

exists and admits the expression (7.4).

Proof. Obviously

$$
v_{\mathbb{H}_{r}}(z)=\mathbb{P}_{z}^{\mathbb{H}}\left(Z_{\sigma_{\Gamma_{r}}}<\infty\right)=\frac{y}{r}, \quad z=x+i y \in \mathbb{H}_{r} .
$$

By the strong Markov property of $Z^{\mathbb{H}}$,

$$
v_{\mathbb{H}_{r}}(z)=v_{D_{r} \backslash F}(z)+\mathbb{E}_{z}^{\mathbb{H}}\left[v_{\mathbb{H}_{r}}\left(Z_{\sigma_{F \cup K}}^{\mathbb{H}}\right) ; \sigma_{F \cup K}<\sigma_{\Gamma_{r}}\right] .
$$

Consequently the limit (10.2) exists and satisfies (7.4).

Let $E=\mathbb{H} \backslash F$. We consider the $\operatorname{BMD} Z^{*}=\left(Z_{t}^{*}, \mathbb{P}_{z}^{*}\right)$ on $E^{*}=(E \backslash K) \cup$ $\left\{a_{1}^{*}, \cdots, a_{N}^{*}\right\}$ obtained from the $\mathrm{ABM}$ on $E$ by rendering each compact continuum $A_{j} \subset E$ into a one point $a_{j}^{*}, 1 \leq j \leq N$. As we have observed in $\S 3.1, Z^{*}$ is nothing but the part process on $E^{*}$ of the BMD $Z^{\mathbb{H}, *}$ on $D^{*}=(\mathbb{H} \backslash K) \cup\left\{a_{1}, \cdots a_{N}^{*}\right\}$. In particular, $\nu_{i}$ and $q_{i j}^{*}$ defined by (7.6) and (7.8) can be rewritten in terms of $Z^{*}$ as

$$
\begin{gathered}
\nu_{i}(d z)=\mathbb{P}_{a_{i}^{*}}^{*}\left(Z_{\sigma_{\eta_{i}}}^{*} \in d z\right), \quad 1 \leq i \leq N, \\
q_{i j}^{*}= \begin{cases}\mathbb{P}_{a_{i}^{*}}^{*}\left(\sigma_{K^{*}}<\infty, Z_{\sigma_{K^{*}}}^{*}=a_{j}^{*}\right) /\left(1-R_{i}^{*}\right) & \text { if } i \neq j, \quad \text { if } i=j, \\
0 & \text { is } i, j \leq N,\end{cases}
\end{gathered}
$$

where $R_{i}^{*}, 1 \leq i \leq N$, are defined in the same way as (7.7). Then $0<R_{i}^{*}<1$ for $1 \leq i \leq N$. Since

$$
\sum_{j: j \neq i} q_{i j}^{*}=\frac{\int_{\eta_{i}} \mathbb{P}_{z}^{*}\left(\sigma_{K^{*}}<\infty\right) \nu_{i}(d z)-R_{i}^{*}}{1-R_{i}^{*}}<1, \quad 1 \leq i \leq N,
$$

$I-Q^{*}$ admits the inverse $M$ with positive entries

$$
M_{i j}=\sum_{n=0}^{\infty} q_{i j}^{* n} \quad \text { where } \quad q_{i j}^{* n}=\left(Q^{*}\right)_{i j}^{n}, 1 \leq i, j \leq N .
$$

Lemma 10.2. The limit $v^{*}(z)$ in (7.2) exists for $z \in D^{*} \backslash F$ and satisfies (7.3) with (7.4) and (7.5). 
Proof. Denote by $\zeta^{*}$ the lifetime of the BMD $Z^{*}$ on $E^{*}$ and let

$$
v_{r}^{*}(z):=\mathbb{P}_{z}^{*}\left(\sigma_{\Gamma_{r}}<\zeta^{*}\right), \quad z \in\left(D_{r} \backslash F\right) \cup K^{*} .
$$

Then $v_{r}^{*}(z)=\mathbb{P}_{z}^{\mathbb{H}, *}\left(\sigma_{\Gamma_{r}}<\sigma_{F}\right)$ and, for $z \in\left(D_{r} \backslash F\right) \cup K^{*}$,

$$
v_{r}^{*}(z)=v_{D_{r} \backslash F}(z)+\sum_{j=1}^{N} \mathbb{P}_{z}^{*}\left(\sigma_{K^{*}}<\sigma_{\Gamma_{r}}, Z_{\sigma_{K^{*}}}^{*}=a_{j}^{*}\right) v_{r}^{*}\left(a_{j}^{*}\right) .
$$

Since $v_{r}^{*}\left(a_{i}^{*}\right)=\int_{\eta_{i}} v_{r}^{*}(z) \nu_{i}(d z)$, and

$$
\int_{\eta_{i}} \mathbb{P}_{z}^{*}\left(\sigma_{K^{*}}<\sigma_{\Gamma_{r}}, Z_{\sigma_{K^{*}}}^{*}=a_{j}^{*}\right) \nu_{i}(d z)=\Pi_{i j}^{r *}, \quad j \neq i
$$

where $\Pi_{i j}^{r *}=\mathbb{P}_{a_{i}^{*}}^{*}\left(\sigma_{K^{*}}<\sigma_{\Gamma_{r}}, Z_{\sigma^{*}}^{r, *}=a_{j}^{*}\right)$, we get by integrating both sides of (10.8) with respect to $\nu_{i}(d z)$ that

$$
v_{r}^{*}\left(a_{i}^{*}\right)=\int_{\eta_{i}} v_{D_{r} \backslash F}(z) \nu_{i}(d z)+\sum_{j \neq i} \Pi_{i j}^{r *} v_{r}^{*}\left(a_{j}^{*}\right)+R_{i}^{r *} v_{r}^{*}\left(a_{i}^{*}\right),
$$

where $R_{i}^{r *}$ is defined by (7.7) with $F \cup \Gamma_{r}$ in place of $F$. Define for $i \neq j, q_{i j}^{r *}:=$ $\Pi_{i j}^{r *} /\left(1-R_{i}^{r *}\right)$, and

$$
f_{r}^{*}\left(a_{i}^{*}\right):=\frac{1}{1-R_{i}^{r *}} \int_{\eta_{i}} v_{D_{r} \backslash F}(z) \nu_{i}(d z), \quad 1 \leq i \leq N .
$$

Clearly $q_{i j}^{r *}>0$ for $i \neq j$,

$$
\sum_{j: j \neq i} q_{i j}^{r *}=\frac{\int_{\eta_{i}} \mathbb{P}_{z}^{*}\left(\sigma_{K^{*}}<\sigma_{\Gamma_{r}}\right) \nu_{i}(d z)-R_{i}^{r *}}{1-R_{i}^{r, *}}<1, \quad 1 \leq i \leq N,
$$

and equation (10.9) can be rewritten as

$$
v_{r}^{*}\left(a_{i}^{*}\right)-\sum_{j: j \neq i} q_{i j}^{r *} v_{r}^{*}\left(a_{j}^{*}\right)=f_{r}^{*}\left(a_{i}^{*}\right), \quad 1 \leq i \leq N .
$$

In view of (10.10), equation (10.11) admits a unique solution

$$
v_{r}^{*}\left(a_{i}^{*}\right)=\sum_{j=1}^{N} M_{i j}^{r} f_{r}^{*}\left(a_{j}^{*}\right), \quad 1 \leq i \leq N .
$$

Here $M_{i j}^{r}, 1 \leq i, j \leq N$, are the entries of the inverse of $I-Q^{r *}$ for the matrix $Q^{r *}$ with off-diagonal elements $q_{i j}^{r *}, i \neq j$, and zero diagonal elements.

Observe that, as $r \uparrow \infty, R_{i}^{r *}$ for $1 \leq i \leq N$ and $\Pi_{i j}^{r *}$ for $1 \leq i, j \leq N$ increase to $R_{i}^{*}$ and $\Pi_{i j}^{*}$, respectively. Accordingly $q_{i j}^{r *}$ and $M_{i j}^{r *}$ increase to $q_{i j}^{*}$ and $M_{i j}^{*}$, respectively. Furthermore by Lemma 10.1 .

$$
\lim _{r \rightarrow \infty} r f_{r}^{*}\left(a_{i}^{*}\right)=\frac{1}{1-R_{i}^{*}} \int_{\eta_{i}} v(z) \nu_{i}(d z), \quad 1 \leq i \leq N .
$$

Therefore we deduce from (10.12) that the limit $v^{*}\left(a_{i}^{*}\right):=\lim _{r \rightarrow \infty} r v_{r}^{*}\left(a_{i}^{*}\right)$ exists and satisfies (7.5). Moreover it follows from Lemma 10.1 and (10.8) that the limit $v^{*}(z)=\lim _{r \rightarrow \infty} r v_{r}^{*}(z)$ exists for every $z \in D$ and satisfies (17.3).

The next lemma concerns the behaviors of $v^{*}(z)$ when $|z|$ gets large. 
Lemma 10.3.

$$
\begin{aligned}
& v^{*}(x+i y)=y+o(1), \quad y \rightarrow \infty, \quad \text { uniformly in } x \in \mathbb{R} . \\
& \lim _{y \rightarrow \infty} v_{x}^{*}(x+i y)=0, \quad \text { uniformly in } x \in \mathbb{R} . \\
& \lim _{x \rightarrow \pm \infty}\left|v^{*}(x+i y)-y\right|=0, \quad \text { uniformly in } y>0 . \\
& \lim _{x \rightarrow \pm \infty}\left|v_{y}^{*}(x+i y)-1\right|=0, \quad \text { uniformly in } y>0 .
\end{aligned}
$$

Proof. (10.13) follows immediately from (17.3) and (7.4) in view of (2.12) in 13 .

Take $r_{0}>0$ such that $\mathbb{H}_{r_{0}} \supset F \cup K$. Let $p(\xi, \eta)=\frac{1}{\pi} \frac{\eta}{\xi^{2}+\eta^{2}}, \eta>0$, be the Poisson kernel of the upper half-plane for Brownian motion. In view of (17.3) and (7.4), $v^{*}(z)-y$ is a bounded harmonic function in $\left\{z=x+i y: y>r_{0}\right\}$, and so $v^{*}(x+i y)=y+\int_{-\infty}^{\infty} p\left(x-\xi, y-r_{0}\right)\left(v^{*}\left(\xi+i r_{0}\right)-r_{0}\right) d \xi, y>r_{0}$. We then get (10.14) from

$$
\left|p_{x}\left(x-\xi, y-r_{0}\right)\right| \leq \frac{2}{\left(y-r_{0}\right)} p\left(x-\xi, y-r_{0}\right) \quad \text { for } \quad y>r_{0} .
$$

Choose $\ell>0$ such that $F \cup K \subset\{z=x+i y \in \mathbb{H}:|x|<\ell\}$. Let

$$
\Lambda_{\ell}=\{z=x+i y \in \mathbb{H}: x=\ell\} .
$$

Note that it follows from (7.3) and (7.4) that $h(z):=v^{*}(z)-y$ is a bounded harmonic function on $\{z=x+i y \in \mathbb{H}:|x|>\ell\}$ vanishing continuously on the $x$-axis and admitting the expression

$$
h(z)=\mathbb{E}_{z}\left[h\left(Z_{\sigma_{\Lambda_{\ell}}}\right) ; \sigma_{\Lambda_{\ell}}<\sigma_{\partial \mathbb{H}}\right], \quad|x|>\ell .
$$

Combining this with (4.12) and (10.13), we get (10.15).

(10.16) follows from another expression of $h$ given by (4.15) for $\ell$ in place of $\ell_{1}$.

The function $v^{*}$ on $D^{*}$ constructed in Lemma 7.3 is obviously $Z^{*}$-harmonic. In view of Corollary 3.6. $-\left.v^{*}\right|_{D}$ admits a harmonic conjugate $u^{*}$ on $D$ uniquely up to an additive constant so that $f(z)=u^{*}(z)+i v^{*}(z), z \in D$, is an analytic function.

Further $u^{*}$ admits the expression as (6.4) in terms of $v^{*}$ up to a real additive constant independently of a choice of a rectifiable curve $C$ connecting $z_{0}$ with $z$. In particular, choosing $C=C_{1}+C_{2}$ for a straight line segment $C_{1}$ (resp. $C_{2}$ ) connecting $z_{0}=x_{0}+i y_{0}\left(\right.$ resp. $\left.x+i y_{0}\right)$ with $z=x+i y_{0}$ (resp. $\left.z=x+i y\right)$, we get

$$
u^{*}(x+i y)-u^{*}\left(x_{0}+i y_{0}\right)=\int_{x_{0}}^{x} v_{y}^{*}\left(\xi+i y_{0}\right) d \xi-\int_{y_{0}}^{y} v_{x}^{*}(x+i \eta) d \eta, \quad y>0 .
$$

The next lemma concerns behaviors of $f(z)$ as $|z|$ gets large.

Lemma 10.4. It holds that for each $x \in \mathbb{R}$,

$$
\lim _{y \rightarrow \infty} \frac{f(x+i y)}{x+i y}=1 .
$$

Furthermore

$$
\limsup _{z \rightarrow \infty, z \in D}\left|\frac{f(z)}{z}\right| \leq 2 .
$$


Proof. The real part of $f(x+i y) /(x+i y)$ equals $\left[x u^{*}(x+i y)+y v^{*}(x+i y)\right] /\left(x^{2}+y^{2}\right)$. As $y \rightarrow \infty$, the first term of this sum goes to zero in view of (10.14) and (10.17), while the second term goes to 1 in view of (10.13). The imaginary part equals $\left[-y u^{*}(x+i y)+x v^{*}(x+i y)\right] /\left(x^{2}+y^{2}\right)$, which goes to zero as $y \rightarrow \infty$ by the analogous observation. By (10.13) and (10.15), $\limsup _{y \rightarrow \infty} \sup _{x \in \mathbb{R}} \frac{v^{*}(z)}{|z|} \leq 1$ and $\limsup _{x \rightarrow \pm \infty} \sup _{y>0} \frac{v^{*}(z)}{|z|} \leq$ 1.

On the other hand, (10.14) and (10.16) imply that, for any $\varepsilon>0$, there exist $y_{0}>0, \ell>0$ such that

$$
\begin{gathered}
\left|v_{x}^{*}(x+i \eta)\right|<\varepsilon \quad \text { for any } x \in \mathbb{R} \text { and for any } \eta>y_{0}, \\
\left|v_{y}^{*}\left(\xi+i y_{0}\right)\right|<1+\varepsilon \quad \text { for any } \xi \text { with }|\xi|>\ell .
\end{gathered}
$$

Let $M=\sup _{|\xi| \leq \ell}\left|v_{y}^{*}\left(\xi+i y_{0}\right)\right|$. We get from (10.17) with $x_{0}=0$ that

$$
\frac{\left|u^{*}(z)\right|}{|z|} \leq \frac{\left|u^{*}\left(z_{0}\right)\right|}{|z|}+\frac{M \ell+|x|(1+\varepsilon)}{|z|}+\frac{\left|y-y_{0}\right|}{|z|} \varepsilon,
$$

and so $\limsup _{y \rightarrow \infty} \sup _{x \in \mathbb{R}} \frac{\left|u^{*}(z)\right|}{|z|} \leq 1+2 \varepsilon$. Similarly we get $\limsup _{x \rightarrow \pm \infty} \sup _{y>0} \frac{\left|u^{*}(z)\right|}{|z|} \leq 1$.

Lemma 10.5. $u^{*}$ can be chosen uniquely in such a way that $f=u^{*}+i v^{*}$ satisfies (7.9).

Proof. Since $v^{*}=0$ on $\partial(\mathbb{H} \backslash \bar{F})$, by the reflection principle, $f$ extends to be an analytic function on $\mathbb{C} \backslash((K \cup \bar{F}) \cup \pi(K \cup \bar{F}))$, which will still be denoted as $f$. Here $\pi(K \cup \bar{F})$ is the mirror reflection of $K \cup \bar{F}$ with respect to the $x$-axis.

Let $g(z):=z f(1 / z)$. Then $g$ is analytic on $\mathbb{C} \backslash\{\mathbf{0}\}$ and bounded near $\mathbf{0}$ by (10.19). So 0 is a removable singularity of $g(z)$ and $\lim _{z \rightarrow \mathbf{0}} g(z)=a_{0}$ exists. By (10.18) with $x=0, \lim _{y \downarrow 0} g(-i y)=1$ so that $a_{0}=1$ and $g$ can be expanded near zero as $g(z)=1+a_{1} z+a_{2} z^{2}+\cdots$. Therefore

$$
f(z)=z+a_{1}+\frac{a_{2}}{z}+\cdots \quad \text { near } \infty .
$$

Since $\Im f=0$ on $\partial(\mathbb{H} \backslash \bar{F}), a_{1}, a_{2}, \cdots$, are real. $f$ satisfies $(7.9)$ if and only if $\lim _{z \rightarrow \infty}(f(z)-z)=0$. The functions $f$ and $u^{*}$ are uniquely determined by $v^{*}$ under this condition.

The proof of Theorem 7.1 is now complete.

\section{Appendix 2: Proper maps And the Degree}

Recall that a continuous map $f: X \rightarrow Y$ between topological spaces is called proper if pre-images of compact sets are compact. Intuitively, if $X$ and $Y$ are subsets of larger spaces, this means that the boundary of $X$ maps into the boundary of $Y$ (though it is not required that $f$ extends continuously to the boundary). In rather general situations (for instance smooth orientation preserving maps between manifolds with boundary; see e.g. [15]) such maps have the property that every $y \in$ $Y$ has the same number of pre-images $x \in X$ (counted according to multiplicity). In this section, we formulate and prove a simple version of this principle in the setting of analytic functions that is suitable for our purpose. This is certainly standard, but lacking a reference we provide the details for the sake of completeness. We allow $\infty$ to be in the domain and in the range of $f$ and adopt the usual definition 
that a function $f$ (defined in a neighborhood of $\infty$ with $f(\infty)=\infty$ ) is analytic if $1 / f(1 / z)$ is analytic in a neighborhood of 0 .

Lemma 11.1. Let $D_{1}, D_{2}$ be connected open subsets of the Riemann sphere $\overline{\mathbb{C}}$ and $f$ analytic in $D_{1}$. If $f$ is a proper map between $D_{1}$ and $D_{2}$ (that is, if $f\left(D_{1}\right) \subset D_{2}$ and if $f^{-1}(K)$ is a compact subset of $D_{1}$ whenever $K$ is a compact subset of $\left.D_{2}\right)$, then there is a finite number $d$ such that every $w \in D_{2}$ has precisely $d$ pre-images in $D_{1}$, counting multiplicity.

Proof. Fix $w_{0}, w_{1} \in D_{2}$. In order to show that they have the same number of preimages under $f$, we first assume that neither $w_{0}$ nor $w_{1}$ are critical values (that is, we assume $f^{\prime} \neq 0$ for every pre-image of $w_{0}$ and $\left.w_{1}\right)$. Then there is a simple curve $\gamma \subset D_{2}$ joining $w_{0}$ and $w_{1}$ that is disjoint from the set of critical values. For every pre-image $z_{0}$ of $w_{0}$, the branch $g_{0}$ of the inverse function $f^{-1}$ with $g_{0}\left(w_{0}\right)=z_{0}$, defined in a neighborhood of $w_{0}$, can be analytically continued along $\gamma$ and yields a branch $g_{1}$ of $f^{-1}$ near $w_{1}$.

This is where both the assumption on the critical values and the assumption on properness are used: During the process of analytic continuation, the curve $f^{-1}(\gamma(t))$ cannot escape from $D_{1}$ by properness, and one can always analytically continue further because one does not meet critical values. Formally, one considers the subset $S=\left\{s \in[0,1]: g_{0}\right.$ can be analytically continued along $\left.\gamma[0, s]\right\}$ of the interval $[0,1]$ and shows that it is both open and closed.

Conversely, continuing $g_{1}$ along the reversed curve $\gamma^{-1}$ leads us back to $g_{0}$. Thus we have a bijection between the sets $f^{-1}\left(w_{0}\right)$ and $f^{-1}\left(w_{1}\right)$. If a pre-image $z_{0}$ (or $z_{1}$ ) of $w_{0}$ (or $w_{1}$ ) is a critical point (so that the local degree of $f$ at $z_{0}$ is more than 1 ), simply replace $w_{0}$ (or $w_{1}$ ) by nearby points $w_{0}^{\prime}$ or $w_{1}^{\prime}$ and use the fact that the number of pre-images of $w_{0}^{\prime}$ near $z_{0}$ equals the local degree of $f$ at $z_{0}$.

Now we will show that the assumption $f\left(D_{1}\right) \subset D_{2}$ can be removed if the degree is one and if the complement of $D_{2}$ has empty interior. For a function $f$ and an open set $D \subset \overline{\mathbb{C}}$, we define by

$$
f(\partial D)=\bigcap_{K \Subset D} \overline{f(D \backslash K)}
$$

the set of limit points of $f$ as $z$ approaches $\partial D$ (the intersection is over all compact subsets of $D$ ). It is easy to see that any proper map $f$ from $D_{1}$ onto $D_{2}$ satisfies $f\left(\partial D_{1}\right)=\partial D_{2}$. The next theorem goes in the opposite direction. Notice that we do not assume a priori that $f\left(D_{1}\right) \subset D_{2}$.

Theorem 11.2. Let $D_{1}, D_{2}$ be connected open subsets of the Riemann sphere $\overline{\mathbb{C}}$ and $f$ analytic in $D_{1}$. Assume that the complement of $D_{2}$ has empty interior, that

$$
f\left(\partial D_{1}\right)=\partial D_{2}
$$

and that there is one point $w_{0} \in D_{2}$ that has precisely one pre-image $z_{0}$ under $f$ (counting multiplicity). Then $f$ is a conformal map from $D_{1}$ onto $D_{2}$.

Proof. Let $D \subset D_{1}$ be a connected component of $f^{-1}\left(D_{2}\right)$. Then $f: D \rightarrow D_{2}$ is proper.

To see this, suppose $K$ is a compact subset of $D_{2}$ and $z_{n} \in f^{-1}(K) \cap D$. We need to show that every subsequential limit of $z_{n}$ is in $D$. Assume to the contrary that $z_{n}$ converges to a point $z \in \partial D$. Then if $z \in D_{1}$, it follows that $f(z) \in D_{2}$, and thus a neighborhood of $z$ belongs to $D$, a contradiction. If $z \in \partial D_{1}$, then every 
limit point of $\left\{f\left(z_{n}\right)\right\}$ belongs to $f\left(\partial D_{1}\right)=\partial D_{2}$, which contradicts the assumption that $K$ is a compact subset of $D_{2}$.

Now Lemma 11.1 implies that every $w \in D_{2}$ has the same number of pre-images in $D$. This number is trivially at least one, and it is at most one by assumption on $w_{0}$. Thus the degree of $f$ on $D$ equals one, and there is only one such component $D$. In particular, $f$ is a conformal bijection between $D$ and $D_{2}$. It remains to show that $D=D_{1}$. If not, there is a point $z_{1} \in D_{1} \cap \partial D$, and it follows that $w_{1}=f\left(z_{1}\right) \in \partial D_{2}$. By the assumption $f\left(\partial D_{1}\right)=\partial D_{2}$, there is a sequence $z_{n} \in D_{1}$ converging to a boundary point such that $f\left(z_{n}\right) \rightarrow w_{1}$. Since $f$ is an open mapping and since the complement of $D_{2}$ has empty interior, there are $z_{n}^{\prime} \in D_{1}$ so that $f\left(z_{n}^{\prime}\right) \rightarrow w_{1}$ and that $f\left(z_{n}^{\prime}\right) \in D_{2}$; in particular, $z_{n}^{\prime} \in D$. Since the equation $f(z)=f\left(z_{n}^{\prime}\right)$ has another solution near $z_{1}$, we obtain a contradiction to the injectivity of $f$ on $D$.

Notice that the last claim $D=D_{1}$ is not true if the degree is more than one, as for instance the map $f(z)=z^{2}$ with $D_{1}=D_{2}=\mathbb{C} \backslash[0,1]$ shows: Here $D=\mathbb{C} \backslash[-1,1]$.

\section{Appendix 3: Proof of Proposition 9.3}

The assertions (i) and (ii) of Proposition 9.3 will be proved by the next lemma.

Lemma 12.1. $g(z, w, \varepsilon)$ is a fundamental solution of $A_{\varepsilon}$ in the sense of (9.10). The coefficients $A_{k \ell}^{(\varepsilon)}$ of $A_{\varepsilon}$ admit expressions

$$
A_{k \ell}^{(\varepsilon)}=\frac{1}{2} \delta_{k \ell}+\varepsilon b_{k \ell}^{(\varepsilon)}, \quad 1 \leq k, \ell \leq 2,
$$

where $b_{k \ell}^{(\varepsilon)}, 1 \leq k, \ell \leq 2$, are smooth functions on $\mathbb{H}$ with $b_{k \ell}^{(\varepsilon)}=b_{\ell k}^{(\varepsilon)}$ vanishing on $\left(\mathbb{H} \backslash \bigcup_{i=1}^{N} \bar{V}_{i}\right) \cup\left(\bigcup_{i=1}^{N} U_{i}\right)$ which together with their derivatives are uniformly bounded in $\varepsilon \in\left(0, \varepsilon_{0}\right), D \in \mathcal{D}_{0}$ and $\widetilde{D}=\widetilde{f}_{\varepsilon}(D) \in \mathcal{D}$.

Proof. Denote the Jacobian determinant $\frac{\partial\left(\widetilde{x}_{1}, \widetilde{x}_{2}\right)}{\partial\left(x_{1}, x_{2}\right)}$ of the map $\widetilde{f}_{\varepsilon}$ by $J=J\left(x_{1}, x_{2}\right)$. Let $\widetilde{\mathcal{E}}$ and $\widetilde{A}$ be the Dirichlet form and the $L^{2}$-generator on $L^{2}(\widetilde{D})$ of the ABM on $\widetilde{D}$, respectively. Then $\widetilde{u} \in \mathcal{D}(\widetilde{A})$ and $\widetilde{A} \widetilde{u}=\widetilde{f} \in L^{2}(\widetilde{D})$ if and only if $\widetilde{u} \in H_{0}^{1}(\widetilde{D})$ and $\widetilde{\mathcal{E}}(\widetilde{u}, \widetilde{v})=-\frac{1}{2} \int_{\widetilde{D}} \widetilde{f} \widetilde{v} d \widetilde{x}_{1} d \widetilde{x}_{2}$. It follows from

$$
\begin{aligned}
\widetilde{\mathcal{E}}(\widetilde{u}, \widetilde{v}) & =\frac{1}{2} \int_{\widetilde{D}} \sum_{j=1}^{2} \frac{\partial \widetilde{v}}{\partial \widetilde{x}_{j}} \frac{\partial \widetilde{u}}{\partial \widetilde{x}_{j}} d \widetilde{x}_{1} d \widetilde{x}_{2} \\
& =\frac{1}{2} \int_{D} \sum_{j=1}^{2} \sum_{k, \ell=1}^{2} \frac{\partial \widetilde{v}}{\partial x_{k}} \frac{\partial x_{k}}{\partial \widetilde{x}_{j}} \frac{\partial \widetilde{u}}{\partial x_{\ell}} \frac{\partial x_{\ell}}{\partial \widetilde{x}_{j}} J\left(x_{1}, x_{2}\right) d x_{1} d x_{2} \\
& =-\int_{D} \widetilde{v}\left(x_{1}, x_{2}\right) A_{\varepsilon} \widetilde{u}\left(x_{1}, x_{2}\right) d x_{1} d x_{2}
\end{aligned}
$$

and $\int_{\widetilde{D}} \widetilde{f} \widetilde{v} d \widetilde{x}_{1} d \widetilde{x}_{2}=\int_{D} \widetilde{f}\left(x_{1}, x_{2}\right) \widetilde{v}\left(x_{1}, x_{2}\right) J\left(x_{1}, x_{2}\right) d x_{1} d x_{2}$ for every $\widetilde{v} \in C_{c}^{1}(\widetilde{D})$ that

$$
(\widetilde{A} \widetilde{u})\left(x_{1}, x_{2}\right)=J^{-1} A_{\varepsilon} \widetilde{u}\left(x_{1}, x_{2}\right) .
$$

On the other hand, if we define $\widetilde{G} \widetilde{f}(\widetilde{z})=\int_{\widetilde{D}} \widetilde{G}(\widetilde{z}, \widetilde{w}) \widetilde{f}(\widetilde{w}) d \widetilde{w}$, then

$$
\widetilde{G} \widetilde{f}(\widetilde{z})=\int_{D} g(z, w, \varepsilon)(\widetilde{f} J)(w) d w_{1} d w_{2}=: g_{\varepsilon}(\widetilde{f} J)(z) .
$$


Since $\widetilde{A}(\widetilde{G} \widetilde{f})(\widetilde{z})=-\widetilde{f}(\widetilde{z})$, we have by (12.3) that $J^{-1} A_{\varepsilon} \cdot g_{\varepsilon}(\widetilde{f} J)(z)=-\tilde{f}(z)$. This establishes (9.10) by taking $f=\widetilde{f} J$.

The stated expression and properties of coefficients of $A_{\varepsilon}$ follow from (9.7), (9.9) and the uniform boundedness of the coefficients of the linear map (9.6). In particular, $b_{k \ell}^{(\varepsilon)}, 1 \leq k, \ell \leq N$, vanish on $\mathbb{H} \backslash \bigcup_{i=1}^{N} \bar{V}_{i}$ because $\widetilde{f}_{\varepsilon}$ is an identity map there, and on each $U_{i}$ as well, because it is an analytic (actually linear) map there.

To derive the perturbation formulae (9.12) and (9.13), we first construct an appropriate parametrix for the elliptic differential operator $A_{\varepsilon}$ by following an interior variation method presented in section 15.1 of Garabedian's book 10 .

Denote by $a^{(\varepsilon)}=\left(a_{k \ell}^{(\varepsilon)}\right)_{1 \leq k, \ell \leq 2}$ the inverse matrix of $A^{(\varepsilon)}=\left(A_{k \ell}^{(\varepsilon)}\right)_{1 \leq k, \ell \leq 2}$. Since $\operatorname{det} A^{(\varepsilon)}=\frac{1}{4}$, we have

$$
a_{11}^{(\varepsilon)}=2+4 \varepsilon b_{22}^{(\varepsilon)}, \quad a_{22}^{(\varepsilon)}=2+4 \varepsilon b_{11}^{(\varepsilon)}, \quad a_{12}^{(\varepsilon)}=a_{21}^{(\varepsilon)}=-4 \varepsilon b_{12}^{(\varepsilon)} .
$$

Define

$$
\Gamma(z, \zeta)=\frac{1}{2} \sum_{i, j=1}^{2} a_{i j}^{(\varepsilon)}(\zeta)\left(x_{i}-\zeta_{i}\right)\left(x_{l}-\zeta_{j}\right), \quad z=x_{1}+i x_{2}, \quad \zeta=\zeta_{1}+i \zeta_{2} .
$$

$-\frac{1}{2 \pi} \log \Gamma(z, \zeta)$ has the same singularity as the fundamental solution $g(z, \zeta, \varepsilon)$ of the elliptic differential operator $A_{\varepsilon}$ (cf. [10, (5.80)]).

Recall the constant $b_{0}$ defined in (9.31). We fix an arbitrary $\ell_{0} \in\left(0, b_{0}\right]$ and consider a smooth non-positive real function $\alpha(t), t \in \mathbb{R}$, with

$$
\alpha(0)=-1 /(2 \pi), \quad \alpha(t)=0 \quad \text { if } \quad t \notin\left(-\ell_{0}^{2}, \ell_{0}^{2}\right) .
$$

Let

$$
\left\{\begin{array}{l}
P_{\varepsilon}(z, \zeta)=\alpha\left(|z-\zeta|^{2}\right) \log \Gamma(z, \zeta) \\
P_{0}(z, \zeta)=\alpha\left(|z-\zeta|^{2}\right) \log |z-\zeta|^{2}, \quad z, \zeta \in \overline{\mathbb{H}}, z \neq \zeta
\end{array}\right.
$$

For a function $u(z, \zeta, \varepsilon), z, \zeta \in D \cup \partial \mathbb{H}, z \neq \zeta, \varepsilon \in\left(0, \varepsilon_{0}\right)$, we write $u(z, \zeta, \varepsilon)=$ $O(\varepsilon / r), r=|z-\zeta|$, if

$$
|u(z, \zeta, \varepsilon)| \leq \varepsilon \frac{M_{1}}{|z-\zeta|}+\varepsilon M_{2}, \quad z, \zeta \in D \cup \partial \mathbb{H},
$$

for positive constants $M_{1}, M_{2}$ independent of $\varepsilon \in\left(0, \varepsilon_{0}\right), D \in \mathcal{D}_{0}$ and $\widetilde{D}=\widetilde{f}_{\varepsilon}(D) \in$ $\mathcal{D}$.

In what follows, the set $\bigcup_{i=1}^{N}\left(\bar{V}_{i} \backslash U_{i}\right)$ will be denoted by $F$.

Lemma 12.2. $G(z, \zeta)-P_{0}(z, \zeta)+P_{\varepsilon}(z, \zeta)$ is a parametrix of the operator $A_{\varepsilon}=A_{\varepsilon, z}$ in a specific sense that

$$
A_{\varepsilon, z}\left(G(z, \zeta)-P_{0}(z, \zeta)+P_{\varepsilon}(z, \zeta)\right)=O(\varepsilon / r), \quad r=|z-\zeta| .
$$

Proof. It suffices to show that

$$
\begin{aligned}
& A_{\varepsilon} P_{\varepsilon}(z, \zeta)-\frac{1}{2} \Delta P_{0}=O(\varepsilon / r), \quad r=|z-\zeta|, \\
& \left(A_{\varepsilon}-\frac{1}{2} \Delta\right)\left(G-P_{0}\right)=O(\varepsilon / r), \quad r=|z-\zeta| .
\end{aligned}
$$


Note that they imply (12.9) because $\Delta_{z} G(z, \zeta)=0$ for $z \neq \zeta$. Designating $A_{i j}^{(\varepsilon)}$ by $A_{i j}$, the left-hand side of (12.10) can be rewritten as $I_{\varepsilon}+I I_{\varepsilon}+I I I_{\varepsilon}+I V_{\varepsilon}+V_{\varepsilon}$ with

$$
\left\{\begin{array}{l}
I_{\varepsilon}=\alpha\left(|z-\zeta|^{2}\right) \sum_{i j=1}^{2} A_{i j}(z)(\log \Gamma(z, \zeta))_{x_{i} x_{j}}, \\
I I_{\varepsilon}=\sum_{i, j=1}^{2} A_{i j, x_{i}}(z)\left[\alpha\left(|z-\zeta|^{2}\right) \log \Gamma(z, \zeta)\right]_{x_{j}}, \\
I I I_{\varepsilon}=\sum_{i=1}^{2} \alpha\left(|z-\zeta|^{2}\right)_{x_{i} x_{i}}\left[A_{i i}(z) \log \Gamma(z, \zeta)-\frac{1}{2} \log |z-\zeta|^{2}\right], \\
I V_{\varepsilon}=4 \alpha^{\prime}\left(|z-\zeta|^{2}\right)\left[\sum_{i, j=1}^{2} A_{i j}(z)\left(x_{i}-\zeta_{i}\right)(\log \Gamma(z, \zeta))_{x_{j}}-1\right], \\
V_{\varepsilon}=\sum_{i \neq j} A_{i j}(z) \alpha\left(|z-\zeta|^{2}\right)_{x_{i} x_{j}} \log \Gamma(z, \zeta),
\end{array}\right.
$$

where the function $\Gamma(z, \zeta)$ is defined by (12.5). The sum $\sum_{i j=1}^{2} A_{i j}(z)(\log \Gamma(z, \zeta))_{x_{i} x_{j}}$ equals

$$
\frac{\sum_{i, j=1}^{2} A_{i j}(z) a_{i j}(\zeta) \Gamma(z, \zeta)-\sum_{i, j=1}^{2} A_{i j}(z) \Gamma_{x_{i}}(z, \zeta) \Gamma_{x_{j}}(z, \zeta)}{\Gamma(z, \zeta)^{2}}
$$

Since

$$
A_{i j}(z)=A_{i j}(\zeta)+\varepsilon \sum_{k=1}^{2} C_{i, j, k}(z, \zeta)\left(x_{k}-\zeta_{k}\right),
$$

for $C_{i, j, k}$ involving only derivatives of $b_{i j}$, and

$$
\Gamma(z, \zeta)=|z-\zeta|^{2}\left(1+\varepsilon \eta_{1}(z, \zeta)\right),
$$

for $\eta_{1}$ uniformly bounded in $\varepsilon>0, D \in \mathcal{D}_{0}$ and $\tilde{f}_{\varepsilon}(D)$ due to (12.4), we see that (12.12) can be written as

$$
\varepsilon \frac{\eta_{2}(z, \zeta)|z-\zeta| \Gamma(z, \zeta)-\eta_{3}(z, \zeta)|z-\zeta|^{3}}{\Gamma(z, \zeta)^{2}}=\varepsilon \eta_{4}(z, \zeta) \frac{1}{|z-\zeta|}
$$

for $\eta_{k}, 2 \leq k \leq 4$, uniformly bounded in $\varepsilon>0, D \in \mathcal{D}_{0}$ and $\tilde{f}_{\varepsilon}(D)$ yielding that $I_{\varepsilon}=O(\varepsilon / r)$.

All other terms $I I_{\varepsilon}, I I I_{\varepsilon}, I V_{\varepsilon}$ and $V_{\varepsilon}$ can also be verified to satisfy (12.8) due to (12.13), (12.14), Lemma 12.1 and (12.4), yielding (12.10).

By Lemma 12.1. $A_{\varepsilon}-\frac{1}{2} \Delta$ is equal to $\varepsilon B^{(\varepsilon)}$ for the differential operator $B^{(\varepsilon)}$ defined by (9.11). Using (4.7) and (12.24) below, the left-hand side of (12.11) can be written as $\varepsilon B_{z}^{(\varepsilon)} u(z, \zeta)-\varepsilon B_{z}^{(\varepsilon)} S(z, \zeta)$ for

$$
u(z, \zeta)=\frac{1}{2 \pi} \log |z-\bar{\zeta}|^{2}-\left(\alpha\left(|z-\zeta|^{2}\right)+\frac{1}{2 \pi}\right) \log |z-\zeta|^{2},
$$

and for the function $S(z, \zeta)$ defined by (12.24). By taking (12.6) into account, we can readily verify that $\epsilon B_{z}^{(\varepsilon)} u(z, \zeta)=O(\varepsilon / r) . S(\zeta, z)$ depends on $D$. Since the coefficients of $B^{(\varepsilon)}$ are supported by $F$ however,

$$
\left|B_{z}^{(\varepsilon)} S(\zeta, z)\right| \leq M \mathbb{P}_{\zeta}^{\mathbb{H}}\left(\sigma_{K}<\infty\right) \leq M \mathbb{P}_{\zeta}^{\mathbb{H}}\left(\sigma_{\bigcup_{j=1}^{N} \bar{V}_{j}}<\infty\right), \quad \zeta \in \mathbb{H},
$$

where $M=\sup \left\{\left|B_{z}^{(\varepsilon)} G^{\mathbb{H}}(w, z)\right|: z \in F, w \in K, \varepsilon \in\left(0, \varepsilon_{0}\right), \quad D \in \mathcal{D}_{0}, \widetilde{D} \in \mathcal{D}\right\}$, which is finite by (9.2) and Lemma 12.1. Hence $\varepsilon B_{z}^{(\varepsilon)} S(\zeta, z)=O(\varepsilon / r)$.

The $\ell_{0}$-neighborhood of $F$ will be denoted by $W_{\ell_{0}}$. 
Lemma 12.3. For any $w, \zeta \in D, w \neq \zeta$, it holds that

$$
\begin{aligned}
g(\zeta, w, \varepsilon)= & G(w, \zeta)-P_{0}(w, \zeta)+P_{\varepsilon}(w, \zeta) \\
& +\int_{W_{\ell_{0}}} g(z, w, \varepsilon) A_{\varepsilon, z}\left[G-P_{0}+P_{\varepsilon}\right](z, \zeta) d x_{1} d x_{2} .
\end{aligned}
$$

Proof. According to [10, (15.14)], the self-adjoint elliptic differential operator $A_{\varepsilon}$ admits Green's second formula,

$$
\int_{E}\left(v A_{\varepsilon} u-u A_{\varepsilon} v\right) d x_{i} d x_{2}=\int_{\partial E} \Lambda_{\varepsilon}[u, v] d s,
$$

where $E$ is a bounded domain in $\mathbb{H}$ with smooth boundary $\partial E$ and

$$
\Lambda_{\varepsilon}[u, v]=\sum_{k, \ell=1}^{2} A_{k \ell}^{(\varepsilon)}\left(v \frac{\partial u}{\partial x_{k}} \frac{\partial x_{\ell}}{\partial \mathbf{n}}-u \frac{\partial v}{\partial x_{k}} \frac{\partial x_{\ell}}{\partial \mathbf{n}}\right) .
$$

Here $\mathbf{n}$ is the unit outward normal at $\partial E$.

We fix $w, \zeta \in D, w \neq \zeta$. We then take a large $\ell>0$ such that the rectangle $R_{\ell}=\left\{x_{1}+i x_{2} \in \mathbb{H}:\left|x_{1}\right|<\ell, 0<x_{2}<\ell\right\}$ contains the points $w, \zeta$ and the set $W_{\ell_{0}}$ as well. For each $1 \leq i \leq N$, we choose a smooth Jordan curve $\gamma_{i}$ surrounding $C_{i}$ in such a way that $\gamma_{i} \subset U_{i} \backslash \bar{W}_{\ell_{0}}$ and that its enclosure does not contain $w$ or $\zeta$. We apply the identity (12.16) to

$$
\left\{\begin{array}{l}
E=R_{\ell} \backslash\left(\bigcup_{i=1}^{N} \operatorname{ins} \gamma_{i}\right) \backslash \overline{B_{\delta}(w)} \backslash \overline{B_{\delta}(\zeta)} \\
u(z)=G(z, \zeta)-P_{0}(z, \zeta)+P_{\varepsilon}(z, \zeta) \\
v(z)=g(z, w, \varepsilon)
\end{array}\right.
$$

for a sufficiently small $\delta>0$.

By Lemma 12.1, $A_{\varepsilon} v=0$ on $E$. We can further observe the implication

$$
z \in E \backslash W_{\ell_{0}} \Longrightarrow u(z)=G(z, \zeta), \quad A_{\varepsilon} u(z)=0 .
$$

Indeed, if $\zeta \in F, z \in \mathbb{H} \backslash W_{\ell_{0}}$, then $|z-\zeta|>\ell_{0}$ so that $\alpha\left(|z-\zeta|^{2}\right)=0$ and $u(z)=G(z, \zeta)$. If $\zeta \in \mathbb{H} \backslash F$, then by Lemma 12.1 and 12.4) $a_{i j}^{(\varepsilon)}(\zeta)=2 \delta_{i j}$ so that $P_{\varepsilon}(z, \zeta)=P_{0}(z, \zeta)$ and $u(z)=G(z, \zeta)$. Since $A_{k \ell}^{(\varepsilon)}=\frac{1}{2} \delta_{k \ell}$ on $\mathbb{H} \backslash F$ in view of Lemma 12.1, we obtain from (12.16) and (12.17) the identity

$$
\begin{aligned}
\int_{W_{\ell_{0}}} v(z) A_{\varepsilon} u(z) d x_{1} d x_{2}= & \frac{1}{2} \int_{\Sigma_{\ell}}\left(v \frac{\partial u}{\partial \mathbf{n}}-\frac{\partial v}{\partial \mathbf{n}} u\right) d s+\frac{1}{2} \sum_{i=1}^{N} \int_{\gamma_{i}}\left(v \frac{\partial u}{\partial \mathbf{n}}-\frac{\partial v}{\partial \mathbf{n}} u\right) d s \\
& +\int_{\partial B_{\delta}(w)} \Lambda_{\varepsilon}[u, v] d s+\int_{\partial B_{\delta}(\zeta)} \Lambda_{\varepsilon}[u, v] d s
\end{aligned}
$$

where $\Sigma_{\ell}=\partial R_{\ell} \backslash \partial \mathbb{H}$.

Along $\Sigma_{\ell}, u(z)=G(z, \zeta), v(z)=g(z, w, \varepsilon)$, and both are harmonic and converge to 0 when $|z| \rightarrow \infty$, as they are dominated by $G^{\mathbb{H}}$ of (4.7). Hence we can use Lemma 4.3 to conclude that the first integral on the right-hand side of (12.19) tends to 0 as $\ell \rightarrow \infty$. Along $\gamma_{i}$, we again have $u(z)=G(z, \zeta), v(z)=g(z, w, \varepsilon)$, and both are harmonic and converge to 0 as $z \rightarrow C_{i}, 1 \leq i \leq N$. Therefore, in the same way as in the proof of Theorem 4.5, the second term on the right-hand side of (12.19) can be seen to converge to 0 as we take $\gamma_{i}$ to be the level curve of $u$ shrinking to $C_{i}$. 
Recall the function $\Gamma(z, w)$ defined in (12.5). As for the third term, we can replace $v(z)=g(z, w, \varepsilon)$ by $\widehat{g}(z, w)=-\frac{1}{2 \pi} \log \Gamma(z, w)$ of the same singularity at $w$ to conclude that it tends to $-u(w)=-G(w, \zeta)+P_{0}(w, \zeta)-P_{\varepsilon}(w, \zeta)$ as $\delta \downarrow 0$. As for the fourth term, replacing $u(z)=G(z, \zeta)-P_{0}(z, \zeta)+P_{\varepsilon}(z, \zeta)$ by $\widehat{g}(z, \zeta)$ of the same singularity at $\zeta$, we see that it tends to $v(\zeta)=g(\zeta, w, \varepsilon)$ as $\delta \downarrow 0$.

If we write

$$
\left\{\begin{array}{l}
\widehat{K}_{\varepsilon}(\zeta, z)=A_{\varepsilon, z}\left[G-P_{0}+P_{\varepsilon}\right](z, \zeta), \\
G_{\varepsilon}(\zeta, w)=G(w, \zeta)-P_{0}(w, \zeta)+P_{\varepsilon}(w, \zeta),
\end{array}\right.
$$

then the identity (12.15) is converted into a Fredholm type integral equation:

$$
g(\zeta, w, \varepsilon)=G_{\varepsilon}(\zeta, w)+\int_{W_{\ell_{0}}} \widehat{K}_{\varepsilon}(\zeta, z) g(z, w, \varepsilon) d x_{1} d x_{2} .
$$

In view of (12.4) and (12.7), we have

$$
\left|P_{\varepsilon}(w, \zeta)-P_{0}(w, \zeta)\right| \leq-2 \varepsilon \alpha\left(|w-\zeta|^{2}\right)\left(\left|b_{11}^{(\varepsilon)}(\zeta)\right|+\left|b_{22}^{(\varepsilon)}(\zeta)\right|+\left|b_{12}^{(\varepsilon)}(\zeta)\right|\right)
$$

so that

$$
P_{\varepsilon}(w, \zeta)-P_{0}(w, \zeta)=\varepsilon \eta_{1}^{(\varepsilon)}(w, \zeta), \quad w, \zeta \in \overline{\mathbb{H}},
$$

where $\eta_{1}^{(\varepsilon)}(w, \zeta)$ a continuous function on $\overline{\mathbb{H}} \times \overline{\mathbb{H}}$ bounded uniformly in $\varepsilon \in\left(0, \varepsilon_{0}\right)$, $D \in \mathcal{D}_{0}$ and $\tilde{f}_{\varepsilon}(D)$.

For a function $u(\zeta, w), \zeta, w \in D \cup \partial \mathbb{H}$, we let $\|u\|_{\infty}=\sup _{\zeta, w \in D \cup \partial \mathbb{H}}|u(\zeta, w)|$. If we write $\left(\widehat{K}_{\varepsilon} G_{\varepsilon}\right)(\zeta, w)=\int_{W_{\ell_{0}}} \widehat{K}_{\varepsilon}(\zeta, z) G_{\varepsilon}(z, w) d x_{1} d x_{2}$, then $\left|\left(\widehat{K}_{\varepsilon} G_{\varepsilon}\right)(\zeta, w)\right| \leq$ $\int_{W_{\ell_{0}}}\left|\widehat{K}_{\varepsilon}(\zeta, z)\right|\left(G^{\mathbb{H}}(z, w)+\left|P_{\varepsilon}(z, w)-P_{0}(z, w)\right|\right) d x_{1} d x_{2}$, and we have from Lemma 12.2 and (12.21) that

$\left\|\widehat{K}_{\varepsilon} G_{\varepsilon}\right\|_{\infty} \leq \varepsilon C_{1} \quad$ for $C_{1}$ independent of $\varepsilon \in\left(0, \varepsilon_{0}\right), D \in \mathcal{D}_{0}$ and $\widetilde{f}_{\varepsilon}(D)$.

From Lemma 12.2, we also have, for a constant $C_{2}>0$ independent of $\varepsilon \in\left(0, \varepsilon_{0}\right)$, $D \in \mathcal{D}_{0}$ and $\widetilde{f}_{\varepsilon}(D), \int_{W_{\ell_{0}}}\left|\widehat{K}_{\varepsilon}(\zeta, z)\right| d x_{1} d x_{2} \leq \varepsilon C_{2}$ for any $\zeta \in D \cup \partial \mathbb{H}$. Hence $\left\|\widehat{K}_{\varepsilon}^{(2)} G_{\varepsilon}\right\|_{\infty} \leq \varepsilon^{2} C_{1} C_{2}$ for $\widehat{K}_{\varepsilon}^{(2)} G_{\varepsilon}(\zeta, w)=\widehat{K}_{\varepsilon}\left(\widehat{K}_{\varepsilon} G_{\varepsilon}\right)(\zeta, w)$. Similarly, we have $\left\|\widehat{K}_{\varepsilon}^{(n)} G_{\varepsilon}\right\|_{\infty} \leq \varepsilon^{n} C_{1} C_{2}^{n-1}$ for every $n \geq 1$.

Denote $\varepsilon_{0} \wedge\left(1 /\left(2 C_{2}\right)\right)$ by $\widehat{\varepsilon}_{0}$. For $0<\varepsilon<\widehat{\varepsilon}_{0}$,

$$
\sum_{n=1}^{\infty}\left\|\widehat{K}_{\varepsilon}^{(n)} G_{\varepsilon}\right\|_{\infty} \leq \sum_{n=1}^{\infty} \varepsilon^{n} C_{1} C_{2}^{n-1}<2 \varepsilon C_{1},
$$

and so the convergence

$$
\sum_{n=1}^{\infty} \widehat{K}_{\varepsilon}^{(n)} G_{\varepsilon}(\zeta, w)=\varepsilon \eta_{2}^{(\varepsilon)}(\zeta, w)
$$

is uniform on $D \cup \partial \mathbb{H} \times D \cup \partial \mathbb{H}$, where $\varepsilon \eta_{2}^{(\varepsilon)}(\zeta, w)$ is a continuous function there that is uniformly bounded in $\varepsilon \in\left(0, \widehat{\varepsilon}_{0}\right), D \in \mathcal{D}_{0}$ and $\widetilde{f}_{\varepsilon}(D)$. Moreover, using the bound $g(z, w, \varepsilon) \leq G^{\mathbb{H}}\left(\widetilde{f}_{\varepsilon}(z), \widetilde{f}_{\varepsilon}(w)\right)$, the second term of one can check that the right-hand side of (12.20) is bounded in $\zeta, w \in D$ for any $\varepsilon \in\left(0, \widetilde{\varepsilon}_{0}\right)$ for some $\widetilde{\varepsilon}_{0} \in\left(0, \widehat{\varepsilon}_{0}\right]$.

Solving the equation (12.20) for $g(\zeta, w, \varepsilon)$ and setting $\eta^{(\varepsilon)}(\zeta, w)=\eta_{1}^{(\varepsilon)}(w, \zeta)+$ $\eta_{2}^{(\varepsilon)}(\zeta, w)$, we get the following from (12.21) and (12.22). 
Lemma 12.4. For any $\zeta, w \in D, \zeta \neq w$,

$$
g(\zeta, w, \varepsilon)=G(\zeta, w)+\varepsilon \eta^{(\varepsilon)}(\zeta, w), \quad \varepsilon \in\left(0, \widetilde{\varepsilon}_{0}\right),
$$

where $\eta^{(\varepsilon)}(\zeta, w)$ is a continuous function on $D \cup \partial \mathbb{H} \times D \cup \partial \mathbb{H}$ bounded there uniformly in $\varepsilon \in\left(0, \widetilde{\varepsilon}_{0}\right), D \in \mathcal{D}_{0}$ and $\widetilde{D}=\widetilde{f}_{\varepsilon}(D) \in \mathcal{D}$.

Let us take $\zeta \in \mathbb{H} \backslash F$ in (12.15). By noting that $P_{\varepsilon}(z, \zeta)=P_{0}(z, \zeta)$ and letting $\ell_{0} \downarrow 0$, we arrive at (9.12) as $A_{\varepsilon, z} G(z, \zeta)=\varepsilon B_{z}^{(\varepsilon)} G(z, \zeta), z \in F$. By substituting (12.23) into (9.12), we obtain (9.13). The proof for assertions (iii) and (iv) of Proposition 9.3 is now complete.

We finally prove the assertions (v)-(viii) of Proposition 9.3 .

(v) $G$ can be expressed by the Green function $G^{\mathbb{H}}$ of the ABM $Z^{\mathbb{H}}$ as

$$
G(\zeta, z)=G^{\mathbb{H}}(\zeta, z)-S(\zeta, z), \quad \text { where } \quad S(\zeta, z)=\mathbb{E}_{\zeta}^{\mathbb{H}}\left[G^{\mathbb{H}}\left(Z_{\sigma_{K}}^{\mathbb{H}}, z\right) ; \sigma_{K}<\infty\right] .
$$

By the expression (4.7) of $G^{\mathbb{H}}$ and Proposition 9.3(ii), $B_{z}^{(\varepsilon)} G^{\mathbb{H}}(z, \zeta)_{\zeta_{2}}$ is uniformly bounded on $F \times J$ in $\varepsilon \in\left(0, \varepsilon_{0}\right), D \in \mathcal{D}_{0}$ and $\widetilde{f}_{\varepsilon}(D)$. Furthermore, for any $\varepsilon \in$ $\left(0, \varepsilon_{0}\right), z \in F$ and $\zeta \in \mathbb{H} \backslash \bigcup_{j=1}^{N} V_{j}$,

$$
M=\sup _{\varepsilon \in\left(0, \varepsilon_{0}\right), w \in K, \xi \in F, D \in \mathcal{D}_{0}, \widetilde{D} \in \mathcal{D}}\left|B_{z}^{(\varepsilon)} G^{\mathbb{H}}(w, \xi)\right|<\infty
$$

and

$$
\left|B_{z}^{(\varepsilon)} S(z, \zeta)\right| \leq M \mathbb{P}_{\zeta}^{\mathbb{H}}\left(\sigma_{\bar{U}}<\infty\right),
$$

where $U=\bigcup_{j=1}^{N} U_{j}$. As was observed in the proof of Lemma $5.2($ ii $), \mathbb{P}_{\zeta}^{\mathbb{H}}\left(\sigma_{\bar{U}}<\infty\right)=$ $\int_{\bar{U}} G^{\mathbb{H}}(\zeta, w) \mu(d w)$ for a finite measure $\mu$ concentrated on $\bar{U}$. Since both $B_{z}^{(\varepsilon)} S(z, \zeta)$ and $\mathbb{P}_{\zeta}^{\mathbb{H}}\left(\sigma_{\bar{U}}<\infty\right)$ vanish when $\zeta \in \partial \mathbb{H}$, we deduce from above that

$$
\left|B_{z}^{(\varepsilon)} \frac{\partial}{\partial \zeta_{2}} S(z, \zeta)\right| \leq M \sup _{w \in \bar{U}, \zeta \in J} \frac{\partial}{\partial \zeta_{2}} G^{\mathbb{H}}(\zeta, w) \cdot \mu(\bar{U}), \quad \zeta \in J .
$$

(vi) Let $G^{\mathbb{H}, i}(z, \zeta)$ be the Green function of the ABM on $D^{i}=D \cup C_{i}$ $\left(=\mathbb{H} \backslash\left(K \backslash C_{i}\right)\right)$. Then

$$
G^{\mathbb{H}, i}(z, \zeta)=G^{\mathbb{H}}(z, \zeta)-\mathbb{E}_{\zeta}^{\mathbb{H}}\left[G^{\mathbb{H}}\left(Z_{\sigma_{K \backslash C_{i}}}^{\mathbb{H}}, z\right) ; \sigma_{K \backslash C_{i}}<\infty\right]
$$

According to [5, Corollary 3.4.3], $\varphi^{(i)}$ is the 0-order equilibrium potential of the compact set $C_{i}$ with respect to the transient extended Sobolev space $\left(H_{0, e}^{1}\left(D^{i}\right), \frac{1}{2} \mathbf{D}\right)$, and it admits an expression

$$
\varphi^{(i)}(z)=G^{\mathbb{H}, i} \nu_{i}(z)=\int_{C_{i}} G^{\mathbb{H}, i}(z, \zeta) \nu_{i}(d \zeta)
$$

for some finite positive measure $\nu_{i}$ concentrated on $C_{i}$ in view of the 0 -order version of [5, Lemma 2.3.10] and [9, Exercise 4.2.2]. Hence

$$
\varphi^{(i)}(z)=G^{\mathbb{H}} \nu_{i}(z)-\mathbb{E}_{\nu_{i}}^{\mathbb{H}}\left[G^{\mathbb{H}}\left(Z_{\sigma_{K \backslash C_{i}}}^{\mathbb{H}}, z\right) ; \sigma_{K \backslash C_{i}}<\infty\right] .
$$

Consequently, we have for the same constant $M$ as in the proof of (v),

$$
\left|B_{z}^{(\varepsilon)} \varphi^{(i)}(z)\right| \leq 2 M \nu_{i}\left(C_{i}\right), \quad \varepsilon \in\left(0, \varepsilon_{0}\right), z \in F .
$$


For an open set $G \subset \mathbb{H}$, denote by $\operatorname{Cap}(B ; G)$ the 0 -order capacity of $B \subset G$ relative to $\left(H_{0, e}^{1}(G), \frac{1}{2} \mathbf{D}\right)$. It increases as $B$ increases or $G$ decreases. Moreover, we have $\operatorname{Cap}\left(C_{i}, D^{i}\right)=\nu_{i}\left(C_{i}\right)$ (cf. [9]). Hence (12.26) leads us to a uniform bound

$$
\left|B_{z}^{(\varepsilon)} \varphi^{(i)}(z)\right| \leq 2 M \operatorname{Cap}\left(\bar{U}_{i} ; \mathbb{H} \backslash\left(\bigcup_{k \neq i} \bar{V}_{k}\right)\right), \quad \varepsilon \in\left(0, \varepsilon_{0}\right), \quad z \in F .
$$

(vii) Choose $A_{1}, B_{1}, B_{2}$ and $\ell_{0}$ as in the proof of Lemma 9.6, $F$ is a subset of $\left(A_{1}, B_{1}\right) \times\left(\ell_{0}, B_{2}\right)$. By (4.7), we see that the first integral in (9.14) for $k=1,2$ evaluated for $G^{\mathbb{H}}(\zeta, z)$ in place of $G(\zeta, z)$ is bounded by $\frac{2}{\pi} \frac{\left|x_{1}-\zeta_{1}\right|}{\left(x_{1}-\zeta_{1}\right)^{2}+\ell_{0}^{2}}$ and $\frac{2}{\pi \ell_{0}}$, respectively. The second integral evaluated for $G^{\mathbb{H}}(\zeta, z)$ is bounded by $\frac{4}{\pi \ell_{0}}$ and $\frac{2}{\ell_{0}}$, respectively.

On the other hand, we have for $\zeta \in \mathbb{H}$ and $x_{1} \in \mathbb{R}$ or $x_{2}>0$,

$$
\int_{0}^{\infty}\left|\frac{\partial}{\partial x_{1}} G^{\mathbb{H}}\left(\zeta, x_{1}+i x_{2}\right)\right| d x_{2} \leq 1, \quad \int_{-\infty}^{\infty}\left|\frac{\partial}{\partial x_{2}} G^{\mathbb{H}}\left(\zeta, x_{1}+i x_{2}\right)\right| d x_{1} \leq 2 .
$$

Since $|z-\zeta|>b$ for $(z, \zeta) \in F \times K$, we have for any $\zeta \in K$,

$$
\begin{gathered}
\int_{-\infty}^{\infty} \mathbf{1}_{F}(z)\left|\frac{\partial}{\partial x_{1}} G^{\mathbb{H}}(\zeta, z)\right| d x_{1} \leq \frac{2}{\pi b^{2}}\left(B_{1}-A_{1}\right)^{2}, \\
\int_{0}^{\infty} \mathbf{1}_{F}(z)\left|\frac{\partial}{\partial x_{2}} G^{\mathbb{H}}(\zeta, z)\right| d x_{2} \leq \frac{2}{\pi b^{2}} B_{2}^{2} .
\end{gathered}
$$

Denote by $c$ the maximum of the constants in (12.29) and 2. Then each of the four integrals in (9.14) evaluated for the function $S$ in (12.24) admits a bound $c \frac{\partial}{\partial \zeta_{2}} \mathbb{P}_{\zeta}^{\mathbb{H}}\left(\sigma_{\bar{U}}<\infty\right)$, which is in turn dominated by

$$
c \sup _{w \in \bar{U}, \zeta \in J} \frac{\partial}{\partial \zeta_{2}} G^{\mathbb{H}}(\zeta, w) \mu(\bar{U}),
$$

as in the proof of $(\mathbf{v})$ above.

(viii) By virtue of (12.25), (12.28) and (12.29), we see that each of the four integrals in (9.15) is dominated by

$$
2 c \nu_{j}\left(C_{j}\right) \leq 2 c \operatorname{Cap}\left(\bar{U}_{j} ; \mathbb{H} \backslash\left(\bigcup_{k \neq j} \bar{V}_{k}\right)\right) \text {. }
$$

\section{ACKNOWLEDGEMENT}

The authors would like to thank R. Bauer for clarifying some details of some of the proofs in [2] and [3].

\section{REFERENCES}

[1] Lars V. Ahlfors, Complex analysis. An introduction to the theory of analytic functions of one complex variable; International Series in Pure and Applied Mathematics, 3rd ed., McGrawHill Book Co., New York, 1978. MR510197 (80c:30001)

[2] Robert O. Bauer and Roland M. Friedrich, On radial stochastic Loewner evolution in multiply connected domains, J. Funct. Anal. 237 (2006), no. 2, 565-588, DOI 10.1016/j.jfa.2005.12.023. MR2230350(2007d:60007) 
[3] Robert O. Bauer and Roland M. Friedrich, On chordal and bilateral SLE in multiply connected domains, Math. Z. 258 (2008), no. 2, 241-265, DOI 10.1007/s00209-006-0041-z. MR2357634 (2009b:60292)

[4] Z.-Q. Chen, Brownian Motion with Darning. Lecture notes for talks given at RIMS, Kyoto University. 2012.

[5] Zhen-Qing Chen and Masatoshi Fukushima, Symmetric Markov processes, time change, and boundary theory, London Mathematical Society Monographs Series, vol. 35, Princeton University Press, Princeton, NJ, 2012. MR2849840 (2012m:60176)

[6] John B. Conway, Functions of one complex variable. II, Graduate Texts in Mathematics, vol. 159, Springer-Verlag, New York, 1995. MR1344449 (96i:30001)

[7] Shawn Drenning, Excursion reflected Brownian motion and loewner equations in multiply connected domains, ProQuest LLC, Ann Arbor, MI, 2011. Thesis (Ph.D.)-The University of Chicago. MR2992562

[8] Peter L. Duren, Univalent functions, Grundlehren der Mathematischen Wissenschaften [Fundamental Principles of Mathematical Sciences], vol. 259, Springer-Verlag, New York, 1983. MR708494 (85j:30034)

[9] Masatoshi Fukushima, Yōichi Ōshima, and Masayoshi Takeda, Dirichlet forms and symmetric Markov processes, de Gruyter Studies in Mathematics, vol. 19, Walter de Gruyter \& Co., Berlin, 1994. MR1303354 (96f:60126)

[10] P. R. Garabedian, Partial Differential Equations, AMS Chelsia, 2007, republication of 1964 edition

[11] John B. Garnett and Donald E. Marshall, Harmonic measure, New Mathematical Monographs, vol. 2, Cambridge University Press, Cambridge, 2005. MR2150803 (2006g:31002)

[12] Yûsaku Komatu, On conformal slit mapping of multiply-connected domains, Proc. Japan Acad. 26 (1950), no. 7, 26-31. MR0046437 (13,734c)

[13] Gregory F. Lawler, Conformally invariant processes in the plane, Mathematical Surveys and Monographs, vol. 114, American Mathematical Society, Providence, RI, 2005. MR2129588 (2006i:60003)

[14] Gregory F. Lawler, The Laplacian-b random walk and the Schramm-Loewner evolution, Illinois J. Math. 50 (2006), no. 1-4, 701-746 (electronic). MR2247843 (2007k:60261)

[15] John W. Milnor, Topology from the differentiable viewpoint. Based on notes by David W. Weaver, The University Press of Virginia, Charlottesville, Va., 1965. MR0226651 (37 \#2239)

[16] Zeev Nehari, Conformal mapping, Dover Publications, Inc., New York, 1975. Reprinting of the 1952 edition. MR0377031 (51 \#13206)

[17] Sidney C. Port and Charles J. Stone, Brownian motion and classical potential theory. Probability and Mathematical Statistics, Academic Press [Harcourt Brace Jovanovich, Publishers], New York-London, 1978. MR0492329 (58 \#11459)

[18] M. Tsuji, Potential theory in modern function theory, Maruzen Co., Ltd., Tokyo, 1959. MR $0114894(22 \# 5712)$

Department of Mathematics, University of Washington, Seattle, Washington 98195 E-mail address: zqchen@uw.edu

Branch of Mathematical Science, Osaka University, Toyonaka, Osaka 560-0043, JAPAN

E-mail address: fuku2@mx5.canvas.ne.jp

Department of Mathematics, University of Washington, Seattle, Washington 98195

E-mail address: rohde@math.washington.edu 